\title{
Flat Extensions of Positive Moment Matrices: Recursively Generated Relations
}

\author{
Raúl E. Curto \\ Lawrence A. Fialkow
}

Author address:

Department of Mathematics, The University of Iowa, Iowa City, IOWA 52242

E-mail address: curto@math.uiowa.edu

Department of Mathematics and Computer Science, State UniverSity OF New YoRk, New PALtz, NY 12561

E-mail address: fialkow@mcs.newpaltz.edu 
1991 Mathematics Subject Classification. Primary 47A57/44A60, 30E05; Secondary 15A57, 15-04, 47N40.

Research partially supported by grants from the National Science Foundation.

ABstract. We develop new computational tests for existence and uniqueness of representing measures $\mu$ in the Truncated Complex Moment Problem:

$$
\gamma_{i j}=\int \bar{z}^{i} z^{j} d \mu \quad(0 \leq i+j \leq 2 n) .
$$

We characterize the existence of finitely atomic representing measures in terms of positivity and extension properties of the moment matrix $M(n)(\gamma)$ associated with $\gamma \equiv \gamma^{(2 n)}: \gamma_{00}, \ldots, \gamma_{0,2 n}, \ldots, \gamma_{2 n, 0}, \gamma_{00}>0$ (Theorem 1.5). We study conditions for flat (i.e., rank-preserving) extensions $M(n+1)$ of $M(n) \geq 0$; each such extension corresponds to a distinct rank $M(n)$-atomic representing measure, and each such measure is minimal among representing measures in terms of the cardinality of its support. For a natural class of moment matrices satisfying the tests of recursive generation, recursive consistency, and normal consistency, we reduce the existence problem for minimal representing measures to the solubility of small systems of multivariable algebraic equations (Theorem 2.7). In a variety of applications, including cases of the quartic moment problem $(n=2$; Theorem 1.10), we apply these tests so as to construct flat extensions and minimal representing measures. In other examples, we use these tests to demonstrate the non-existence of representing measures or the non-existence of minimal representing measures.

Key words and phrases. Truncated complex moment problem, moment matrix extension block, flat extensions of positive matrices, recursively generated relations, algebraic variety of a moment sequence. 


\section{Contents}

$\begin{array}{ll}\text { Chapter 1. Introduction } & 1\end{array}$

$\begin{array}{lll}\text { Chapter 2. Flat Extensions for Moment Matrices } & 11\end{array}$

Chapter 3. The Singular Quartic Moment Problem 25

Chapter 4. The Algebraic Variety of $\gamma \quad 32$

Chapter 5. $\quad$ J.E. McCarthy's Phenomenon and the Proof of Theorem 1.544

Summary of Results $\quad 49$

Bibliography $\quad 52$

List of Symbols $\quad 55$ 
CONTENTS 
For Inés, Carina, Roxanna and Vilsa

and

For Deborah, Velaho, Ruth, Jarmel, Tawana and Atiba 



\section{CHAPTER 1}

\section{Introduction}

Consider a collection of complex numbers $\gamma \equiv \gamma^{(2 n)}: \gamma_{00}, \gamma_{01}, \gamma_{10}, \ldots, \gamma_{0,2 n}$, $\gamma_{1,2 n-1}, \ldots, \gamma_{2 n-1,1}, \gamma_{2 n, 0}$, with $\gamma_{00}>0$ and $\gamma_{j i}=\overline{\gamma_{i j}}$. The truncated complex moment problem (TCMP) entails finding a positive Borel measure supported in the complex plane $\mathbb{C}$ such that $\gamma_{i j}=\int \bar{z}^{i} z^{j} d \mu(0 \leq i+j \leq 2 n) ; \gamma$ is called a truncated moment sequence (of order $2 n$ ) and $\mu$ is called a representing measure for $\gamma$.

In the present paper we provide new necessary or sufficient conditions for the existence of representing measures, particularly those which are minimal in the sense of being finitely atomic with the fewest atoms possible. In a variety of examples, including the so-called quartic moment problem (Theorem 1.10), we explicitly construct minimal representing measures, or we establish the existence of minimal representing measures via the theory of flat extensions of moment matrices (Theorem 2.7). We also identify new computational tests that can be used to prove the non-existence of representing measures, or the non-existence of finitely atomic representing measures. These tests are used to illustrate, in very concrete terms, new phenomena, associated with higher-dimensional moment problems, that do not appear in the classical one-dimensional moment problem. We exhibit the failure of recursive consistency in Example 2.2, and we demonstrate the absence of normal consistency in Example 2.5; either phenomenon implies the non-existence of finitely atomic representing measures. In Examples 4.4-4.7 we illustrate the variety obstruction to the existence of any representing measure. All of our results (both positive and negative) indicate very explicitly why multivariable moment problems are so intractable; even in the positive cases, the existence of minimal measures for TCMP reduces to the solubility of small systems of multivariable algebraic equations, systems for which there is at present no definitive theory.

TCMP is closely related to several other moment problems: the full complex moment problem prescribes moments of all orders, i.e., $\gamma=\left(\gamma_{i j}\right)_{i, j=0}^{\infty}([\mathbf{A k h}]$, $[\mathbf{S h T}]$ ); the $K$-moment problem (truncated or full) prescribes a closed set $K \subseteq \mathbb{C}$ which is to contain the support of the representing measure ([AK], $[\mathbf{A k h}],[\mathbf{A t z}]$, $[\mathbf{H a u}],[\mathbf{K r N}],[\mathbf{P u 1}],[\mathbf{S c h} 2],[\mathbf{S h T}])$; and the multidimensional moment problem extends each of these problems to measures supported in $\mathbb{C}^{k}([\mathbf{B e r}],[\mathbf{B C J}]$, [Hav1], [Hav2], [Sch1], [Fug], [McG], [StSz4]). Moreover, the $k$-dimensional complex moment problem is equivalent to the $2 k$-dimensional real moment problem $[\mathbf{C u F 4}$, Section 6]. All of the above mentioned problems generalize classical power moment problems on the real line, whose study was initiated by Stieltjes, Riesz, Hamburger, and Hausdorff ([AK], [A khh], [Hau], [KrN], [Lan], [Rez1], $[\mathbf{S a r}],[\mathbf{S h T}])$. Recently, in response to our question, J. Stochel [Sto] proved that a solution to the multidimensional truncated $K$-moment problem actually implies a solution to the corresponding full $K$-moment problem. TCMP is also related to subnormal operator theory, the study of unbounded subnormal operators, polynomial 
hyponormality and joint hyponormality (see $[\mathbf{C u 1}],[\mathbf{C u 2}],[\mathbf{C u} 3],[\mathbf{C u F 1}],[\mathbf{C u F 2}]$, $[$ CuP1], [CuP2], [JeL], [Li], [McCY], [Pu1], [Pu3], [Pu4], [Pu5], [Pu6], [Sar], $[\mathrm{SeS}],[$ StSz1], [StSz2], [StSz3], [StSz4], [Sza1], [Sza2]).

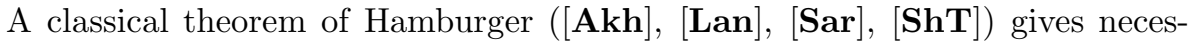
sary and sufficient conditions for the solution of the full moment problem on the line, that is, $K=\mathbb{R}$ : a real sequence $\beta \equiv\left(\beta_{i}\right)_{i=0}^{\infty}$ with $\beta_{0}>0$ has a representing measure supported in $\mathbb{R}$ if and only if for each $k \geq 0$, the Hankel matrix $H(k):=\left(\beta_{i+j}\right)_{0 \leq i+j \leq 2 k}$ is positive semi-definite. Hamburger's Theorem serves as a prototype for much of moment theory because it provides a concrete criterion closely related to the moments; nevertheless, when $K=\mathbb{R}^{n}(n>1)$, the most direct analogue of Hamburger's Theorem is false ([BCJ], $[\mathbf{S c h} \mathbf{1}])$, and the $K=\mathbb{R}^{n}$ full moment problem (including the full complex moment problem for $K=\mathbb{C}$ ) remains unsolved (cf. [Atz], [Ber], [BeM], [Cas], [CuP2], [Fug], [Pu3], [Sch2]).

In a different direction, M. Riesz ([ShT], $[\mathbf{A k h}]$ ) proved that $\beta$ (as above) has a representing measure supported in a closed set $K \subseteq \mathbb{R}$ if and only if, whenever a polynomial $a_{0}+a_{1} t+\cdots+a_{n} t^{n}$ (with complex coefficients) is nonnegative on $K$, then $a_{0} \beta_{0}+a_{1} \beta_{1}+\cdots+a_{n} \beta_{n} \geq 0$. Haviland ([Hav1], [Hav2]) subsequently extended this result to the multivariable full $K$-moment problem. Although Riesz's Theorem solves the full moment problem in principle, it is very difficult to verify the Riesz criterion for a particular sequence $\beta$.

In the sequel we are concerned with concrete tests for existence and uniqueness in TCMP. In ([CuF4], [CuF5], [Fia1]) we initiated an approach to TCMP based on positivity and extension properties of the moment matrix $M(n) \equiv M(n)(\gamma)$ associated with a truncated moment sequence $\gamma$ (see below for terminology and notation). If $\mu$ is any representing measure for $\gamma$, then card supp $\mu \geq \operatorname{rank} M(n)$ (cf. (1.5) below); the main result of [CuF4] characterizes the existence of representing measures $\mu$ which are minimal in the sense that $\operatorname{card} \operatorname{supp} \mu=\operatorname{rank} M(n)$.

TheOREM 1.1. [CuF4, Theorem 5.13] $\gamma$ has a rank $M(n)$-atomic representing measure if and only if $M(n) \geq 0$ and $M(n)$ admits a flat extension $M(n+1)$, i.e., $M(n)$ can be extended to a positive moment matrix $M(n+1)$ satisfying $\operatorname{rank} M(n+1)=\operatorname{rank} M(n)$.

In view of Theorem 1.1, a minimal representing measure for $\gamma^{(2 n)}$ corresponds to a solution of the following system of $(n+1)(2 n+1)$ polynomial equations in the $2 \cdot \operatorname{rank} M(n)$ unknowns $a_{k}>0, z_{k} \in \mathbb{C}(1 \leq k \leq r \equiv \operatorname{rank} M(n))$ :

$$
\gamma_{i j}=\sum_{k=1}^{r} a_{k} \bar{z}_{k}^{i} z_{k}^{j} \quad(0 \leq i+j \leq 2 n)
$$

In the sequel we show how to reduce the existence problem for minimal representing measures to solubility of much smaller systems. For example, consider the quartic moment problem $(n=2)$ with $\operatorname{rank} M(2)=5$ and with the fifth column of $M(2)$ dependent on the first three columns. In this case, the above system consists of 15 equations in 10 unknowns, but in Theorem 1.10(iv) we reduce the flat extension problem for this $M(2)$ to solubility of a single equation of the form

$$
\alpha+\beta z+\gamma \bar{z}+\delta|z|^{2}=0
$$

where $\alpha, \beta, \gamma, \delta$ are computable algebraic expressions in the moments $\gamma_{i j}(0 \leq i+j \leq$ 2). 
The present work is primarily devoted to providing computational criteria for the existence of flat moment matrix extensions. Before we discuss our results in detail, it is useful to recall the truncated Hamburger moment problem (THMP), the truncated moment problem for one real variable. Let $\beta \equiv \beta^{(2 n)}: \beta_{0}, \ldots, \beta_{2 n}$ denote a collection of real numbers with $\beta_{0}>0$, and let $H(n) \equiv H(n)(\beta)$ denote the Hankel matrix $\left(\beta_{i+j}\right)_{0 \leq i+j \leq 2 n}$. For $0 \leq j \leq n$, let $T^{j}:=\left(\beta_{j}, \ldots, \beta_{j+n}\right)^{T}$ denote the $j$-th column of $H(n)$ (and set $1=T^{0}$ ). In case $H(n)$ is singular, we set $r:=\min \left\{j: T^{j} \in\left\langle 1, \ldots, T^{j-1}\right\rangle\right\}$; then $1 \leq r \leq n$ and there exist unique real scalars $a_{0}, \ldots, a_{r-1}$ such that $T^{r}=a_{0} 1+\cdots+a_{r-1} T^{r-1}$. We say that $H(n)$ is recursively generated if it is nonsingular, or if it is singular (as above) and

$$
T^{r+s}=a_{0} T^{s}+\cdots+a_{r-1} T^{r+s-1} \quad(0 \leq s \leq n-r) .
$$

(We remark that the structure theorem for positive Hankel matrices [CuF3, Theorem 2.4] shows that a positive singular Hankel matrix always satisfies the identity in (1.1) for $0 \leq s \leq n-r-1$, so a positive singular $H(n)$ is recursively generated if and only if $T^{n}=a_{0} T^{n-r}+\cdots+a_{r-1} T^{n-1}$.)

The following results of $[\mathbf{C u F 3}]$ characterize the existence and uniqueness of representing measures for $\beta$ and provide a procedure for explicitly constructing representing measures.

Theorem 1.2. (THMP-Existence; Even Case) [CuF3, Theorem 3.9] The following statements are equivalent.

(i) There exists a positive Borel measure $\mu$, with $\operatorname{supp} \mu \subseteq \mathbb{R}$, such that $\beta_{j}=$ $\int t^{j} d \mu(0 \leq j \leq 2 n)$

(ii) $\beta$ has a finitely atomic representing measure;

(iii) $\beta$ has a rank $H(n)$-atomic representing measure;

(iv) $H(n)$ admits a positive extension $H(n+1)$;

(v) $H(n) \geq 0$ and $H(n)$ admits a flat (i.e., rank-preserving) extension $H(n+1)$;

(vi) $H(n) \geq 0$ and $H(n)$ is recursively generated.

(Note that (vi) in Theorem 1.2 is a concrete condition that can be checked just by using elementary linear algebra.)

Theorem 1.3. (THMP-Uniqueness; Even Case) [CuF3, Theorem 3.10] Suppose $\beta \equiv \beta^{(2 n)}$ admits a representing measure.

(i) If $H(n)$ is singular, let $p(t):=t^{r}-\left(a_{0}+\cdots+a_{r-1} t^{r-1}\right)$; then $p$ has $r$ distinct real roots $t_{0}, \ldots, t_{r-1}$, and the unique representing measure for $\beta$ is of the form

$$
\mu=\sum_{i=0}^{r-1} \rho_{i} \delta_{t_{i}},
$$

where $\delta_{t_{i}}$ is the atomic measure with support $\left\{t_{i}\right\}$, and the densities $\rho_{0}, \ldots$, $\rho_{r-1}$ are uniquely determined by the Vandermonde equation

$$
V\left(t_{0}, \ldots, t_{r-1}\right)\left(\rho_{0}, \ldots, \rho_{r-1}\right)^{T}=\left(\beta_{0}, \ldots, \beta_{r-1}\right)^{T} .
$$

(ii) If $H(n)$ is nonsingular, then to each choice of $\beta_{2 n+1} \in \mathbb{R}$ there exists a unique flat extension $H(n+1)\left[\beta_{2 n+1}\right]$ which is positive and recursively generated; thus, the corresponding moment problem for $H(n+1)\left[\beta_{2 n+1}\right]$ has a 
unique representing measure (as described above), which is an $(n+1)$-atomic representing measure for $\beta$.

In $[\mathbf{C u F 4}]$ we introduced the moment matrix $M(n)(\gamma)$ in order to study the extent to which TCMP admits analogues of Theorems 1.2 and 1.3 (with $\gamma$ replacing $\beta$ and $M(n)(\gamma)$ replacing $H(n)(\beta))$. Given $n \geq 1$, let $m \equiv m(n):=(n+1)(n+2) / 2$. We introduce the following lexicographic order on the rows and columns of complex matrices of size $m: 1, Z, \bar{Z}, Z^{2}, \bar{Z} Z, \bar{Z}^{2}, \ldots, Z^{n}, \ldots, \bar{Z}^{n}$; rows or columns indexed by $1, Z, \ldots, Z^{n}$ are said to be analytic. Let $\gamma: \gamma_{00}, \ldots, \gamma_{0,2 n}, \ldots, \gamma_{2 n, 0}$ be a truncated moment sequence, and for $0 \leq i, j \leq n$ define the $(i+1) \times(j+1)$ matrix $M[i, j]$ whose entries are the moments of order $i+j$ :

$$
M[i, j]:=\left(\begin{array}{cccc}
\gamma_{i, j} & \gamma_{i+1, j-1} & \cdots & \gamma_{i+j, 0} \\
\gamma_{i-1, j+1} & \gamma_{i, j} & \cdots & \gamma_{i+j-1,1} \\
\vdots & \vdots & \ddots & \vdots \\
\gamma_{0, i+j} & \gamma_{1, i+j-1} & \cdots & \gamma_{j, i}
\end{array}\right)
$$

We now define the moment matrix $M(n) \equiv M(n)(\gamma)$ via the block decomposition $M(n):=(M[i, j])_{0 \leq i, j \leq n}$. For example, if $n=1$, the quadratic moment problem for $\gamma \equiv \gamma^{(2)}: \gamma_{00}, \gamma_{01}, \gamma_{10}, \gamma_{02}, \gamma_{11}, \gamma_{20}$ corresponds to

$$
M(1)=\left(\begin{array}{ll}
M[0,0] & M[0,1] \\
M[1,0] & M[1,1]
\end{array}\right)=\left(\begin{array}{lll}
\gamma_{00} & \gamma_{01} & \gamma_{10} \\
\gamma_{10} & \gamma_{11} & \gamma_{20} \\
\gamma_{01} & \gamma_{02} & \gamma_{11}
\end{array}\right)
$$

Let $\mathcal{P}_{n} \subseteq \mathbb{C}[z, \bar{z}]$ denote the polynomials in $z$ and $\bar{z}$ of total degree at most $n$. For $p \in \mathcal{P}_{n}, p(z, \bar{z}) \equiv \sum_{0 \leq i+j \leq n} a_{i j} \bar{z}^{i} z^{j}$, let $\hat{p}:=\left(a_{00}, a_{01}, a_{10}, \ldots, a_{0 n}, \ldots, a_{n 0}\right)^{T} \in$ $\mathbb{C}^{m(n)}$. For $M \in M_{m(n)}(\mathbb{C})$ and $p, q \in \mathcal{P}_{n}$, let $\langle p, q\rangle_{M}:=(M \hat{p}, \hat{q})$. The basic connection between the moment matrix $M(n)$ and any representing measure $\mu$ is provided by the identity $\int p \bar{q} d \mu=\langle p, q\rangle_{M(n)}\left(p, q \in \mathcal{P}_{n}\right)[\mathbf{C u F} 4]$; in particular, $(M(n) \hat{p}, \hat{p})=\int|p|^{2} d \mu \geq 0$, so if $\gamma$ admits a representing measure, then $M(n) \geq 0$.

The main example of $[\mathbf{C u F 4}]$ concerns a close analogue of Theorems 1.2-1.3 in case $M(n) \geq 0$ and $\gamma$ is of flat data type, i.e., $\operatorname{rank} M(n)=\operatorname{rank} M(n-1)$.

THEOREM 1.4 .

(i) $[\mathbf{C u F 4}$, Theorem 4.7] Let $M \equiv M(\infty)$ be a finite-rank positive infinite moment matrix (of the form $(M[i, j])_{i, j=0}^{\infty}$ ), and let $r:=\operatorname{rank} M$. Then $M$ has a unique representing measure $\mu$, which is $r$-atomic, and there exist unique scalars $a_{0}, \ldots, a_{r-1}$ such that $Z^{r}=a_{0} 1+a_{1} Z+\cdots+a_{r-1} Z^{r-1}$ in $\mathcal{C}_{M}$, the column space of $M$. If $p(z):=z^{r}-\left(a_{0}+\cdots+a_{r-1} z^{r-1}\right)$, then $p$ has $r$ distinct roots, $z_{0}, \ldots, z_{r-1}$, and $\mu=\sum_{i=0}^{r-1} \rho_{i} \delta_{z_{i}}$, where

$$
V\left(z_{0}, \ldots, z_{r-1}\right)\left(\rho_{0}, \ldots, \rho_{r-1}\right)^{T}=\left(\gamma_{0,0}, \ldots, \gamma_{0, r-1}\right)^{T}
$$

(ii) $[\mathbf{C u F 4}$, Theorem 5.4 and Corollary 5.12] If $\gamma$ is of flat data type and $M(n) \equiv$ $M(n)(\gamma) \geq 0$, then $M(n)$ admits a unique flat extension $M(n+1)$. Thus, $M(n)$ also admits a unique flat extension $M(\infty) \geq 0$. In this case, $\gamma$ admits a unique representing measure, as described in (i). (If rank $M(n)>2 n+1$, the auxiliary moments $\gamma_{0,2 n+1}, \ldots, \gamma_{0, r-1}$ needed in (1.3) are located in the flat extension $M(r)$.) 
Despite Theorem 1.4, there are significant differences between TCMP and THMP. For the quadratic moment problem, positivity of $M(1)$ implies the existence of $\operatorname{rank} M(1)$-atomic representing measures [CuF4, Theorem 6.1]; however, for the singular case with $\operatorname{rank} M(1)=2$, we proved in $[\mathbf{C u F 4}$, Theorem 6.1] that $\gamma^{(2)}$ has infinitely many 2-atomic representing measures, so there is no close analogue of Theorem 1.3(i) for uniqueness in TCMP.

A more striking distinction between TCMP and THMP concerns the role of flat extensions. Recently, J.E. McCarthy $[\mathbf{M c C}]$ proved that there exist truncated moment sequences $\gamma$ admitting representing measures, but having no rank $M(n)(\gamma)$ atomic representing measures (see Chapter 5 below); thus, by Theorem 1.1, for these sequences $\gamma, M(n)(\gamma)$ admits no flat extension $M(n+1)$. McCarthy's result shows that TCMP has no close analogue of Theorem 1.2(i) $\Leftrightarrow(\mathrm{v})$; however, the following result provides a TCMP partial analogue of Theorem $1.2(\mathrm{i}) \Leftrightarrow(\mathrm{ii})$.

THEOREM 1.5. For $\gamma \equiv \gamma^{(2 n)}$, the following statements are equivalent.

(i) $\gamma$ has a representing measure with moments up to order $2(n+1)$;

(ii) $\gamma$ has a representing measure with moments of all orders;

(iii) $\gamma$ has a compactly supported representing measure;

(iv) $\gamma$ has a finitely-atomic representing measure (in this case, there is some representing measure with at most $(n+2)(2 n+3)$ atoms $)$;

(v) $M(n)(\gamma) \geq 0$ and for some $k \geq 0, M(n)$ admits a positive extension $M(n+k)$ which in turn admits a flat extension $M(n+k+1)$ (in this case, we can take $\left.k \leq 2 n^{2}+6 n+6\right)$

(vi) $M(n)(\gamma) \geq 0$ and the semi-inner product $\langle\cdot, \cdot\rangle_{M(n)(\gamma)}$ induced by $M(n)(\gamma)$ on $\mathcal{P}_{n}$ admits an isometric extension to a semi-inner product on $\mathbb{C}[z, \bar{z}]$ compatible with the structure of a finite-rank infinite moment matrix.

Theorem 1.5 is proved in Chapter 5, and it evolved from a Numerical Methods course taught by the second-named author in Fall 1994; during this course we recognized that a classical theorem of multivariable quadrature due to V. Tchakaloff [Tch] could be adapted to prove (iii) $\Rightarrow$ (iv). After informally communicating this result to several colleagues, we learned from J. McCarthy of an alternate proof of (iii) $\Rightarrow$ (iv) that uses convexity theory. Subsequently, in response to our question, M. Putinar $[\mathbf{P u} 7]$ generalized Tchakaloff's Theorem so as to prove (i) $\Rightarrow($ iv).

The preceding discussion makes clear the distinction between TCMP and THMP with respect to uniqueness and flat extensions. Another important distinction can be found in the role played by positivity and recursiveness. For THMP, the existence of representing measures is equivalent to the condition $H(n) \geq 0$ and $H(n)$ recursively generated (Theorem $1.2(\mathrm{i}) \Leftrightarrow(\mathrm{vi}))$. The analogue of this result for TCMP is decidedly false, as we next explain.

For $M \in M_{m(n)}(\mathbb{C})$, let $\mathcal{C}_{M}$ denote the column space of $M$, i.e., $\mathcal{C}_{M}:=$ $\left\langle 1, Z, \bar{Z}, \ldots, Z^{n}, \ldots, \bar{Z}^{n}\right\rangle \subseteq \mathbb{C}^{m(n)}$. For $p \in \mathcal{P}_{n}, p(z, \bar{z}) \equiv \sum_{i, j} a_{i j} \bar{z}^{i} z^{j}$, we define $p(Z, \bar{Z}):=\sum_{i, j} a_{i j} \bar{Z}^{i} Z^{j} \in \mathcal{C}_{M}$; thus, for $p, q \in \mathcal{P}_{n}$, we have $M \hat{p}=p(Z, \bar{Z})$ and $\langle p, q\rangle_{M}=(M \hat{p}, \hat{q})=(p(Z, \bar{Z}), \hat{q})$. We recall from [CuF4, Theorem 2.1] that $M \in M_{m(n)}(\mathbb{C})$ is of the form $M(n)(\gamma)$ for some truncated moment sequence $\gamma$ if and only if $M=M^{*},\langle 1,1\rangle_{M}>0,\langle f, g\rangle_{M}=\langle\bar{g}, \bar{f}\rangle_{M}\left(f, g \in \mathcal{P}_{n}\right)$ (symmetric property), $\langle z f, g\rangle_{M}=\langle f, \bar{z} g\rangle_{M}\left(f, g \in \mathcal{P}_{n-1}\right)$, and $\langle z f, z g\rangle_{M}=\langle\bar{z} f, \bar{z} g\rangle_{M}\left(f, g \in \mathcal{P}_{n-1}\right)$ (normality). The following Structure Theorem for positive moment matrices (based 
on $[\mathbf{C u F 4}$, Theorem 3.14]) provides the foundation for our analysis of positive extensions and representing measures; the second part of the statement does not appear in [CuF4, Theorem 3.14], but is implicit in its proof.

Theorem 1.6. Assume $M(n)(\gamma) \geq 0$. If $p, q, p q \in \mathcal{P}_{n-1}$ and $p(Z, \bar{Z})=\mathbf{0}$, then $(p q)(Z, \bar{Z})=\mathbf{0}$; moreover, if $p, q, p q \in \mathcal{P}_{n}$ and $p(Z, \bar{Z})=\mathbf{0}$, then $[p q(Z, \bar{Z})]_{(n-1)}=$ 0 .

(Here, and in the sequel, $[\mathbf{w}]_{(d)}$ denotes the truncation of the vector $\mathbf{w} \in \mathbb{C}^{m(n)}$ through entries of degree at most $d$.)

By analogy with Hankel matrices (cf. (1.1) and the subsequent remark), we say that $M(n)$ is recursively generated if it satisfies

(RG)

$$
p, q, p q \in \mathcal{P}_{n}, p(Z, \bar{Z})=\mathbf{0} \Longrightarrow(p q)(Z, \bar{Z})=\mathbf{0} .
$$

It follows readily from Theorem 1.6 and the Extension Principle [Fia1] (cf. Chapter 2) that if $M(n)$ admits a positive extension $M(n+1)$, then $M(n)$ is recursively generated; moreover, (1.4) below implies that if $\gamma$ has a representing measure, then $M(n)$ is recursively generated. In [CuF5], we used a difficult construction of K. Schmüdgen [Sch1] to exhibit a positive invertible, hence recursively generated, $M(3)$ having no representing measure. Since a positive invertible moment matrix always has a positive invertible extension $M(n+1)$, it follows from this example that TCMP does not admit an analogue of Theorem 1.2(i) $\Leftrightarrow$ (iv). In the sequel we exhibit several new (and hopefully more transparent) examples of positive recursively generated moment matrices which do not have representing measures. We do this by identifying new obstructions to the existence of representing measures or positive extensions; the first of these has an algebraic-geometric flavor.

Recall from [CuF4, Proposition 3.1] that if $\mu$ is a representing measure for $\gamma$, then

$$
\text { For } p \in \mathcal{P}_{n}, p(Z, \bar{Z})=\mathbf{0} \Longleftrightarrow \operatorname{supp} \mu \subseteq \mathcal{Z}(p):=\{z \in \mathbb{C}: p(z, \bar{z})=0\} .
$$

It follows from [CuF4, Corollary 3.5] that

$$
\text { If } \mu \text { is a representing measure for } \gamma \text {, then } \operatorname{card} \operatorname{supp} \mu \geq \operatorname{rank} M(n) \text {. }
$$

Motivated by (1.4), we now introduce the variety of $\gamma$, defined by

$$
\mathcal{V}(\gamma):=\bigcap_{\substack{p \in \mathcal{P}_{n} \\ p(Z, \bar{Z})=\mathbf{0}}} \mathcal{Z}(p) .
$$

$\mathcal{V}(\gamma)$ is a closed (possibly empty) subset of the plane, and (1.4)-(1.5) imply

If $\mu$ is a representing measure for $\gamma$, then $\operatorname{supp} \mu \subseteq \mathcal{V}(\gamma)$, and $\operatorname{rank} M(n) \leq \operatorname{card} \operatorname{supp} \mu \leq \operatorname{card} \mathcal{V}(\gamma)$.

Let $\rho(\gamma):=\operatorname{card} \mathcal{V}(\gamma)-\operatorname{rank} M(n)(\gamma)$; from (1.7) it is clear that the condition $\rho(\gamma)<0$ is an obstruction to the existence of representing measures. In Chapter 4 we provide several examples which illustrate $M(n)(\gamma)$ positive and recursively generated, but $\rho(\gamma)<0$ (e.g., Examples 4.4 and 4.6).

We consider next the case when $M(n)(\gamma)$ is positive, recursively generated, and $\rho(\gamma)=0$. In this case, $\gamma$ need not have a representing measure, as we show in Example 2.5. If, however, $\gamma$ does have a representing measure $\mu$, then (1.7) implies $\operatorname{supp} \mu=\mathcal{V}(\gamma)$, and $M(n+1)[\mu]$ is a flat extension of $M(n)$, since 
$\operatorname{rank} M(n) \leq \operatorname{rank} M(n+1)[\mu] \leq \operatorname{card} \operatorname{supp} \mu \leq \operatorname{card} \mathcal{V}(\gamma)=\operatorname{rank} M(n)$. In addition, if $r:=\operatorname{rank} M(n) \leq 2 n+1$, then $\mu$ is the unique representing measure; indeed, the Vandermonde equation (1.3) determines the densities $\rho_{0}, \ldots, \rho_{r-1}$ uniquely in case the data $\left(\gamma_{0,0}, \ldots, \gamma_{0, r-1}\right)^{T}$ pertains entirely to the original moment problem, which occurs when $r-1 \leq 2 n$. For a simple illustration, consider $n=1$ and $M(1) \geq 0 . M(1)$ is recursively generated (vacuously) and $\operatorname{rank} M(1)=\operatorname{card} \mathcal{V}(\gamma)$ can occur only when $r=1$, i.e., $Z=\alpha 1$ for some $\alpha \in \mathbb{C}$ (cf. [CuF4, Theorem 6.1]). The associated representing measure, $\mu:=\gamma_{00} \delta_{\alpha}$, is of course unique in this case. For a richer illustration, consider Example 4.9, in which we construct a minimal representing measure in a case in which $M(3)$ is positive and recursively generated, and $\operatorname{rank} M(3)=\operatorname{card} \mathcal{V}(\gamma)=7$. Example 4.10 illustrates a case where $\rho(\gamma)=0$ and rank $M(n)>2 n+1$, but $\gamma$ again has a unique representing measure.

The case when $M(n)$ is positive, recursively generated, and $\rho(\gamma)>0$ also exhibits diverse possibilities. In the example cited above of an invertible $M(3)$ having no representing measure, we clearly have $\mathcal{V}(\gamma)=\mathbb{C}$. On the other hand, if $M(1) \geq 0$ and $\operatorname{rank} M(1)=2$, then $\mathcal{V}(\gamma)$ is a straight line which parameterizes infinitely many 2-atomic representing measures [CuF4, Theorem 6.1]; similarly, if $M(1)$ is positive and invertible, then $\mathcal{V}(\gamma)=\mathbb{C}$ and there exist infinitely many 3 -atomic representing measures [CuF4, Theorem 6.1].

Our next goal is to describe obstructions to positive extensions $M(n+1)$ related to the "functional calculus" $p \mapsto p(Z, \bar{Z})$ from $\mathcal{P}_{n}$ to $\mathcal{C}_{M(n)}$. We first recall some elements of the theory of positive extensions of moment matrices.

\section{Proposition 1.7.}

(i) $[$ CuF5, Lemma 1.9] Suppose $M(n)(\gamma)$ is positive and recursively generated. If $M(n+1)$ is a flat extension of $M(n)$, then $M(n+1)$ is positive and recursively generated.

(ii) (cf. Theorem 1.6) Suppose $M(n+1)$ is a positive extension of $M(n)$. If $p, q, p q \in \mathcal{P}_{n+1}$ and $p(Z, \bar{Z})=\mathbf{0}$, then $[p q(Z, \bar{Z})]_{(n)}=\mathbf{0}$.

Given $\gamma \equiv \gamma^{(2 n)}$, in addition to $M(n)$ we may also define blocks $B_{0, n+1}, \ldots$, $B_{n-1, n+1}$ via the obvious analogue of (1.2), that is,

$$
B_{i, n+1}:=\left(\gamma_{i+t-s, n+1+s-t}\right)_{0 \leq s \leq i} ; 0 \leq t \leq n+1 .
$$

Now, if we are given a block $B[n, n+1] \in M_{n+1, n+2}(\mathbb{C})$, let

$$
B:=\left(\begin{array}{c}
B_{0, n+1} \\
\vdots \\
B_{n-1, n+1} \\
B[n, n+1]
\end{array}\right)
$$

and denote the successive columns of $B$ by $Z^{n+1}, \bar{Z} Z^{n}, \ldots, \bar{Z}^{n+1}$, and the successive rows by $1, Z, \bar{Z}, \ldots, Z^{n}, \ldots, \bar{Z}^{n}$. For $0 \leq i+j \leq n, k+\ell=n+1$, we denote the row $\bar{Z}^{i} Z^{j}$, column $\bar{Z}^{k} Z^{\ell}$ entry of $B$ by $\left\langle\bar{z}^{k} z^{\ell}, \bar{z}^{i} z^{j}\right\rangle_{B}$; thus, for $0 \leq i+j \leq n-1$, we have $\left\langle\bar{z}^{k} z^{\ell}, \bar{z}^{i} z^{j}\right\rangle_{B}=\gamma_{k+j, \ell+i}$.

Following [CuF5], we say that $B[n, n+1]$ is symmetric if, whenever $i+j=n$, $k+\ell=n+1$, then $\left\langle\bar{z}^{k} z^{\ell}, \bar{z}^{i} z^{j}\right\rangle_{B}=\overline{\left\langle\bar{z}^{\ell} z^{k}, \bar{z}^{j} z^{i}\right\rangle_{B}}$; we also say that $B[n, n+1]$ satisfies normality if, whenever $i+j=n, k+\ell=n+1, j \geq 1$, and $\ell \geq 1$, then

$$
\left\langle\bar{z}^{k} z^{\ell}, \bar{z}^{i} z^{j}\right\rangle_{B}=\left\langle\bar{z}^{k+1} z^{\ell-1}, \bar{z}^{i+1} z^{j-1}\right\rangle_{B} .
$$


Recall from $[\mathbf{C u F 5},(1.11)-(1.13)]$ that to construct a flat moment matrix extension $M(n+1)$ of $M(n) \geq 0$ it is necessary and sufficient to construct a block $B[n, n+1]$ such that

$$
\begin{aligned}
& B[n, n+1] \text { is symmetric and satisfies normality; } \\
& \operatorname{Ran} B \subseteq \operatorname{Ran} M(n), \text { that is, } B=M(n) W \text { for some } W \\
& C:=W^{*} M(n) W \text { is Toeplitz, i.e., constant on diagonals: }
\end{aligned}
$$

indeed, in this case, a flat extension $M(n+1)$ is defined by

$$
[M(n) ; B]:=\left(\begin{array}{cc}
M(n) & B \\
B^{*} & C
\end{array}\right) .
$$

If, instead, a positive extension $M(n+1)$ is desired, the $C$ block in the above matrix must be a Toeplitz matrix satisfying $C \geq W^{*} M(n) W$.

In $[\mathbf{C u F 5}$ ] we were able to establish (1.9) in the following two cases that are independent of the case of flat data; both of these results play a role in our analysis of the quartic moment problem in Chapter 3.

Theorem 1.8. [CuF5, Theorem 2.1] Assume that $M(n)(\gamma)$ is positive, recursively generated, and satisfies $\bar{Z}=\alpha 1+\beta Z$ for some $\alpha, \beta \in \mathbb{C}$. Then $M(n)$ admits infinitely many flat extensions $M(n+1)$, each corresponding to a distinct minimal representing measure for $\gamma$.

Theorem 1.9. [CuF5, Theorem 3.1] Suppose $M(n)(\gamma)$ is positive and recursively generated. If $1 \leq k \leq\left[\frac{n}{2}\right]+1$ and $Z^{k}=p(Z, \bar{Z})$ for some $p \in \mathcal{P}_{k-1}$, then $M(n)$ admits a unique flat extension $M(n+1)$, which corresponds to the unique minimal representing measure for $\gamma$.

We show in Example 2.14 below that the restriction on $k$ in Theorem 1.9 is necessary.

Suppose now that $0 \leq i+j \leq n$ and $0 \leq k+\ell \leq n$, and suppose that there exist $p \in \mathcal{P}_{i+j-1}, q \in \mathcal{P}_{k+\ell-1}$ such that $\bar{Z}^{i} Z^{j}=p(Z, \bar{Z})$ and $\bar{Z}^{k} Z^{\ell}=q(Z, \bar{Z})$. Suppose also that there exist $r, s, t, u \geq 0$ such that $i+r=k+t, j+s=\ell+u$, and $i+r+j+s=n+1$. If $M(n)$ admits a recursively generated extension $M(n+1)$, then in $\mathcal{C}_{M(n+1)}$ we must have $\left(\bar{z}^{r} z^{s} p\right)(Z, \bar{Z})=\bar{Z}^{i+r} Z^{j+s}=\bar{Z}^{k+t} Z^{\ell+u}=$ $\left(\bar{z}^{t} z^{u} q\right)(Z, \bar{Z})$. In Example 2.2 we exhibit a positive, recursively generated $M(4)$ for which $\left(\bar{z}^{r} z^{s} p\right)(Z, \bar{Z}) \neq\left(\bar{z}^{t} z^{u} q\right)(Z, \bar{Z})$ in $\mathcal{C}_{M(4)}$; it follows from Proposition 1.7(ii) that this lack of recursive consistency is an obstruction to the existence of any positive extension $M(5)$. Hence, such an $M(4)$ does not admit any finitely atomic representing measure (by Theorem 1.5).

Now assume that $M(n)$ is positive, recursively generated and recursively consistent. Assume also that $i+j=n$ and that there exists $p \in \mathcal{P}_{n-1}$ such that

$$
\bar{Z}^{i} Z^{j}=p(Z, \bar{Z}) \text {. }
$$

Proposition 1.7(ii) implies that if $M(n)$ has a positive extension $M(n+1)$, then we must have

$$
\left[\bar{Z}^{i} Z^{j+1}\right]_{(n)}=[(z p)(Z, \bar{Z})]_{(n)}
$$

and

$$
\left[\bar{Z}^{i+1} Z^{j}\right]_{(n)}=[(\bar{z} p)(Z, \bar{Z})]_{(n)} .
$$


Thus,

$$
\left((z p)(Z, \bar{Z}), \widehat{\bar{z}^{k} z^{\ell}}\right)=\left((\bar{z} p)(Z, \bar{Z}), \widehat{\bar{z}^{k+1} z^{\ell-1}}\right)
$$

for $k \geq 0, \ell \geq 1$, and $k+\ell=n$ (by (1.8)). In Example 2.5 (with $n=4$ ) we construct a positive, recursively generated, recursively consistent $M(4)$ for which there exists a relation as in (1.10), and there exist $k \geq 0, \ell \geq 1$ with $k+\ell=n$, such that $\left((z p)(Z, \bar{Z}), \widehat{\bar{z}^{k} z^{\ell}}\right) \neq\left((\bar{z} p)(Z, \bar{Z}), \widehat{\bar{z}^{k+1} z^{\ell}-1}\right)$. This lack of normal consistency is an obstruction to any positive extension $M(5)$ : it is impossible to construct a block $B[n, n+1]$ satisfying $(1.8)$ if $M(n+1)$ is to be a positive extension.

Our main technical result, Theorem 2.7, establishes a natural class of moment matrices $M(n)$ for which positivity, recursive generation, recursive consistency and normal consistency imply

There exists a block $B[n, n+1]$ that is symmetric and satisfies normality, and for which $\operatorname{Ran} B \subseteq \operatorname{Ran} M(n)$.

If (1.11) holds, then (from (1.9)) to construct a flat extension $M(n+1)$, it suffices to verify that the corresponding self-adjoint block $C$ is Toeplitz. In principle, this entails $(n+1)(n+2) / 2$ verifications of diagonal relations of the form $C_{i, j}=C_{i+1, j+1}$, but in practice a much smaller number of tests suffices. The class of moment problems that we consider in Theorem 2.7 is defined as follows:

$$
\begin{aligned}
& \text { There is a basis for } \mathcal{C}_{M(n)} \text { of the form }\left\{\bar{Z}^{k} Z^{\ell}\right\}_{k, \ell \in I} \text { such that if } \\
& 0 \leq i+j \leq n \text { and }(i, j) \notin I \text {, then } \bar{Z}^{i} Z^{j}=\sum_{\substack{\ell+k<i+j \\
(\ell, k) \in I}} a_{\ell k} \bar{Z}^{\ell} Z^{k} .
\end{aligned}
$$

In the sequel we also present examples which do not satisfy (1.12) but for which we can nevertheless establish (1.11) and, further, prove the existence of flat extensions (e.g., Examples 4.9 and 4.10). Among the results outside the scope of Theorem 2.7 is also the following partial solution to the quartic moment problem that we prove in Chapter 3.

THEOREM 1.10. Suppose $M(2)(\gamma)$ is positive and recursively generated. Then $\gamma$ has a rank $M(2)$-atomic representing measure in each of the following cases:

(i) $\left\{1, Z, \bar{Z}, Z^{2}\right\}$ is linearly dependent in $\mathcal{C}_{M(2)}$;

(ii) $\left\{1, Z, \bar{Z}, Z^{2}\right\}$ is a basis for $\mathcal{C}_{M(2)}, \bar{Z} Z \in\langle 1, Z, \bar{Z}\rangle$, and the moments $\gamma_{i j}$ are all real, with the possible exception of $\gamma_{04}$;

(iii) $\left\{1, Z, \bar{Z}, Z^{2}\right\}$ is a basis for $\mathcal{C}_{M(2)}, \bar{Z} Z \in\langle 1, Z, \bar{Z}\rangle$, and the reduced $C$-block test $C_{11}=C_{22}$ passes;

(iv) $\left\{1, Z, \bar{Z}, Z^{2}, \bar{Z}^{2}\right\}$ is a basis for $\mathcal{C}_{M(2)}, \bar{Z} Z \in\langle 1, Z, \bar{Z}\rangle$, and the reduced $C$ block test $C_{11}=C_{22}$ passes for some choice of $\gamma_{05}$.

In an Appendix we include a table summarizing many of the examples and theorems of the present work and of $[\mathbf{C u F} 4]$ and $[\mathbf{C u F 5}]$. Analysis of this table leads us to observe that for fixed $n$, as the number of recursive relations in $\mathcal{C}_{M(n)}$ increases, the easier it is to determine whether or not $M(n)$ admits a flat extension $M(n+1)$. Indeed, each such relation is of the form $\bar{Z}^{i} Z^{j}=p(Z, \bar{Z})$, with $\operatorname{deg} p<$ $i+j \leq n$; as the relation propagates recursively, it uniquely determines various columns of any proposed moment matrix extension block $B$ which is to satisfy (1.11). Assuming such a block $B$ exists, the recursively determined columns of $B$ also serve to simplify the test for normality in the $C$ block of $[M(n) ; B]$ (cf. Proposition 2.6). 
Acknowledgments. The authors wish to thank Professors Jim Agler, Edwin Franks, Palle Jørgensen, John E. McCarthy, Mihai Putinar, Bruce Reznick, Mark Stankus, Jan Stochel and Franek Szafraniec, and the referee, for helpful comments and discussions concerning the subject of this paper. Special thanks are also due to Brian Treadway, who contributed extensively with technical typing and proofreading; in addition, he detected an error in the original version of Example 4.7. Many of the calculations in Chapters 2, 3 and 4 have been obtained through computer experiments using the software tool Mathematica [Wol]. 


\section{CHAPTER 2}

\section{Flat Extensions for Moment Matrices}

In this chapter we develop conditions for the existence of flat extensions $M(n+1)$ of a positive, recursively generated moment matrix $M(n) \equiv M(n)(\gamma)$. The main result, Theorem 2.7, leads to a computational procedure for constructing flat extensions using only elementary linear algebra.

Throughout this chapter $M(n)$ denotes a positive, recursively generated moment matrix; by a positive structured extension of $M(n)$ we mean a positive matrix $M \in M_{\frac{(n+2)(n+3)}{2}}(\mathbb{C})$ which admits a block decomposition

$$
M=\left(\begin{array}{cc}
M(n) & B \\
B^{*} & C
\end{array}\right)
$$

where

$$
B=\left(\begin{array}{c}
B_{0, n+1}(\gamma) \\
\vdots \\
B_{n-1, n+1}(\gamma) \\
B[n, n+1]
\end{array}\right), \quad B[n, n+1] \in M_{n+1, n+2}(\mathbb{C})
$$

and

$$
C \in M_{n+2, n+2}(\mathbb{C}) .
$$

We refer to block $B$ in (2.2) as an extension block for $M(n)$. If, additionally, $B[n, n+1]$ has the form of a moment matrix block, then we refer to $B$ as a moment matrix extension block.

More generally, for a positive matrix $A$, an extension of $A$ is a block matrix of the form

$$
\tilde{A} \equiv\left(\begin{array}{cc}
A & B \\
B^{*} & C
\end{array}\right)
$$

A result of Smul'jan [Smu] implies that $\tilde{A} \geq 0$ if and only if (i) $A \geq 0$, (ii) there exists a matrix $W$ such that $B=A W$ (equivalently, $\operatorname{Ran} B \subseteq \operatorname{Ran} A[$ Dou]), and (iii) $C \geq W^{*} A W$. (Note that in this case $W^{*} A W$ is independent of $W$ satisfying $A W=B$.) The Extension Principle [Fia1] shows that if $\tilde{A} \geq 0$, then each dependence relation in the columns of $A$ extends to a corresponding dependence relation in the columns of $\tilde{A}$.

By a flat extension of $A$ we mean an extension $\tilde{A}$ satisfying $\operatorname{rank} \tilde{A}=\operatorname{rank} A$.

Proposition 2.1. Let $A \geq 0 . \tilde{A}$ is a flat extension of $A$ if and only if $B=A W$ and $C=W^{*} A W$ for some matrix $W$, in which case $\tilde{A} \geq 0$. 
A positive structured extension $M$ (as in $(2.1)-(2.3)$ ) is flat if $\operatorname{rank} M=$ rank $M(n)$; from Proposition 2.1, this entails

$$
\left(\begin{array}{l}
B \\
C
\end{array}\right)=\left(\begin{array}{c}
M(n) \\
B^{*}
\end{array}\right) W
$$

or, equivalently, $B=M(n) W$ and $C=W^{*} M(n) W$. Thus, a flat extension $M$ is completely determined by the choice of a block $B[n, n+1]$ satisfying $\operatorname{Ran} B \subseteq$ $\operatorname{Ran} M(n)$; we denote such an extension by

$$
M \equiv[M(n) ; B]
$$

Constructing $[M(n) ; B]$ so that it is a moment matrix is by no means straightforward. To construct a flat moment matrix extension $M(n+1)$ of $M(n)$ it is necessary and sufficient to identify new moments of order $2 n+1$,

$$
\tilde{\gamma}: \gamma_{0,2 n+1}, \ldots, \gamma_{2 n+1,0}, \gamma_{i j}=\overline{\gamma_{j i}}
$$

such that the block $B[n, n+1] \equiv B_{n, n+1}(\tilde{\gamma})$ satisfies

$$
\operatorname{Ran} B \subseteq \operatorname{Ran} M(n)
$$

and

$$
\text { the } C \text { block of }[M(n) ; B] \text { is Toeplitz. }
$$

Let $M(n+1)$ denote a positive moment matrix extension of a (positive) recursively generated moment matrix $M(n)$. Suppose that there exist $i, j \geq 0, i+j=n$, and polynomials $p, q \in \mathcal{P}_{n-1}$ such that

$$
\bar{Z}^{i} Z^{j}=p(Z, \bar{Z}) \quad \text { and } \quad \bar{Z}^{i+1} Z^{j-1}=q(Z, \bar{Z}) \quad \text { in } \quad \mathcal{C}_{M(n)}
$$

In $\mathcal{C}_{M(n+1)}$ we must have

$$
[(\bar{z} p)(Z, \bar{Z})]_{(n)}=\left[\bar{Z}^{i+1} Z^{j}\right]_{(n)}=[(z q)(Z, \bar{Z})]_{(n)}
$$

(by Proposition 1.7(ii)); in particular,

$$
[(\bar{z} p)(Z, \bar{Z})]_{(n)}=[(z q)(Z, \bar{Z})]_{(n)}
$$

We seek to exhibit a positive, recursively generated moment matrix $M(n)$ for which (2.7) fails; this will lead to the notion of recursive consistency, which is necessary for the existence of positive extensions $M(n+1)$. We first introduce a family of moment matrices that will be used in several examples in the sequel. 
Define $M(4)$ by

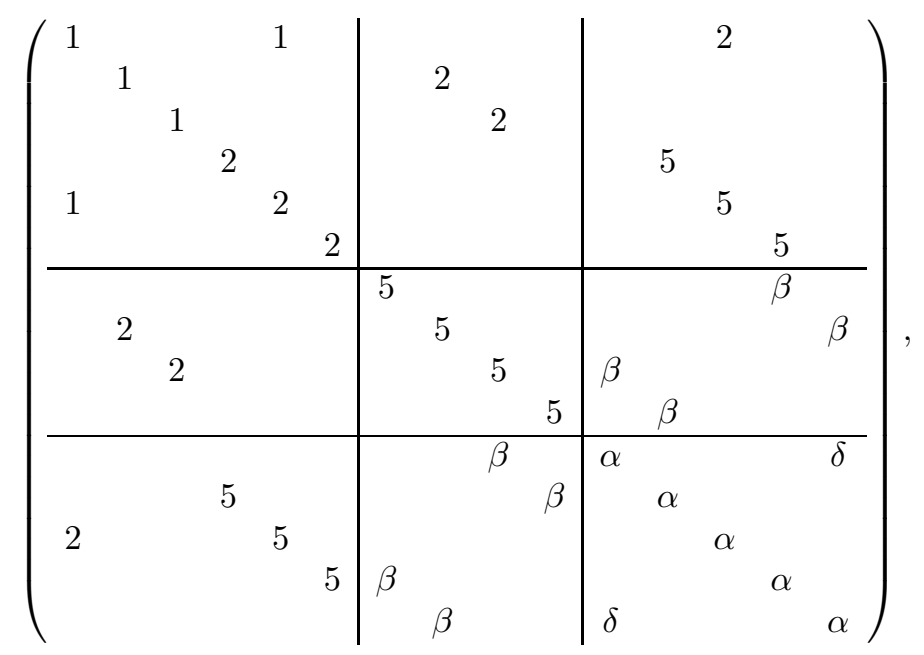

where $\alpha, \beta$ and $\delta$ are real parameters whose values will be specified later, and where all blanks represent zeros. Write $M(4)$ in block form as

$$
\left(\begin{array}{cc}
M(3) & B \\
B^{*} & C
\end{array}\right) .
$$

To show that $M(4) \geq 0$, we prove that $M(3) \geq 0, B=M(3) W$ for some $W$, and $C \geq W^{*} B$. The Nested Determinants Test shows at once that $M(3)$ is positive and invertible (in symbols, $M(3)>0$ ). Let $W=M(3)^{-1} B$; a calculation shows that $\triangle:=C-W^{*} M(3) W$ is equal to

$$
\left(\begin{array}{ccccc}
\alpha-\beta^{2} & 0 & 0 & 0 & \delta \\
0 & \alpha-\left(\frac{25}{2}+\frac{\beta^{2}}{5}\right) & 0 & 0 & 0 \\
0 & 0 & \alpha-13 & 0 & 0 \\
0 & 0 & 0 & \alpha-\left(\frac{25}{2}+\frac{\beta^{2}}{5}\right) & 0 \\
\delta & 0 & 0 & 0 & \alpha-\beta^{2}
\end{array}\right) .
$$

We set $\alpha=\frac{25}{2}+\frac{\beta^{2}}{5}$ and we further require $\alpha>\beta^{2}$ and $\alpha \geq 13$; thus if $|\delta| \leq \alpha-\beta^{2}$, then $\triangle \geq 0$, whence $M(4) \geq 0$. Note that since $M(3)>0, M(4)$ is automatically recursively generated. The condition $\alpha>\beta^{2}$ insures that column $Z^{4}$ is independent of all columns to its left.

We also have

$$
Z^{3} \bar{Z}=\frac{5}{2} Z^{2}+\frac{\beta}{5} \bar{Z}^{3}
$$

and

$$
\bar{Z}^{3} Z=\frac{5}{2} \bar{Z}^{2}+\frac{\beta}{5} Z^{3}
$$

in $\mathcal{C}_{M(4)}$, so $11 \leq \operatorname{rank} M(4) \leq 13$ 
Example 2.2. Let $\alpha=13, \beta=\sqrt{\frac{5}{2}}, \delta=\alpha-\beta^{2}=\frac{21}{2}$. Then $M(4) \geq 0$ is recursively generated and in $\mathcal{C}_{M(4)}$ we have

$$
\bar{Z} Z^{3}=\frac{5}{2} Z^{2}+\frac{1}{\sqrt{10}} \bar{Z}^{3}
$$

and

$$
\bar{Z}^{2} Z^{2}=-1+3 \bar{Z} Z
$$

If (2.7) were to hold, in $\mathcal{C}_{M(4)}$ we would then have

$$
\frac{5}{2} \bar{Z} Z^{2}+\frac{1}{\sqrt{10}} \bar{Z}^{4}=-Z+3 \bar{Z} Z^{2},
$$

or equivalently,

$$
\bar{Z}^{4}=-\sqrt{10} Z+\sqrt{\frac{5}{2}} \bar{Z} Z^{2} .
$$

However, observe that $\left\langle\bar{z}^{4}, \bar{z}^{4}\right\rangle_{M(4)}=\alpha \neq \beta^{2}=\left\langle-\sqrt{10} z+\sqrt{\frac{5}{2}} \bar{z} z^{2}, \bar{z}^{4}\right\rangle_{M(4)}$, so (2.9) cannot hold; thus $M(4)$ admits no positive extension $M(5)$. (In Example 4.6 we show that $\gamma$ actually admits no representing measure.)

In view of Example 2.2 we introduce the following terminology. A recursive relation in $\mathcal{C}_{M(n)}$ is a triple $(i, j, p)$ such that $i, j \geq 0, p \in \mathcal{P}_{n-1}$, deg $p<i+j \leq n$, and

$$
\bar{Z}^{i} Z^{j}=p(Z, \bar{Z})
$$

we also say that $\bar{Z}^{i} Z^{j}$ is recursively determined. Since $M(n)$ is recursively generated, it then follows that for all $r, s \geq 0$ with $i+r+j+s \leq n$,

$$
\bar{Z}^{i+r} Z^{j+s}=\left(\bar{z}^{r} z^{s} p\right)(Z, \bar{Z}) .
$$

Let $k, \ell \geq 0, k+\ell=n+1$. An element $\mathbf{v} \in \mathcal{C}_{M(n)}$ is recursively determined with type $(k, \ell)$ if there exists a recursive relation $(i, j, p)$ such that $i \leq k, j \leq \ell$, and $\mathbf{v}=\left(\bar{z}^{k-i} z^{\ell-j} p\right)(Z, \bar{Z})$; the set of all such vectors $\mathbf{v}$ will be denoted by $\mathcal{R}(k, \ell)$. We say that $\mathbf{v} \in \mathcal{C}_{M(n)}$ is admissible for $M(n)$ if $\mathbf{v}$ is recursively determined with type $(k, \ell)$ and $\operatorname{card} \mathcal{R}(k, \ell)=1$. Thus, if $\mathbf{v}$ is admissible and $(i, j, p)$ and $\left(i^{\prime}, j^{\prime}, p^{\prime}\right)$ are two recursive relations with $i, i^{\prime} \leq k, j, j^{\prime} \leq \ell$, then $\left(\bar{z}^{k-i} z^{\ell-j} p\right)(Z, \bar{Z})=\left(\bar{z}^{k-i^{\prime}} z^{\ell-j^{\prime}} p^{\prime}\right)(Z, \bar{Z})=\mathbf{v}$; we denote this common value by $\bar{Z}^{k} Z^{\ell}$. In this case, if $M(n+1)$ is a positive moment matrix extension of $M(n)$, with columns labeled $\overline{\tilde{Z}}^{i} \tilde{Z}^{j}$, then

$$
\left[\overline{\tilde{Z}}^{k} \tilde{Z}^{\ell}\right]_{(n)}=\bar{Z}^{k} Z^{\ell} \in \mathcal{C}_{M(n)}
$$

(recall that $[\mathbf{w}]_{(d)}$ denotes the truncation of the vector $\mathbf{w} \in \mathbb{C}^{m(n)}$ through entries of degree at most $d$ ). For an admissible column $\bar{Z}^{k} Z^{\ell}$ and for $0 \leq u+v \leq n$, we denote the $\bar{Z}^{u} Z^{v}$ component of $\bar{Z}^{k} Z^{\ell}$ by $\left\langle\bar{z}^{k} z^{\ell}, \bar{z}^{u} z^{v}\right\rangle_{B}$. Note that for $0 \leq u+v \leq n-1$,

$$
\left\langle\bar{z}^{k} z^{\ell}, \bar{z}^{u} z^{v}\right\rangle_{B}=\gamma_{k+v, \ell+u}
$$

(this follows from the Proof of Theorem 1.6; cf. [CuF4, (5.2)]).

Example 2.2 shows that the following is a nontrivial necessary condition for the existence of a positive extension $M(n+1)$ of a positive recursively generated moment matrix $M(n)$ : 
$(\mathbf{R C})$

If $k, \ell \geq 0, k+\ell=n+1$, and if $\mathcal{R}(k, \ell) \neq \varnothing$, then $\bar{Z}^{k} Z^{\ell}$ is admissible for $M(n)$.

Observe that $(\mathbf{R C})$ is necessary for the existence of a representing measure with moments up to order $2(n+1)$ (cf. Theorem 1.5). If property (RC) holds for a positive recursively generated $M(n)$, we say that $M(n)$ is recursively consistent. In this case, for a recursive relation $(i, j, p)$, each column in the set

$$
\mathcal{T}(i, j, p):=\left\{\bar{Z}^{i} Z^{n+1-i}, \ldots, \bar{Z}^{n+1-j} Z^{j}\right\}
$$

is admissible.

Proposition 2.3. If $M(n)$ is recursively consistent, then for each relation $(i, j, p)$, the successive columns $\mathcal{T}(i, j, p)$ given by (2.11) form a normal band, i.e., if $t+u \leq n, u \geq 1,0 \leq r \leq n-i$, then

$$
\left\langle\bar{z}^{i+r} z^{n+1-i-r}, \bar{z}^{t} z^{u}\right\rangle_{B}=\left\langle\bar{z}^{i+r+1} z^{n-i-r}, \bar{z}^{t+1} z^{u-1}\right\rangle_{B} .
$$

Proof. Since $\bar{Z}^{i} Z^{j}=p(Z, \bar{Z})$, then

$$
\bar{Z}^{i+r} Z^{n+1-i-r}=\left(\bar{z}^{r} z^{n+1-i-r-j} p\right)(Z, \bar{Z})
$$

and

$$
\bar{Z}^{i+r+1} Z^{n-i-r}=\left(\bar{z}^{r+1} z^{n-i-r-j} p\right)(Z, \bar{Z}) .
$$

Now $r+(n+1-i-r-j)+\operatorname{deg} p<n+1$, so

$$
\begin{aligned}
\left\langle\bar{z}^{i+r} z^{n+1-i-r}, \bar{z}^{t} z^{u}\right\rangle_{B} & =\left\langle\bar{z}^{r} z^{n+1-i-r-j} p, \bar{z}^{t} z^{u}\right\rangle_{M(n)} \\
& =\left\langle\bar{z}^{r+1} z^{n-i-r-j} p, \bar{z}^{t+1} z^{u-1}\right\rangle_{M(n)}
\end{aligned}
$$

(by normality in $M(n)[\mathbf{C u F} 4$, Theorem 2.1(4)])

$$
=\left\langle\bar{z}^{i+r+1} z^{n-i-r}, \bar{z}^{t+1} z^{u-1}\right\rangle_{B} .
$$

Admissibility is also invariant under "conjugation," as the next result shows.

Proposition 2.4. Let $k+\ell=n+1$ and suppose $\bar{Z}^{k} Z^{\ell}$ is admissible. Then $\bar{Z}^{\ell} Z^{k}$ is admissible, and is symmetric with respect to $\bar{Z}^{k} Z^{\ell}$, i.e., for $0 \leq u+v \leq n$,

$$
\left\langle\bar{z}^{k} z^{\ell}, \bar{z}^{u} z^{v}\right\rangle_{B}=\overline{\left\langle\bar{z}^{\ell} z^{k}, \bar{z}^{v} z^{u}\right\rangle_{B}} .
$$

Proof. Suppose $\bar{Z}^{k} Z^{\ell}$ is recursively generated from $(i, j, p)$, i.e., $\bar{Z}^{k} Z^{\ell}=$ $\left(\bar{z}^{r} z^{s} p\right)(Z, \bar{Z})$ for some $r, s \geq 0$. It follows readily from [CuF4, Lemma 3.10] that $\bar{Z}^{\ell} Z^{k}$ is admissible and that $\bar{Z}^{\ell} Z^{k}=\left(\bar{z}^{s} z^{r} \bar{p}\right)(Z, \bar{Z})$. Now

$$
\begin{aligned}
\left\langle\bar{z}^{k} z^{\ell}, \bar{z}^{u} z^{v}\right\rangle_{B}= & \left\langle\bar{z}^{r} z^{s} p, \bar{z}^{u} z^{v}\right\rangle_{M(n)} \\
= & \left\langle\bar{z}^{v} z^{u}, \bar{z}^{s} z^{r} \bar{p}\right\rangle_{M(n)} \\
& \quad \frac{(\text { by symmetry in }}{\left\langle\bar{z}^{s} z^{r} \bar{p}, \bar{z}^{v} z^{u}\right\rangle_{M(n)}} \\
= & \overline{\left\langle\bar{z}^{\ell} z^{k}, \bar{z}^{v} z^{u}\right\rangle_{B} .}
\end{aligned}
$$

(by symmetry in $M(n)[\mathbf{C u F 4}$, Theorem 2.1(2)])

Suppose $M(n)$ is recursively consistent. Let $k+\ell=n+1$ and suppose $\bar{Z}^{k} Z^{\ell}$ is admissible. If $\bar{Z}^{k} Z^{\ell}$ is part of normal bands $\mathcal{T}$ and $\mathcal{T}^{\prime}$, then it follows that $\mathcal{T} \cup \mathcal{T}^{\prime}$ is a normal band. On the other hand, it is possible for $\bar{Z}^{k} Z^{\ell}$ and $\bar{Z}^{k+1} Z^{\ell-1}$ to be admissible without forming a normal band, as the next example illustrates. 
EXAmPle 2.5. We define $M(4)$ via (2.8) using $\alpha=\frac{143}{10}, \beta=3, \delta=5$. We have $\alpha>\beta^{2}, \alpha>13$, and $\delta<\alpha-\beta^{2}$, whence $M(4) \geq 0$ and $\operatorname{rank} M(4)=13$. The only recursive relations in $M(4)$ are

$$
\bar{Z} Z^{3}=\frac{5}{2} Z^{2}+\frac{3}{5} \bar{Z}^{3} \quad \text { and } \quad \bar{Z}^{3} Z=\frac{5}{2} \bar{Z}^{2}+\frac{3}{5} Z^{3} .
$$

These relations propagate consistently to the admissible columns

$$
\bar{Z}^{2} Z^{3}:=\frac{5}{2} \bar{Z} Z^{2}+\frac{3}{5} \bar{Z}^{4}
$$

and

$$
\bar{Z}^{3} Z^{2}:=\frac{5}{2} \bar{Z}^{2} Z+\frac{3}{5} Z^{4}
$$

Direct calculation shows that

$$
\left\langle\bar{z}^{2} z^{3}, z^{4}\right\rangle_{B}=\frac{3}{5} \delta \neq 0=\left\langle\bar{z}^{3} z^{2}, \bar{z} z^{3}\right\rangle_{B}
$$

so $\bar{Z}^{2} Z^{3}$ and $\bar{Z}^{3} Z^{2}$ are not normal with respect to each other. Thus $M(4)$ admits no positive extension $M(5)$, and, in particular, no flat moment matrix extension. (In Example 4.7 we show that $\gamma$ actually admits no representing measure.)

In view of this example, the following property is a nontrivial necessary condition for positive extensions of positive recursively generated moment matrices (or, more generally, for representing measures with moments up to order $2(n+1)$ [CuF4, Corollary 3.4], [Fia1]; cf. Theorem 1.5).

$(\mathrm{NC})$

If $k, \ell \geq 0, k+\ell=n+1$, and $\bar{Z}^{k} Z^{\ell}$ and $\bar{Z}^{k+r} Z^{\ell-r}$ are admissible, then they are normal with respect to each other, i.e.,

$$
\left\langle\bar{z}^{k} z^{\ell}, \bar{z}^{s} z^{t}\right\rangle_{B}=\left\langle\bar{z}^{k+r} z^{\ell-r}, \bar{z}^{s+r} z^{t-r}\right\rangle_{B}
$$

whenever $s+t \leq n$ with $s, t, s+r, s-r \geq 0$.

(The identity in (NC) always holds for $s+t \leq n-1$ [CuF4, (5.1), (5.2)].) If property (NC) holds for all pairs of admissible columns $\bar{Z}^{k} Z^{\ell}$ and $\bar{Z}^{k+r} Z^{\ell-r}$, then we say that $M(n)$ is normally consistent. The next result shows that normal consistency propagates to the $C$ block of $[M(n) ; B]$.

Proposition 2.6. Let $k+\ell=n+1, \ell \geq 1$, suppose $\bar{Z}^{i} Z^{j}=p(Z, \bar{Z})$ in $\mathcal{C}_{M(n)}$ with $\operatorname{deg} p<i+j \leq n$, and suppose $(i, j, p) \in \mathcal{R}(k, \ell) \cap \mathcal{R}(k+1, \ell-1)$. Assume further that $\bar{Z}^{k} Z^{\ell}:=\left(\bar{z}^{k-i} z^{\ell-j} p\right)(Z, \bar{Z})$ and $\bar{Z}^{k+1} Z^{\ell-1}:=\left(\bar{z}^{k-i+1} z^{\ell-j-1} p\right)(Z, \bar{Z})$ belong to an extension block $B$ for which $B[n, n+1]$ satisfies normality and such that $\operatorname{Ran} B \subseteq \operatorname{Ran} M(n)$. Then in the $C$ block of $M \equiv[M(n) ; B], \tilde{\bar{Z}}^{k} \tilde{Z}^{\ell}$ and $\tilde{\bar{Z}}^{k+1} \tilde{Z}^{\ell-1}$ are normal with respect to each other.

Proof. Let $r \geq 0, s \geq 1$ be such that $\bar{Z}^{k} Z^{\ell}=\left(\bar{z}^{r} z^{s} p\right)(Z, \bar{Z})$ and $\bar{Z}^{k+1} Z^{\ell-1}=$ $\left(\bar{z}^{r+1} z^{s-1} p\right)(Z, \bar{Z})$, and let $d:=\operatorname{deg} p$; we have $p(z, \bar{z})=\sum_{0 \leq u+v \leq d} a_{u v} \bar{z}^{u} z^{v}$. Let $x+y=$
$n+1, y \geq 1$; we must show that

$$
\left\langle\bar{z}^{k} z^{\ell}, \bar{z}^{x} z^{y}\right\rangle_{M}=\left\langle\bar{z}^{k+1} z^{\ell-1}, \bar{z}^{x+1} z^{y-1}\right\rangle_{M} .
$$

Now

$$
\left\langle\bar{z}^{k} z^{\ell}, \bar{z}^{x} z^{y}\right\rangle_{M}=\left\langle\sum_{u, v} a_{u v} \bar{z}^{u+r} z^{v+s}, \bar{z}^{x} z^{y}\right\rangle_{M}=\sum_{u, v} a_{u v}\left\langle\bar{z}^{u+r} z^{v+s}, \bar{z}^{x} z^{y}\right\rangle_{M}
$$


where

$$
u+r+v+s \leq d+r+s<i+r+j+s=n+1 .
$$

Since the blocks $B_{j, n+1}$ satisfy normality $(j=0, \ldots, n)$, the blocks $B_{n+1, j} \equiv B_{j, n+1}^{*}$ also satisfy normality; thus

$$
\left\langle\bar{z}^{u+r} z^{v+s}, \bar{z}^{x} z^{y}\right\rangle_{M}=\left\langle\bar{z}^{u+r+1} z^{v+s-1}, \bar{z}^{x+1} z^{y-1}\right\rangle_{M}
$$

whence

$$
\begin{aligned}
\left\langle\bar{z}^{k} z^{\ell}, \bar{z}^{x} z^{y}\right\rangle & =\sum_{u, v} a_{u v}\left\langle\bar{z}^{u+r+1} z^{v+s-1}, \bar{z}^{x+1} z^{y-1}\right\rangle_{M} \\
& =\left\langle\bar{z}^{r+1} z^{s-1} p, \bar{z}^{x+1} z^{y-1}\right\rangle_{M}=\left\langle\bar{z}^{k+1} z^{\ell-1}, \bar{z}^{x+1} z^{y-1}\right\rangle_{M} .
\end{aligned}
$$

Recall that if $B$ is a moment matrix extension block satisfying $\operatorname{Ran} B \subseteq \operatorname{Ran} M(n)$, then $[M(n) ; B]$ is a moment matrix if and only if the $C$ block satisfies normality, i.e., is constant on diagonals. In principle, establishing this property entails $(n+1)^{2}$ tests of the form $C_{i, j}=C_{i+1, j+1}$, but several factors serve to reduce this number:

(i) $C$ is self-adjoint, so it suffices to consider the main diagonal and the lower diagonals;

(ii) $C$ is a symmetric block $[\mathbf{C u F 4}$, Proposition 2.3], so within each diagonal elements that are symmetric with respect to the diagonal midpoint are equal;

(iii) as described in Proposition 2.6, normal bands in the $B$ block propagate to normal bands in the $C$ block.

Conditions (i)-(iii) thus lead to a reduced $C$-block test for normality. To illustrate this test, consider $M(3) \geq 0$ satisfying (RG), (RC), (NC), and suppose $\bar{Z} Z^{2}=p(Z, \bar{Z})$, where $\operatorname{deg} p \leq 2$. Suppose in addition that there exists a moment matrix extension block $B$ satisfying $\operatorname{Ran} B \subseteq \operatorname{Ran} M(3)$. To establish normality in the $C$ block of $[M(3) ; B]$ it suffices to focus on the diagonals $C_{11}, \ldots, C_{55}$, $C_{21}, \ldots, C_{54}, C_{31}, \ldots, C_{53}$, and $C_{41}, \ldots, C_{52}$ (Condition (i)). From Condition (ii), we have $C_{11}=C_{55}, C_{22}=C_{44}, C_{21}=C_{54}, C_{32}=C_{43}, C_{31}=C_{53}$, and $C_{41}=C_{52}$. The relation $\bar{Z} Z^{2}=p(Z, \bar{Z})$ leads to a normal band $\bar{Z} Z^{3}, \bar{Z}^{2} Z^{2}, \bar{Z}^{3} Z$ (Proposition 2.3), which propagates into the $C$ block (Proposition 2.6), yielding $C_{22}=C_{33}=$ $C_{44}, C_{32}=C_{43}=C_{54}$, and $C_{42}=C_{53}$ (Condition (iii)). Thus the reduced $C$-block test for this moment problem is $C_{11}=C_{22}$.

The main result of this chapter, which follows, establishes moment matrix extension blocks $B$, with $\operatorname{Ran} B \subseteq \operatorname{Ran} M(n)$, for a large class of positive moment matrices $M(n)$ which satisfy $(\mathbf{R G}),(\mathbf{R C}),(\mathbf{N C})$. For this class, $M(n)$ has a flat extension if and only if $[M(n) ; B]$ satisfies the reduced $C$-block test.

TheOREM 2.7. Let $M(n)$ be positive, singular, recursively generated, recursively consistent, and normally consistent. Suppose either that $\bar{Z}^{k} Z^{\ell}$ is admissible for every $k+\ell=n+1$, or that there is a basis $\left\{\bar{Z}^{p} Z^{q}\right\}_{(p, q) \in I}$ for $\mathcal{C}_{M(n)}$ such that if $(r, s) \notin I$, then $\bar{Z}^{r} Z^{s}$ is recursively determined by basis elements of lower degree. Then there exists a moment matrix extension block $B$ satisfying $\operatorname{Ran} B \subseteq \operatorname{Ran} M(n)$; if the $C$ block of $[M(n) ; B]$ satisfies normality, then $M(n)$ has a flat extension $M(n+1):=\left(\begin{array}{cc}M(n) & B \\ B^{*} & C\end{array}\right)$. 
REMARK 2.8. We will actually give a complete parameterization of the moment matrix extension blocks $\left\{B_{\alpha}\right\}_{\alpha \in A}$ satisfying $\operatorname{Ran} B_{\alpha} \subseteq \operatorname{Ran} M(n)$; thus $M(n)$ admits a flat extension $M(n+1)$ if and only if for some $\alpha \in A$, the $C$ block of $\left[M(n), B_{\alpha}\right]$ is Toeplitz. Normality in the $C$ block can be verified using the reduced $C$-block test, as we illustrate in several examples. Theorem 2.7 also leads to a new proof that a flat, positive moment matrix $M(n)$ has a unique flat extension $M(n+1)$ (Proposition 2.12 below).

Proof of Theorem 2.7. Let $\mathcal{T}:=\{(i, j): i+j=n+1\}, \mathcal{R}:=\{(i, j) \in$ $\mathcal{T}: \bar{Z}^{i} Z^{j}$ is admissible $\}$, and $\mathcal{S}:=\mathcal{T} \backslash \mathcal{R}$. If $\mathcal{R}=\mathcal{T}$, then the existence of a unique moment matrix extension block $B$ satisfying $\operatorname{Ran} B \subseteq \operatorname{Ran} M(n)$ follows from recursive consistency, normal consistency, and symmetry (Proposition 2.4). We may therefore assume $\mathcal{R} \neq \mathcal{T}$; since $M(n)$ is singular, we must have $\mathcal{R} \neq \varnothing$. (Note that for $(i, j) \in \mathcal{R}$ and $0 \leq r+s \leq n-1,\left\langle\bar{z}^{i} z^{j}, \bar{z}^{r} z^{s}\right\rangle_{B}=\gamma_{i+s, j+r}$.) Our aim is to define a moment matrix extension block $B$, with $\operatorname{Ran} B \subseteq \operatorname{Ran} M(n)$, by using $\left\{\bar{Z}^{i} Z^{j}\right\}_{(i, j) \in \mathcal{R}}$ and by propagating these columns using normal consistency and symmetry.

We use an inductive procedure to define the non-admissible columns in such a way that the resulting moment matrix extension block $B$ satisfies Ran $B \subseteq$ $\operatorname{Ran} M(n)$. Let $\mathcal{S}^{\prime}=\left\{(i, j) \in \mathcal{S}: \bar{Z}^{i} Z^{j}\right.$ has been defined $\}\left(\mathcal{S}^{\prime}\right.$ is initially empty). Our inductive assumption is that the columns of $\mathcal{R} \cup \mathcal{S}^{\prime}$ are consistent with moment matrix structure, and we show how to extend this structure to a column $\bar{Z}^{i} Z^{j} \in$ $\operatorname{Ran} M(n)$ with $(i, j) \in \mathcal{S} \backslash \mathcal{S}^{\prime}$. We define $\bar{Z}^{i} Z^{j}$ by specifying all its components $\left\langle\bar{z}^{i} z^{j}, \bar{z}^{r} z^{s}\right\rangle_{B}(0 \leq r+s \leq n)$, and we consider three cases for $(r, s)$.

(i) For $(r, s) \in I, 0 \leq r+s \leq n-1$, let $\left\langle\bar{z}^{i} z^{j}, \bar{z}^{r} z^{s}\right\rangle_{B}:=\gamma_{i+s, j+r}$; it follows from $[\mathbf{C u F} 4,(5.1),(5.2)]$ that this is consistent with any value that could be assigned this entry by normal propagation from previously defined columns (admissible or non-admissible).

(ii) For $(r, s) \in I$ with $r+s=n$, if $\left\langle\bar{z}^{i} z^{j}, \bar{z}^{r} z^{s}\right\rangle_{B}$ is not already normally determined from some previously defined column, then this entry is free and we assign a value $\gamma_{i+s, j+r}$ arbitrarily.

(iii) For every $(r, s) \notin I$, we will show that $\left\langle\bar{z}^{i} z^{j}, \bar{z}^{r} z^{s}\right\rangle_{B}$ is normally determined from some admissible column; of course, if $r+s \leq n-1$, this implies $\left\langle\bar{z}^{i} z^{j}, \bar{z}^{r} z^{s}\right\rangle_{B}:=\gamma_{i+s, j+r}$.

Let $M_{I}$ denote the compression of $M(n)$ to the rows and columns indexed by $I$; $M_{I}>0$. Let $V=\left[\bar{Z}^{i} Z^{j}\right]_{I}$ (as defined above in (i) and (ii)); since $M_{I}$ is invertible, there exist unique scalars $a_{k \ell}((k, \ell) \in I)$ such that

$$
V=\sum_{(k, \ell) \in I} a_{k \ell}\left[\bar{Z}^{k} Z^{\ell}\right]_{I}
$$

i.e., for $(p, q) \in I$,

$$
\left\langle\bar{z}^{i} z^{j}, \bar{z}^{p} z^{q}\right\rangle_{B}=\sum_{(k, \ell) \in I} a_{k \ell} \gamma_{k+q, p+\ell}
$$

Thus, to complete $\bar{Z}^{i} Z^{j}$ (consistent with moment matrix block structure for block $B$ ) so that $\bar{Z}^{i} Z^{j} \in \operatorname{Ran} M(n)$, it is sufficient to establish the following. 
Claim. For $0 \leq r+s \leq n,(r, s) \notin I,\left\langle\bar{z}^{i} z^{j}, \bar{z}^{r} z^{s}\right\rangle_{B}$ is normally determined (from some admissible column), and equals

$$
\tau:=\sum_{(k, \ell) \in I} a_{k \ell}\left\langle\bar{z}^{k} z^{\ell}, \bar{z}^{r} z^{s}\right\rangle_{M(n)}=\sum_{(k, \ell) \in I} a_{k \ell} \gamma_{k+s, \ell+r} .
$$

Suppose $0 \leq r+s \leq n,(r, s) \notin I$. In $\mathcal{C}_{M(n)}$, we have the relation

$$
\bar{Z}^{r} Z^{s}=\sum_{\substack{0 \leq p+q<r+s \\(p, q) \in I}} \alpha_{p q} \bar{Z}^{p} Z^{q},
$$

whence

$$
\bar{Z}^{s} Z^{r}=\sum_{\substack{0 \leq p+q<<+s \\(p, q) \in I}} \overline{\alpha_{p q}} \bar{Z}^{q} Z^{p} .
$$

Thus, for $0 \leq k+\ell \leq n$,

$$
\begin{aligned}
\gamma_{s+k, r+\ell} & =\left\langle\bar{z}^{s} z^{r}, \bar{z}^{\ell} z^{k}\right\rangle_{M(n)} \\
& =\sum_{p, q} \overline{\alpha_{p q}}\left\langle\bar{z}^{q} z^{p}, \bar{z}^{\ell} z^{k}\right\rangle_{M(n)}=\sum_{p, q} \overline{\alpha_{p q}} \gamma_{q+k, p+\ell} .
\end{aligned}
$$

Now,

$$
\begin{aligned}
& \tau=\sum_{(k, \ell) \in I} a_{k \ell} \gamma_{k+s, \ell+r} \\
& =\sum_{(k, \ell) \in I} a_{k \ell} \sum_{\substack{0 \leq p+q<r+s \\
(p, q) \in I}} \overline{\alpha_{p q}} \gamma_{q+k, p+\ell} \\
& =\sum_{\substack{0 \leq p+q<r+s \\
(p, q) \in I}} \overline{\alpha_{p q}} \sum_{(k, \ell) \in I} a_{k \ell} \gamma_{q+k, p+\ell} \\
& =\sum_{\substack{0 \leq p+q<r+s \\
(p, q) \in I}} \overline{\alpha_{p q}}\left\langle\bar{z}^{i} z^{j}, \bar{z}^{p} z^{q}\right\rangle_{B} \\
& =\sum_{0 \leq p+q<r+s} \overline{\alpha_{p q}} \gamma_{i+q, j+p} \quad \text { (by case (i)); } \\
& (p, q) \in I
\end{aligned}
$$

thus

$$
\tau=\sum_{\substack{0 \leq p+q<r+s \\(p, q) \in I}} \overline{\alpha_{p q}} \gamma_{i+q, j+p} .
$$

Since $M(n)$ is recursively consistent, (2.15) implies

$$
\bar{Z}^{s+v} Z^{r+u}=\sum_{\substack{0 \leq p+q<r+s \\(p, q) \in I}} \overline{\alpha_{p q}} \bar{Z}^{q+v} Z^{p+u}
$$

whenever $u, v \geq 0$ satisfy $r+u+s+v=n+1$. Suppose $u$ and $v$ can additionally be chosen so that

$$
0 \leq u \leq j \text { and } 0 \leq v \leq i
$$


Let $L:=j-u, M:=i-v$; note that $L+M=(i+j)-(u+v)=n+1-(u+v) \leq n$, since $(r+s)+(u+v)=n+1$ implies $u+v \geq 1$. Now

$$
\begin{aligned}
\left\langle\bar{z}^{s+v} z^{r+u}, \bar{z}^{L} z^{M}\right\rangle_{B} & =\sum_{p, q} \overline{\alpha_{p q}}\left\langle\bar{z}^{q+v} z^{p+u}, \bar{z}^{L} z^{M}\right\rangle_{M(n)} \\
& =\sum_{p, q} \overline{\alpha_{p q}} \gamma_{q+v+M, p+u+L} \\
& =\sum_{p, q} \overline{\alpha_{p q}} \gamma_{q+i, p+j}=\tau \quad \text { (from (2.16)). }
\end{aligned}
$$

Define $h:=s-M$; since $r+s+u+v=i+j$, then $s-i+v=j-r-u$, whence $h=j-r-u$. Similarly, $s+v-h=i$ and $h+r=L$. Thus $\tau\left(=\left\langle\bar{z}^{s+v} z^{r+u}, \bar{z}^{L} z^{M}\right\rangle_{B}\right)$ normally generates

$$
\left\langle\bar{z}^{s+v-h} z^{r+u+h}, \bar{z}^{L-h} z^{M+h}\right\rangle_{B}=\left\langle\bar{z}^{i} z^{j}, \bar{z}^{r} z^{s}\right\rangle_{B}
$$

Thus, to complete the inductive definition of $\bar{Z}^{i} Z^{j}$, it remains to choose $u$ and $v$ satisfying (2.18). Let $P:=(n+1)-(r+s)(\geq 1)$. Since $M(n)$ is recursively consistent, the band of columns $Z^{r+P} \bar{Z}^{s}, \ldots, Z^{r} \bar{Z}^{s+P}$ is admissible; we distinguish whether $\bar{Z}^{i} Z^{j}$ (which is non-admissible) is to the left or right of this band within $B$.

(i) $\bar{Z}^{i} Z^{j}$ is to the left of the band; equivalently, $s>i$ and $j>r+P$. Let $u:=n+1-(r+s)(>0), v:=0$. Then $u+v+r+s=n+1$; since $i<s$, then $i<r+s$, whence $u=(n+1)-(r+s)=(i+j)-(r+s)<j$.

(ii) $\bar{Z}^{i} Z^{j}$ is to the right of the band; equivalently, $r>j$ and $i>s+P$. Let $u:=0, v:=(n+1)-(r+s)$. Since $r>j, v=i+j-(r+s)<i$.

Thus (2.13) is established. The completed column $\bar{Z}^{i} Z^{j}$ is consistent with moment matrix structure and satisfies $\bar{Z}^{i} Z^{j} \in \operatorname{Ran} M(n)$; thus, $\bar{Z}^{i} Z^{j}$ is added to $\mathcal{S}^{\prime}$, which completes the induction step.

Before we proceed with some applications of Theorem 2.7, we present a result which can help establish normal consistency for an extension block $B[n, n+1]$. This result shows that if $M(n)$ is recursively consistent, then each admissible column possesses a degree of "internal symmetry." Note that since $M(n)$ is recursively generated, then up to a symmetry, we can represent each admissible column as $\bar{Z}^{i} Z^{j+1}$, where $i+j=n$ and $\bar{Z}^{i} Z^{j}=p(Z, \bar{Z})$ for some $p \in \mathcal{P}_{n-1}$.

Proposition 2.9. Suppose $M(n)$ is recursively consistent, and suppose that $i+j=n, i \geq 1$, and $\bar{Z}^{i} Z^{j}=p(Z, \bar{Z})$ for some $p \in \mathcal{P}_{n-1}$. Then for $r+s=n$, $s \geq j$, and $r \geq i-1$, we have $\left\langle\bar{z}^{i} z^{j+1}, \bar{z}^{r} z^{s}\right\rangle_{B}=\overline{\left\langle\bar{z}^{i} z^{j+1}, \bar{z}^{s+i-j-1} z^{r+j+1-i}\right\rangle_{B}}$.

It is easy to see that the preceding identity holds in any moment matrix $M(n+1)$; this follows from normality and symmetry. Proof OF Proposition 2.9. Write $p(z, \bar{z}) \equiv \sum_{0 \leq k+\ell \leq n-1} a_{k \ell} \bar{z}^{k} z^{\ell} ;$ then $\bar{Z}^{i} Z^{j+1}=$
$\sum a_{k \ell} \bar{Z}^{k} Z^{\ell+1}$, so

$$
\begin{aligned}
\left\langle\bar{z}^{i} z^{j+1}, \bar{z}^{r} z^{s}\right\rangle_{B} & =\sum a_{k \ell}\left\langle\bar{z}^{k} z^{\ell+1}, \bar{z}^{r} z^{s}\right\rangle_{M(n)} \\
& =\sum a_{k \ell} \gamma_{k+s, \ell+1+r}
\end{aligned}
$$


Also,

$$
\begin{aligned}
\left\langle\bar{z}^{i} z^{j+1}, \bar{z}^{s+i-j-1} z^{r+j+1-i}\right\rangle_{B} & =\sum a_{k \ell}\left\langle\bar{z}^{k} z^{\ell+1}, \bar{z}^{s+i-j-1} z^{r+j+1-i}\right\rangle_{M(n)} \\
& =\sum a_{k \ell} \gamma_{k+r+j+1-i, \ell+s+i-j},
\end{aligned}
$$

so

$$
\overline{\left\langle\bar{z}^{i} z^{j+1}, \bar{z}^{s+i-j-1} z^{r+j+1-i}\right\rangle_{B}}=\sum \overline{a_{k \ell}} \gamma_{\ell+s+i-j, k+r+j+1-i} .
$$

Since $s \geq j, \ell+s+i-j=i+(\ell+s-j) \geq i$; since $r \geq i-1, k+r+j+1-i=$ $j+(k+r+1-i) \geq j$. Also, $\ell+s-j+k+r+1-i=(k+\ell+1)+(r+s)-(i+j) \leq n$; thus

$$
\begin{aligned}
\gamma_{i+(\ell+s-j), j+(k+r+1-i)} & =\left\langle\bar{z}^{i} z^{j}, \bar{z}^{k+r+1-i} z^{\ell+s-j}\right\rangle_{M(n)} \\
& =\sum_{0 \leq p+q \leq n-1} a_{p q}\left\langle\bar{z}^{p} z^{q}, \bar{z}^{k+r+1-i} z^{\ell+s-j}\right\rangle_{M(n)} \\
& =\sum a_{p q} \gamma_{p+\ell+s-j, q+k+r+1-i} .
\end{aligned}
$$

Then, from (2.19),

$$
\begin{aligned}
\overline{\left\langle\bar{z}^{i} z^{j+1}, \bar{z}^{s+i-j-1} z^{r+j+1-i}\right\rangle_{B}} & =\sum \overline{a_{k \ell}} \sum a_{p q} \gamma_{p+\ell+s-j, q+k+r+1-i} \\
& =\sum a_{p q} \sum \overline{a_{k \ell}} \gamma_{p+\ell+s-j, q+k+r+1-i} \\
& =\sum a_{p q} \sum \overline{a_{k \ell}} \gamma_{\ell+(p+s-j), k+(q+r+1-i)} \\
& =\sum a_{p q} \gamma_{p+s, q+r+1}
\end{aligned}
$$

(since $\bar{Z}^{j} Z^{i}=\sum \overline{a_{k \ell}} \bar{Z}^{\ell} Z^{k}$ and $\gamma_{j+x, i+y}=\sum \overline{a_{k \ell}} \gamma_{\ell+x, k+y}$ )

$$
\begin{aligned}
& =\sum a_{p q}\left\langle\bar{z}^{p} z^{q+1}, \bar{z}^{r} z^{s}\right\rangle_{M(n)} \\
& =\left\langle\bar{z}^{i} z^{j+1}, \bar{z}^{r} z^{s}\right\rangle_{B} .
\end{aligned}
$$

The next two examples illustrate Proposition 2.9.

EXAMPLE 2.10. Suppose $M(4) \geq 0$ is recursively consistent and $\bar{Z} Z^{3}=p(Z, \bar{Z})$ for some $p \in \mathcal{P}_{3}$. Recursively generate $\bar{Z} Z^{4}:=(z p)(Z, \bar{Z})$, and denote this vector by $(x, y, w, u, v)^{t}$. Apply Proposition 2.9 with $n=4, i=1, j=3, r=0$, and $s=4$ (observe that $s>j$ and $r=i-1$ ). Then

$$
x=\left\langle\bar{z} z^{4}, z^{4}\right\rangle_{B}=\overline{\left\langle\bar{z} z^{4}, \bar{z} z^{3}\right\rangle_{B}}=\bar{y} .
$$

We may also recursively generate $Z^{3} \bar{Z}^{2}=(\bar{z} p)(Z, \bar{Z})$; by Proposition $2.3, Z^{4} \bar{Z}$ and $Z^{3} \bar{Z}^{2}$ form a normal band. Similarly, $\bar{Z}^{4} Z$ and $\bar{Z}^{3} Z^{2}$ form a normal band generated from the relation

$$
\bar{Z}^{3} Z=\bar{p}(Z, \bar{Z})
$$


Thus, the proposed $B_{n, n+1}$ block of any flat extension of $M(4)$ must be of the form

$$
\left(\begin{array}{cccccc}
Z^{5} & \bar{Z} Z^{4} & \bar{Z}^{2} Z^{3} & \bar{Z}^{3} Z^{2} & \bar{Z}^{4} Z & \bar{Z}^{5} \\
* & x & z & \bar{u} & \bar{v} & * \\
* & \bar{x} & x & \bar{w} & \bar{u} & * \\
* & w & \bar{x} & x & \bar{w} & * \\
* & u & w & \bar{x} & x & * \\
* & v & u & \bar{z} & \bar{x} & *
\end{array}\right) .
$$

Thus $Z^{4} \bar{Z}, Z^{3} \bar{Z}^{2}, Z^{2} \bar{Z}^{3}, Z \bar{Z}^{4}$ form a normal band if and only if $z=\bar{w}$. Example 2.5 shows that in general, $z \neq \bar{w}$, and that there need not be a positive extension.

EXAMPle 2.11. Suppose $M(3)$ is recursively consistent and suppose $Z^{3}=$ $p(Z, \bar{Z})$ for some $p \in \mathcal{P}_{2}$. We generate $Z^{3} \bar{Z} \equiv(\bar{z} p)(Z, \bar{Z})$ and claim that $\left\langle\bar{z} z^{3}, z^{3}\right\rangle_{B}=$ $\overline{\left\langle\bar{z} z^{3}, \bar{z} z^{2}\right\rangle_{B}}$. Indeed, we have $\bar{Z}^{3}=\bar{p}(Z, \bar{Z})$ and $Z \bar{Z}^{3}=(z \bar{p})(Z, \bar{Z})$. Apply Proposition 2.9 with $n=3, i=3, j=0, r=2$, and $s=1$ to conclude that $\left\langle\bar{z}^{3} z, \bar{z}^{2} z\right\rangle_{B}=\overline{\left\langle\bar{z}^{3} z, \bar{z}^{3}\right\rangle_{B}}$; now apply symmetry in block $B$ (Proposition 2.4).

Our first application of the preceding results on consistency is a new proof of the fact that a positive flat moment matrix $M(n)$ has a unique flat extension $M(n+1)$.

Proposition 2.12. [CuF4, Theorem 5.4] If $M(n) \geq 0$ and $\operatorname{rank} M(n)=$ rank $M(n-1)$, then $M(n)$ has a unique flat extension $M(n+1)$.

Proof. Theorem 1.6 implies that $M(n-1)$ is recursively generated. Since, by hypothesis, $M(n)$ is a flat extension of $M(n-1)$, Proposition 1.7 implies that $M(n)$ is recursively generated. Further, $[\mathbf{C u F} 4$, Lemma 5.9] implies that $M(n)$ is recursively consistent. For each $i+j=n$, we have $\bar{Z}^{i} Z^{j}=p_{i j}(Z, \bar{Z})$ with $p_{i j} \in \mathcal{P}_{n-1}$. Thus $\bar{Z}^{i} Z^{j+1} \equiv\left(z p_{i j}\right)(Z, \bar{Z})$ and $\bar{Z}^{i+1} Z^{j} \equiv\left(\bar{z} p_{i j}\right)(Z, \bar{Z})$ are admissible, and are normal with respect to each other by Proposition 2.3. Since $\left(\bar{z} p_{i j}\right)(Z, \bar{Z})=\left(z p_{i+1, j-1}\right)(Z, \bar{Z})$, it follows that the $B$ block is normal, and Proposition 2.4 readily implies that $B$ is symmetric. Since $\operatorname{Ran} B \subseteq \operatorname{Ran} M(n)$, it now follows from Proposition 2.6 that the $C$ block of $M \equiv[M(n) ; B]$ is Toeplitz, so $M$ is the unique flat extension of $M(n)$ of the form $M(n+1)$.

We next apply Theorem 2.7 to a flat extension problem for $M(3)$ in which $Z^{3}$ is recursively determined.

EXAMPle 2.13. Assume $M(3) \geq 0$ and $\mathcal{C}_{M(3)}$ has basis $I \equiv\left\{1, Z, \bar{Z}, Z^{2}, \bar{Z} Z\right.$, $\left.\bar{Z}^{2}, \bar{Z} Z^{2}, \bar{Z}^{2} Z\right\}$. Assume further that there exists $p \in \mathcal{P}_{2}$ such that $Z^{3}=p(Z, \bar{Z})$ (whence $\bar{Z}^{3}=\bar{p}(Z, \bar{Z})$ ). It follows readily that $M(3)$ is recursively generated and recursively consistent, with admissible columns $Z^{4}, \bar{Z} Z^{3}, \bar{Z}^{3} Z, \bar{Z}^{4}$. We claim that $M(3)$ is normally consistent. Proposition 2.3 implies that $Z^{4}, \bar{Z} Z^{3}$ and $\bar{Z}^{3} Z$, $\bar{Z}^{4}$ are each normal bands, so to establish normal consistency it suffices to verify $\left\langle\bar{z} z^{3}, \bar{z} z^{2}\right\rangle_{B}=\left\langle\bar{z}^{3} z, \bar{z}^{3}\right\rangle_{B}$ (since, by symmetry, this also implies $\left\langle\bar{z} z^{3}, z^{3}\right\rangle_{B}=$ $\left.\left\langle\bar{z}^{3} z, \bar{z}^{2} z\right\rangle_{B}\right)$. An application of Proposition 2.9 with $n=3, i=1, j=2, r=0$, $s=3$ shows that $\left\langle\bar{z} z^{3}, z^{3}\right\rangle_{B}=\overline{\left\langle\bar{z} z^{3}, \bar{z} z^{2}\right\rangle_{B}}$, and symmetry between $\bar{Z} Z^{3}$ and $\bar{Z}^{3} Z$ yields $\overline{\left\langle\bar{z} z^{3}, z^{3}\right\rangle_{B}}=\left\langle\bar{z}^{3} z, \bar{z}^{3}\right\rangle_{B}$; thus normality is established.

We now use normality to define $\bar{Z}^{2} Z^{2}$ via $\bar{Z}^{3} Z$ and $\bar{Z} Z^{3}$. Theorem 2.7 insures that the resulting block $B$ is a moment matrix block satisfying $\operatorname{Ran} B \subseteq \operatorname{Ran} M(3)$. From Proposition 2.6, we see that $\tilde{Z}^{4}$ and $\tilde{\bar{Z}} \tilde{Z}^{3}$ form a normal band in the $C$ block of $[M(3) ; B]$. Thus, using the reduced $C$-block test, $M(3)$ has a flat extension if and 
only if $C_{22}=C_{33}$; in this case the flat extension would be unique. To perform this test, let $\alpha:=M_{I}^{-1}\left[Z^{3} \bar{Z}\right]_{I}$ and let $\beta:=M_{I}^{-1}\left[\bar{Z}^{2} Z^{2}\right]_{I}$; then a flat extension exists if and only if

$$
\begin{aligned}
& \alpha_{1} \gamma_{31}+\alpha_{2} \gamma_{32}+\alpha_{3} \gamma_{41}+\alpha_{4} \gamma_{33}+\alpha_{5} \gamma_{42}+\alpha_{6} \gamma_{51}+\alpha_{7} \gamma_{43}+\alpha_{8} \gamma_{52} \\
& =\beta_{1} \gamma_{22}+\beta_{2} \gamma_{23}+\beta_{3} \gamma_{32}+\beta_{4} \gamma_{24}+\beta_{5} \gamma_{33}+\beta_{6} \gamma_{42}+\beta_{7} \gamma_{34}+\beta_{8} \gamma_{43} .
\end{aligned}
$$

To date, we have found no case in which (2.20) is valid.

Our next example illustrates a case in which (2.20) fails, so there is no flat extension.

EXAMPLE 2.14. We present an example of $M(3)$ positive, recursively generated, with $Z^{3}=\alpha \bar{Z}^{2}$, but with no flat extension $M(4)$. This shows that the hypothesis $k \leq\left[\frac{n}{2}\right]+1$ in Theorem 1.9 is necessary. (Here we have $n=3$, $k=3>2=\left[\frac{3}{2}\right]+1$.) Let $M(3)$ be given by

$$
\left(\begin{array}{cccccccccc}
1 & 0 & 0 & 0 & 1 & 0 & 0 & 0 & 0 & 0 \\
& 1 & & & & & 0 & \delta & & \\
& & 1 & & & & & 0 & \delta & 0 \\
0 & & & \delta & & & 0 & 0 & 0 & \bar{c} \\
1 & & & & \delta & & 0 & & & \\
0 & & & & & \delta & c & & & \\
& 0 & & 0 & 0 & \bar{c} & \frac{|c|^{2}}{\delta} & & & \\
& \delta & & 0 & & & & \frac{|c|^{2}}{\delta} & & \\
& 0 & \delta & 0 & & & & & \frac{|c|^{2}}{\delta} & \\
& 0 & & c & & & & & & \frac{|c|^{2}}{\delta}
\end{array}\right),
$$

where $\delta>1$ and $|c|>\delta^{3 / 2}$. Then $M(3)$ satisfies all the conditions of Example 2.13, with

$$
Z^{3}=\left(\frac{c}{\delta}\right) \bar{Z}^{2}
$$

If we further specify $\delta=4, c=9$, then (2.20) fails, so $M(3)$ has no flat extension. We shall see in Example 4.4 that $M(3)$ does not admit a representing measure.

We next begin an analysis of the flat extension problem for $M(3)$ in which $\bar{Z} Z^{2}$ is recursively determined.

EXAmple 2.15. Assume $M(3) \geq 0$ and $\mathcal{C}_{M(3)}$ has basis $\left\{1, Z, \bar{Z}, Z^{2}, \bar{Z} Z, \bar{Z}^{2}\right.$, $\left.Z^{3}, \bar{Z}^{3}\right\}$. Assume there exists $p \in \mathcal{P}_{2}$ such that $\bar{Z} Z^{2}=p(Z, \bar{Z})$ (whence $\bar{Z}^{2} Z=$ $\bar{p}(Z, \bar{Z})) . M(3)$ is recursively generated and we assume that $M(3)$ is recursively consistent, i.e., $(\bar{z} p)(Z, \bar{Z})=(z \bar{p})(Z, \bar{Z})$. The admissible columns are $\bar{Z} Z^{3}, \bar{Z}^{2} Z^{2}$, $\bar{Z}^{3} Z$, and it follows from Proposition 2.3 that these comprise a normal band; thus $M(3)$ is normally consistent. Theorem 2.7 permits us to define $Z^{4} \in \operatorname{Ran} M(3)$ (with a free choice for $\gamma_{07}$ ), and we define $\bar{Z}^{4}$ by symmetry. The resulting $B$ block is a moment matrix block with $\operatorname{Ran} B \subseteq \operatorname{Ran} M(3)$. Proposition 2.6 implies that in the $C$ block of $[M(3) ; B], \tilde{\bar{Z}} \tilde{Z}^{3}, \tilde{\bar{Z}}^{2} \tilde{Z}^{2}$ and $\tilde{\bar{Z}}^{3} \tilde{Z}$ are normal with respect to each other. Thus, by the reduced $C$-block test, $M(3)$ has a flat extension if and only if $C_{11}=C_{22}$ for some choice of $\gamma_{07}$. In Example 4.8 we illustrate a case in which a flat extension does exist and we explicitly compute the corresponding representing measure. 
We conclude this chapter with a reduction in the flat extension problem for $M(2)$.

ExAmple 2.16. Assume $M(2) \geq 0$ and $\mathcal{C}_{M(2)}$ has basis $\left\{1, Z, \bar{Z}, Z^{2}, \bar{Z}^{2}\right\}$. Assume there exists $p \in \mathcal{P}_{1}$ such that $\bar{Z} Z=p(Z, \bar{Z})$. Then $M(2)$ is recursively generated and is recursively consistent (with $\bar{Z} Z^{2}$ and $\bar{Z}^{2} Z$ admissible). Proposition 2.3 implies that $M(2)$ is normally consistent. Theorem 2.7 implies that we may define $Z^{3} \in \operatorname{Ran} M(2)$ (in moment matrix form, with $\gamma_{05}$ free), and we may then define $\bar{Z}^{3}$ by symmetry. It follows from the reduced $C$-block test that $M(2)$ has a flat extension if and only if there is some $\gamma_{05}$ for which $C_{11}=C_{22}$ in the $C$ block of $[M(2) ; B]$. We pursue this question in more detail in Chapter 3 , and obtain positive results in certain cases. 


\section{CHAPTER 3}

\section{The Singular Quartic Moment Problem}

We devote this chapter to the proof of Theorem 1.10; thus, we will study in detail the truncated moment problem for $n=2$, with an associated $6 \times 6$ moment matrix $M(2)$ which is singular. Henceforth, we assume that $M(2)$ is positive and recursively generated. If $M(1)$ is singular, then, without loss of generality, $\bar{Z} \in\langle 1, Z\rangle$ ([CuF4, Lemma 3.10] and [Fia1, Proposition 2.4]), so Theorem 1.8 implies that $M(1)$ has a flat extension; we may thus assume that $\operatorname{det} M(1)>0$, or, equivalently, that $\{1, Z, \bar{Z}\}$ is linearly independent. If $Z^{2} \in\langle 1, Z, \bar{Z}\rangle$, then it follows from Theorem 1.9 (with $n=k=2$ ) that $M(2)$ has a unique flat extension; we may thus assume in the sequel that $\left\{1, Z, \bar{Z}, Z^{2}\right\}$ is linearly independent. The remainder of this chapter is devoted to analyzing the case $\bar{Z} Z \in\langle 1, Z, \bar{Z}\rangle$.

Consider the column $\bar{Z} Z$, and assume that

$$
\bar{Z} Z=c_{1} 1+c_{2} Z+c_{3} \bar{Z} .
$$

By [CuF4, Lemma 3.10], (3.1) gives rise to

$$
\bar{Z} Z=\bar{c}_{1} 1+\bar{c}_{3} Z+\bar{c}_{2} \bar{Z}
$$

and therefore $c_{1} \in \mathbb{R}$ and $c_{3}=\bar{c}_{2}$. Let us make some preliminary observations concerning $\mathcal{V}(\gamma)$ (defined in (1.6)). Let $p(z, \bar{z}):=z \bar{z}-c_{1}-c_{2} z-\bar{c}_{2} \bar{z}$. Then $z \equiv x+i y \in \mathcal{Z}(p)$ if and only if $\left|z-\bar{c}_{2}\right|^{2}=c_{1}+\left|c_{2}\right|^{2}$. If $c_{1}+\left|c_{2}\right|^{2} \leq 0$, then $\rho(\gamma)<0$ by (1.7). We may thus assume that $c_{1}+\left|c_{2}\right|^{2}>0$.

Our approach to the singular quartic moment problem with $\left\{1, Z, \bar{Z}, Z^{2}\right\}$ independent and $M(2)$ subject to (3.1) is divided into two aspects: (i) conditions on the data that guarantee the positivity of $M(2)$, and (ii) the actual search for flat moment matrix extensions, assuming $M(2) \geq 0$. To simplify somewhat our calculations, we shall focus attention primarily on finite collections $\gamma: \gamma_{00}, \ldots, \gamma_{40}$ such that $\gamma_{i j} \in \mathbb{R}$ for $0 \leq i+j \leq 3$, that is,

$$
M(2) \equiv\left(\begin{array}{ccc|ccc}
a & b & b & c & d & c \\
b & d & c & e & e & f \\
b & c & d & f & e & e \\
\hline c & e & f & g & h_{1}-i h_{2} & u-i v \\
d & e & e & h_{1}+i h_{2} & g & h_{1}-i h_{2} \\
c & f & e & u+i v & h_{1}+i h_{2} & g
\end{array}\right) .
$$

We first analyze conditions on $a, b, c, d, e, f$ that ensure the positivity of $M(2)$. For $A$ a positive $N \times N$ matrix, let us denote by $[A]_{k}$ the compression of $A$ to the first $k$ rows and columns; similarly, the first $k$ entries of a column $C$ will be denoted by $[C]_{k}$. More generally, if $1 \leq n_{1}<\cdots<n_{k} \leq N$ we let $[A]_{\left\{n_{1}, \ldots, n_{k}\right\}}$ denote the compression of $A$ to the rows and columns indexed by $\left\{n_{1}, \ldots, n_{k}\right\}$. We begin with a characterization of $[M(2)]_{4}>0$ in terms of the matrix entries. 
Proposition 3.1. $[M(2)]_{4}$ is positive and invertible if and only if $a>0, d>c$,

$$
a(c+d)>2 b^{2}
$$

and

$$
g>\left(\begin{array}{lll}
c & e & f
\end{array}\right) M(1)^{-1}\left(\begin{array}{lll}
c & e & f
\end{array}\right)^{T} .
$$

Proof. By the Nested Determinant Test and Choleski's Algorithm, $[M(2)]_{4}$ is positive and invertible if and only if $\operatorname{det}[M(2)]_{1}>0, \operatorname{det}[M(2)]_{2}>0, \operatorname{det}[M(2)]_{3}>$ 0 , and $\operatorname{det}[M(2)]_{4}>0$, that is, $a>0$,

$$
\begin{gathered}
a d-b^{2}>0 \\
d>\left(\begin{array}{ll}
b & c
\end{array}\right)\left(\begin{array}{ll}
a & b \\
b & d
\end{array}\right)^{-1}\left(\begin{array}{l}
b \\
c
\end{array}\right)
\end{gathered}
$$

and $g>\left(\begin{array}{lll}c & e & f\end{array}\right) M(1)^{-1}\left(\begin{array}{lll}c & e & f\end{array}\right)^{T}$. A calculation shows that if we assume (3.5), then (3.6) holds if and only if $d>c$ and $a(c+d)-2 b^{2}>0$. Conversely, if $d>c$ and (3.3) holds, then (3.5) holds, whence (3.6) holds.

Due to (3.1), $M(2)$ is a flat extension of $[M(2)]_{\{1,2,3,4,6\}}$. Thus, the positivity of $M(2)$ is equivalent to that of $[M(2)]_{\{1,2,3,4,6\}}$ (by Proposition 2.1), which, under the assumption $[M(2)]_{4}>0$, is equivalent to $\operatorname{det}[M(2)]_{\{1,2,3,4,6\}} \geq 0$ (again, by the Nested Determinant Test). To study this condition, we first obtain a result which describes some intrinsic structure present in $M(2)$.

Proposition 3.2. Assume $M(2)$ satisfies (3.1), with $M(1)$ invertible. Then $g$ and $h$ can be written in terms of $a, b, c, d, e, f$, namely

$$
g=\left(\begin{array}{lll}
d & e & e
\end{array}\right) M(1)^{-1}\left(\begin{array}{lll}
d & e & e
\end{array}\right)^{T}
$$

and

$$
h=\left(\begin{array}{lll}
c & f & e
\end{array}\right) M(1)^{-1}\left(\begin{array}{lll}
d & e & e
\end{array}\right)^{T} .
$$

As a consequence, $h$ must also be real.

Proof. We know that $[\bar{Z} Z]_{3}=c_{1}[1]_{3}+c_{2}[Z]_{3}+\bar{c}_{2}[\bar{Z}]_{3}$, and since $M(1)$ is invertible, we must have $\left(\begin{array}{lll}c_{1} & c_{2} & \bar{c}_{2}\end{array}\right)^{T}=M(1)^{-1}\left(\begin{array}{lll}d & e & e\end{array}\right)^{T}$. Observe now that $g$ and $h$ are the fifth and sixth entries of $\bar{Z} Z$, which must also obey (3.1). The result is now obvious. forces

Returning now to the positivity of $[M(2)]_{\{1,2,3,4,6\}}$, (3.7) together with $(3.4)$

$$
\left(\begin{array}{lll}
d & e & e
\end{array}\right) M(1)^{-1}\left(\begin{array}{lll}
d & e & e
\end{array}\right)^{T}>\left(\begin{array}{lll}
c & e & f
\end{array}\right) M(1)^{-1}\left(\begin{array}{lll}
c & e & f
\end{array}\right)^{T},
$$

which gives rise to a quadratic form in $f$, whose discriminant is positive if and only if

$$
-2 b^{2} c d+a c^{2} d-b^{2} d^{2}+2 a c d^{2}+a d^{3}+4 b^{3} e-2 a b c e-4 a b d e+a^{2} e^{2}>0 .
$$

This expression in turn is a quadratic form in $e$, whose discriminant is given by $4\left(b^{2}-a d\right)\left(-2 b^{2}+a(c+d)\right)^{2}$, which is negative by $(3.5)$; since the coefficient of $e^{2}$ is positive, (3.10) will hold (for every real value of $e$ !) if and only if $F(a, b, c, d):=-2 b^{2} c d+a c^{2} d-b^{2} d^{2}+2 a c d^{2}+a d^{3}>0$. Observe, however, that $F(a, b, c, d)=d\left\{\left[a(c+d)-2 b^{2}\right](c+d)+b^{2} d\right\}$, which is positive by (3.3). We thus arrive at the following positivity criterion for $[M(2)]_{4}$, solely in terms of $M(1)$. 
Proposition 3.3. Assume that the $\bar{Z} Z$ column of $M(2)$ is given by (3.1) and that $M(1)$ is positive and invertible. Then for every choice of $e$ and for $g$ given by (3.7), there exists an open interval of values of $f$ which make $[M(2)]_{4}$ positive and invertible.

We can now obtain a criterion for the positivity of $[M(2)]_{\{1,2,3,4,6\}}$.

Proposition 3.4. Assume that values for $a, b, c, d$, e and $f$ have been chosen so that $M(2)$ satisfies (3.1) and $[M(2)]_{4}>0$, with $g$ and $h$ given by (3.7) and (3.8), respectively. Then $[M(2)]_{\{1,2,3,4,6\}} \geq 0$ if and only if $u+i v$ belongs to the closed disc with center

$$
c_{0}:=\left(\begin{array}{lll}
c & f & e
\end{array}\right) M(1)^{-1}\left(\begin{array}{lll}
c & e & f
\end{array}\right)^{T}
$$

and radius

$$
r:=\left(\begin{array}{lll}
d & e & e
\end{array}\right) M(1)^{-1}\left(\begin{array}{lll}
d & e & e
\end{array}\right)^{T}-\left(\begin{array}{lll}
c & e & f
\end{array}\right) M(1)^{-1}\left(\begin{array}{lll}
c & e & f
\end{array}\right)^{T} .
$$

Moreover,

$$
\operatorname{rank}[M(2)]_{\{1,2,3,4,6\}}=4 \Longleftrightarrow\left|u+i v-c_{0}\right|=r
$$

and

$$
\operatorname{rank}[M(2)]_{\{1,2,3,4,6\}}=5 \Longleftrightarrow\left|u+i v-c_{0}\right|<r .
$$

Proof. Observe that $[M(2)]_{\{1,2,3,4,6\}}=\left(\begin{array}{cc}M(1) & B \\ B^{*} & C\end{array}\right)$, where $B:=\left(\begin{array}{lll}c & e & f \\ c & f & e\end{array}\right)^{T}$ and $C:=\left(\begin{array}{cc}g & u-i v \\ u+i v & g\end{array}\right)$. By the result of Smul'jan discussed in the paragraph preceding (2.4), the positivity of $[M(2)]_{\{1,2,3,4,6\}}$ is equivalent to the matrix inequality



$$
\begin{aligned}
& =C-B^{*} M(1)^{-1} B \geq 0 .
\end{aligned}
$$

Since the $(1,1)$-entry of the above matrix is positive by $(3.9)$, and since a direct calculation shows that the $(2,2)$-entry equals the $(1,1)$-entry, the result now follows via Choleski's Algorithm.

To summarize, we can build moment matrices $M(2) \geq 0$ satisfying (3.1) and $[M(2)]_{4}>0$ as follows.

\section{Algorithm 3.5.}

(i) Choose $a>0, b$ arbitrary, and $c$ arbitrary;

(ii) Select $d>\max \left\{c, \frac{2 b^{2}}{a}-c\right\}$;

(iii) Pick $e$ arbitrary and define $g$ by (3.7);

(iv) Choose $f$ in the positivity interval of (3.9);

(v) Define $h$ by $(3.8)$;

(vi) Finally, select $u+i v$ according to Proposition 3.4 (to make $\operatorname{rank} M(2)=4$ or $\operatorname{rank} M(2)=5)$.

ExAmPle 3.6. Let $a=1, b=0, c=-1$. Then $d$ must be chosen bigger than 1 , say for instance $d=2$. Let $e:=-2$; by $(3.7), g=12$. In this case, the positivity interval for $(3.9)$ is $\left[\frac{1-3 \sqrt{6}}{2}, \frac{1+3 \sqrt{6}}{2}\right]$; choose, for instance, $f:=1$. By $(3.8), h=0$. It then follows that $c_{0}=0$ and $r=9$. To make rank $M(2)=4$ take for instance $u:=0, v:=9 ;$ to have $\operatorname{rank} M(2)=5$, let $u:=0, v:=0$. 
We now turn our attention to the problem of building a flat moment matrix extension $M(3)$ of $M(2)$, given as just described. First, observe that $M(2)$ is clearly recursively generated. Since the only recursively determined column is $\bar{Z} Z$, it is also clear that $M(2)$ is recursively consistent, and Proposition 2.3 implies that $M(2)$ is normally consistent. We also notice that the relation (3.1) must induce in a proposed flat extension $M(3)$ the relation

$$
\bar{Z} Z^{2}=c_{1} Z+c_{2} Z^{2}+\bar{c}_{2} \bar{Z} Z
$$

so the entries $\gamma_{23}$ and $\gamma_{14}$ are fully determined, namely,

$$
\gamma_{23}=c_{1} e+c_{2} h+\bar{c}_{2} g
$$

and

$$
\gamma_{14}=c_{1} f+c_{2}(u+i v)+\bar{c}_{2} h .
$$

It follows that in any proposed flat extension $M(3)$, the $Z^{3}$ column must satisfy the condition $\left[Z^{3}\right]_{6}=\left(\begin{array}{llllll}\gamma_{03} & \gamma_{13} & \gamma_{04} & \gamma_{23} & \gamma_{14} & \gamma_{05}\end{array}\right)$, where $\gamma_{23}$ and $\gamma_{14}$ are given by (3.12) and (3.13), respectively, and where $\gamma_{05}$ is not yet determined. Moreover, $\left[Z^{3}\right]_{6}$ must be in the range of $M(2)$, and since $[M(2)]_{4}$ is invertible, two cases arise, which we consider separately.

1. $\operatorname{rank} M(2)=4$. (Proof of Theorem $1.10(\mathrm{ii})$ ) The basis for $\mathcal{C}_{M(2)}$ is $\left\{1, Z, \bar{Z}, Z^{2}\right\}$, and observe that this implies that $\bar{Z}^{2} \notin\{1, Z, \bar{Z}\}$, for otherwise $Z^{2} \in\{1, Z, \bar{Z}\}$ by $[\mathbf{C u F} 4$, Proposition 3.10]. Thus, $M(2)$ does not satisfy the hypotheses of Theorem 2.7 ; in this case we will prove the existence of flat extensions by an independent but related argument. To define $Z^{3}$ so that it is normal with respect to $\bar{Z} Z^{2}$ we must have $\left[Z^{3}\right]_{4}=\mathbf{v}:=\left(\begin{array}{llll}f & h & u+i v & \gamma_{23}\end{array}\right)^{T}$, with $\gamma_{23}$ given by (3.12). Since $[M(2)]_{4}>0$, there exist unique scalars $k_{1}, k_{2}, k_{3}, k_{4}$ such that $[M(2)]_{4}\left(\begin{array}{llll}k_{1} & k_{2} & k_{3} & k_{4}\end{array}\right)^{T}=\mathbf{v}$. To ensure that $Z^{3} \in \operatorname{Ran} M(2)$, we are thus forced to define $Z^{3}:=M(2) \cdot\left(\begin{array}{llllll}k_{1} & k_{2} & k_{3} & k_{4} & 0 & 0\end{array}\right)^{T}$. Note that this relation defines $\gamma_{05}$, and "redefines" $\gamma_{14}$. To ensure normality, the fifth entry of $\left[Z^{3}\right]_{6}$ and the sixth entry of $\left[\bar{Z} Z^{2}\right]_{6}$ must coincide, i.e.,

$$
\left(\begin{array}{llll}
d & e & e & h
\end{array}\right)\left(\begin{array}{llll}
k_{1} & k_{2} & k_{3} & k_{4}
\end{array}\right)^{T}=\gamma_{14} .
$$

Using (3.13) and symbolic manipulation, we verify (3.14), a fact that can also be derived from the proof of Theorem 2.7 (see Example 2.16). As for the rest of the $B$ block of a proposed $M(3)$, we observe that $\bar{Z}^{2} Z:=c_{1} \bar{Z}+\bar{c}_{2} \bar{Z}^{2}+c_{2} \bar{Z} Z$ is normal with respect to $\bar{Z} Z^{2}$ (by Proposition 2.3). Finally, we define $\bar{Z}^{3}$ by symmetry, $\bar{Z}^{3}:=$ $\bar{k}_{1} 1+\bar{k}_{2} \bar{Z}+\bar{k}_{3} Z+\bar{k}_{4} \bar{Z}^{2}$. Since $\left\{Z^{3}, \bar{Z} Z^{2}\right\}$ and $\left\{\bar{Z} Z^{2}, \bar{Z}^{2} Z\right\}$ form normal bands, and since $\bar{Z}^{2} Z, \bar{Z}^{3}$ are symmetric with respect to $Z^{3}, \bar{Z} Z^{2}$, respectively, we can conclude that $B$ is symmetric and satisfies normality. Thus, we have constructed a moment matrix extension block $B$ satisfying $\operatorname{Ran} B \subseteq \operatorname{Ran} M(2)$. The existence of a flat extension now depends on the reduced $C$-test, that is, on whether the $(1,1)$ and $(2,2)$ entries of the $C$ block of $[M(2) ; B]$ are equal. Our next result shows that this is indeed the case.

Theorem 3.7. Let $M(2)$ satisfy (3.1), assume $M(2) \geq 0,[M(2)]_{4}>0$ and $\operatorname{rank} M(2)=4$ (as given by Algorithm 3.5). Then there exists a unique flat extension $M(3)$ of $M(2)$. Consequently, there is a unique 4-atomic representing measure for $\gamma$. 
Proof. The reduced $C$-test consists of verifying that

$$
\left(\begin{array}{llll}
\gamma_{30} & \gamma_{31} & \gamma_{40} & \gamma_{32}
\end{array}\right)\left(\begin{array}{lllll}
k_{1} & k_{2} & k_{3} & k_{4}
\end{array}\right)^{T}=\left(\begin{array}{llll}
\gamma_{22} & \gamma_{23} & \gamma_{32}
\end{array}\right)\left(\begin{array}{lll}
c_{1} & c_{2} & \bar{c}_{2}
\end{array}\right)^{T},
$$

that is

$$
\begin{aligned}
& k_{1} f+k_{2} h+k_{3} j+k_{4}\left(c_{1} e+c_{2} h+\bar{c}_{2} g\right)-c_{1} g \\
&+ c_{2}\left(c_{1} e+c_{2} h+\bar{c}_{2} g\right)+\bar{c}_{2}\left(c_{1} e+c_{2} h+\bar{c}_{2} g\right)=0 .
\end{aligned}
$$

Using symbolic manipulation, one shows that (3.15) is equivalent to

$$
-\frac{1+e^{2}}{1+e^{2}-f^{2}} \cdot \operatorname{det} M(2)_{\{1,2,3,4,6\}}=0,
$$

which follows from the fact that $\operatorname{rank} M(2)=4$. (Incidentally, notice that $\gamma_{05}$ played no role in (3.15).)

ExAmple 3.6 (CONTinued). Recall that $a=1, b=0, c=-1, d=2, e=-2$, $f=1, g=12$, and $h=0$; to make $\operatorname{rank} M(2)=4$, choose $u=0$ and $v=9$. In (3.1), $c_{1}=2, c_{2}=c_{3}=-2$, and by (3.12) and (3.13) we have $\gamma_{23}=-28$ and $\gamma_{14}=2-18 i$. Also, $k_{1}=-2, k_{2}=-3+3 i, k_{3}=6 i$, and $k_{4}=-3$, from which it follows that $\gamma_{05}=-1+36 i$ and $\gamma_{33}=136$. Since $M(3)$ (as defined by Theorem 3.7) is flat, Theorem 1.1 implies that $M(3)$ has a unique flat extension $M(4)$, which is recursively generated by Proposition 1.7. To obtain the unique representing measure $\mu$ guaranteed by Theorem 3.7, we proceed as follows. (3.1) induces (3.11) and, a fortiori,

$$
\bar{Z} Z^{3}=c_{1} Z^{2}+c_{2} Z^{3}+\bar{c}_{2} \bar{Z} Z^{2}=2 Z^{2}-2 Z^{3}-2 \bar{Z} Z^{2},
$$

this time in $\mathcal{C}_{M(4)}$. (3.16) yields at once $\gamma_{24}=52+36 i$ and $\gamma_{34}=-432-$ $72 i$. We now look at the "analytic" compression of $M(3)$, that is $[M(3)]_{\text {anal }}:=$ $[M(3)]_{\{1,2,4,7\}}$. Observe that $[M(3)]_{\text {anal }}$ is positive and invertible: for, if $Z^{3} \in$ $\left\langle 1, Z, Z^{2}\right\rangle$ in $\mathcal{C}_{[M(3)]_{\text {anal }}}$ then the Extension Principle would force the same linear dependence in $\mathcal{C}_{M(3)}$, which would immediately place an upper bound of 3 on the number of atoms of any representing measure (by (1.4)), contradicting Theorem 3.7. We now use the invertibility of $[M(3)]_{\text {anal }}$ to express $\left[Z^{4}\right]_{\text {anal }}$ in terms of $[1]_{\text {anal }}$, $[Z]_{\text {anal }},\left[Z^{2}\right]_{\text {anal }},\left[Z^{3}\right]_{\text {anal }}$ in $\mathcal{C}_{[M(3)]_{\text {anal }}}$. By the Extension Principle, this relation extends to the corresponding relation in $\mathcal{C}_{M(4)}$, and thus gives rise to the polynomial equation

$$
z^{4}+5 z^{3}+(9-3 i) z^{2}+(8+6 i) z+(4-12 i)=0,
$$

which is satisfied by every point in the support of $\mu$, by (1.4). The roots of (3.17) are $z_{0}=\frac{-1+3 i-(-1)^{3 / 4} \sqrt{6}}{2}, z_{1}=\frac{-1+3 i+(-1)^{3 / 4} \sqrt{6}}{2}, z_{2}=\frac{-4-3 i-\sqrt{15}}{2}$ and $z_{3}=\frac{-4-3 i+\sqrt{15}}{2}$, which thus become the atoms of $\mu$. To find the densities, we form the associated $4 \times 4$ Vandermonde system (1.3), and obtain $\rho_{0} \cong 0.554371, \rho_{1} \cong 0.0919931$, $\rho_{2} \cong 0.0175098$ and $\rho_{3} \cong 0.346127$.

Proof of Theorem 1.10(iii). The preceding rank 4 case can be generalized to permit complex entries for the moments in $M(2)$. We do not have an analogue of Algorithm 3.5 to describe the most general $M(2) \geq 0$ for which $\left\{1, Z, \bar{Z}, Z^{2}\right\}$ is a basis for $\mathcal{C}_{M(2)}$ and $\bar{Z} Z \in\langle 1, Z, \bar{Z}\rangle$; nevertheless, we may proceed exactly as before to reduce the question of flat extensions to the reduced $C$-block test $C_{11}=C_{22}$.

We conjecture that the latter test is always satisfied. 
2. $\operatorname{rank} M(2)=5$. (Proof of TheOrem $1.10(\mathrm{iv}))$ This is a continuation of Example 2.16, so we permit the moments in $M(2)$ to be complex. Recall from Example 2.16 that to each $\gamma_{05} \in \mathbb{C}$ there corresponds a unique moment matrix extension block $B \equiv B\left[\gamma_{05}\right]$ such that $\operatorname{Ran} B \subseteq \operatorname{Ran} M(2)$. Thus, $M(2)$ admits a flat extension $M(3)$ if and only if we can choose $\gamma_{05}$ so that the reduced $C$-block test $C_{11}=C_{22}$ is satisfied.

The preceding test now assumes the form

$$
\begin{aligned}
\left(\begin{array}{lllll}
\gamma_{30} & \gamma_{31} & \gamma_{40} & \gamma_{32} & \gamma_{50}
\end{array}\right)\left(M(2)_{\{1,2,3,4,6\}}\right)^{-1}\left(\begin{array}{lllll}
\gamma_{03} & \gamma_{13} & \gamma_{04} & \gamma_{23} & \gamma_{05}
\end{array}\right)^{T} \\
=\left(\begin{array}{lllll}
\gamma_{22} & \gamma_{23} & \gamma_{32}
\end{array}\right)\left(\begin{array}{llll}
c_{1} & c_{2} & \bar{c}_{2}
\end{array}\right)^{T}
\end{aligned}
$$

where $\gamma_{23}$ is given as in (3.12). At present, we have not been able to verify (3.18) in general; however, in a number of cases, two of which we next illustrate, we can establish the existence of flat extensions.

EXAMPLE 3.8. Set $a=1, b=0, c=0$, and $d=1$ in $(3.2)$. Then $\operatorname{det} M(2)_{4}=$ $1+e^{2}-f^{2}$, which imposes the condition $|f|<\sqrt{1+e^{2}}$. To simplify the calculations, we set $f:=\sqrt{1+e^{2}-r}$, for $0<r<1+e^{2}$. Then $\operatorname{det} M(2)_{4}=r$ and

$$
\operatorname{det} M(2)_{\{1,2,3,4,6\}}=\operatorname{det}\left(\begin{array}{cc}
r & u-i v-2 e f \\
u+i v-2 e f & r
\end{array}\right) \text {. }
$$

Since det $M(2)_{\{1,2,3,4,6\}}$ must be positive, for simplicity we assign to $u+i v$ the value $2 e f$; then $\operatorname{det} M(2)_{\{1,2,3,4,6\}}=r^{2}, g=1+2 e^{2}$, and $h=e^{2}+e f$. Since (3.1) holds with $c_{1}=1$ and $c_{2}=e$, we obtain $\gamma_{14}=e^{3}+f+3 e^{2} f$ and $\gamma_{23}=e\left(2+3 e^{2}+e f\right)$. With $\gamma_{05} \equiv x \in \mathbb{R}$, the $C$-block test reduces to finding at least one value of $x$ satisfying the equation

$$
\begin{aligned}
-1-3 e^{2}-3 e^{4}-e^{6}+2 f^{2}+ & 4 e^{2} f^{2}+11 e^{4} f^{2} \\
& +6 e^{3} f^{3}-f^{4}+\left(-6 e^{2} f-2 e f^{2}\right) x+x^{2}=0 .
\end{aligned}
$$

Observe that the discriminant of (3.19) is $4 r^{2}\left(1+e^{2}\right)$, which shows at once that there are two values of $x$ which fulfill the test. Thus, $M(2)$ does admit a flat extension $M(3)$ in this case (and the corresponding representing measure is 5-atomic).

Proposition 3.9. In (3.2), assume $a=1, b=0, c=0, d=1$ and $f=0$, that is

$$
M(2) \equiv\left(\begin{array}{ccc|ccc}
1 & 0 & 0 & 0 & 1 & 0 \\
0 & 1 & 0 & e & e & 0 \\
0 & 0 & 1 & 0 & e & e \\
\hline 0 & e & 0 & 1+2 e^{2} & e^{2} & u-i v \\
1 & e & e & e^{2} & 1+2 e^{2} & e^{2} \\
0 & 0 & e & u+i v & e^{2} & 1+2 e^{2}
\end{array}\right)
$$

Assume also that $\operatorname{rank} M(2)=5$, that is $u^{2}+v^{2}<\left(1+e^{2}\right)^{2}$ (cf. Proposition 3.4). Then, for every choice of $u$ and $v$ there exist real numbers $r$ and $s$ such that with $\gamma_{05}:=r+i s$, the reduced $C$-block test is satisfied. In other words, $M(2)$ does admit a flat extension $M(3)$. 
Proof. Direct calculation shows that in this case the reduced $C$-block test is equivalent to

$$
\begin{aligned}
F(r, s):= & -1-4 e^{2}-6 e^{4}-4 e^{6}-e^{8}+r^{2}+e^{2} r^{2}+s^{2}+e^{2} s^{2} \\
& -6 e r u-6 e^{3} r u+2 u^{2}+13 e^{2} u^{2}+11 e^{4} u^{2}-u^{4} \\
& \quad-6 e s v-6 e^{3} s v+2 v^{2}+13 e^{2} v^{2}+11 e^{4} v^{2}-2 u^{2} v 2-v^{4}=0 .
\end{aligned}
$$

Close inspection of (3.20) reveals that

$$
F(r, s)=a_{r}\left(r+\frac{b_{r}}{2 a_{r}}\right)^{2}+a_{s}\left(s+\frac{b_{s}}{2 a_{s}}\right)^{2}-\left(\left(1+e^{2}\right)^{2}-u^{2}-v^{2}\right)^{2},
$$

where $a_{r} \equiv a_{s}:=1+e^{2}, b_{r}:=-6 e u-6 e^{3} u$ and $b_{s}:=-6 e v-6 e^{3} v$. Moreover, we observe that $\operatorname{det} M(2)_{\{1,2,3,4,6\}}=\left(1+e^{2}\right)^{2}-u^{2}-v^{2}$. It then follows that $F(r, s)=0$ precisely when $(r, s)$ is in the circle centered at $\left(-\frac{b_{r}}{2 a_{r}},-\frac{b_{s}}{2 a_{s}}\right)$ and of radius $\frac{\operatorname{det} M(2)_{\{1,2,3,4,6\}}}{\sqrt{a_{r}}}$. This completes the proof of Proposition 3.9.

Remark 3.10. Notice that the proof of Proposition 3.9 shows that in case $\operatorname{det} M(2)_{\{1,2,3,4,6\}}=0$ (i.e., $\operatorname{rank} M(2)=4$ ), the above circle reduces to the point $\left(-\frac{b_{r}}{2 a_{r}},-\frac{b_{s}}{2 a_{s}}\right)$, and $\gamma_{05}$ is then uniquely determined, as we already observed in Theorem 3.7. 


\section{CHAPTER 4}

\section{The Algebraic Variety of $\gamma$}

In Examples 2.2 and 2.5 we used the criteria of recursive consistency and normal consistency to prove that certain positive, recursively generated moment matrices $M(n)(\gamma)$ do not admit positive extensions $M(n+1)$. In view of Theorem 1.5, this implies that $\gamma$ has no finitely atomic representing measure. Does $\gamma$ have a representing measure with infinite support? In Examples 4.6 and 4.7 below, we use the variety of $\gamma$ to show that no such representing measure exists. In Example 2.14 we used the reduced $C$-block test to prove the nonexistence of flat extensions for that moment problem, but we left open the possibility that $\gamma$ has some representing measure $\mu$ with card $\operatorname{supp} \mu>\operatorname{rank} M(n)(\gamma)$. In Example 4.4 we use the variety of $\gamma$ to show that, in fact, no such measure exists. By contrast, in Examples 4.8-4.10 we employ variety techniques to construct certain minimal representing measures; of these examples, the last two have added interest because they fall outside the scope of Theorem 2.7.

For an arbitrary truncated moment problem with moment matrix $M(n)(\gamma)$, recall that the variety of $\gamma$ is given by

$$
\mathcal{V}(\gamma):=\bigcap_{\substack{p \in \mathcal{P}_{n} \\ p(Z, \bar{Z})=\mathbf{0}}} \mathcal{Z}(p) .
$$

$\mathcal{V}(\gamma)$ is a closed (possibly empty) subset of $\mathbb{C}$, satisfying $\operatorname{supp} \mu \subseteq \mathcal{V}(\gamma)$ and $\operatorname{rank} M(n) \leq \operatorname{card} \operatorname{supp} \mu \leq \operatorname{card} \mathcal{V}(\gamma)$ for every representing measure $\mu$ ((1.6) and $(1.7)$ ); we also let $\rho(\gamma):=\operatorname{card} \mathcal{V}(\gamma)-\operatorname{rank} M(n)(\gamma)$. (We mention in passing that the variety concept was implicitly used in $[\mathbf{C u F 4}$, Example 3.2] and [CuF5, Example 3.3] to determine the support of potential representing measures.)

We begin by recording several general facts concerning the size and location of the support of a representing measure.

Proposition 4.1. (cf. [CuF4, Proposition 3.1]) Let $\mu, \nu$ be representing measures for $\gamma$, and assume that $\operatorname{supp} \mu$ is contained in some algebraic curve $\mathcal{Z}(p)$ with $p \in \mathcal{P}_{n}$. Then $\operatorname{supp} \nu \subseteq \mathcal{Z}(p)$.

Proposition 4.2. An algebraic curve $\mathcal{Z}(p)$ contains no set of positive planar measure.

Proof. This is a straightforward application of Fubini's Theorem.

COROLlaRY 4.3. Let $\mu$ be a representing measure for $\gamma$ whose support contains a set of positive planar measure, and let $\nu$ be another representing measure for $\gamma$. Then $\operatorname{supp} \nu$ is not contained in any algebraic curve of degree at most $n$; moreover, $\mathcal{V}(\gamma)=\varnothing$ and $M(n)(\gamma)$ is invertible. 
In Example 2.14 we studied a moment matrix $M(3)$ with $Z^{3}=\alpha \bar{Z}^{2}$, and showed that there is no flat extension $M(4)$. We next prove that there is actually no representing measure, by showing that $\rho(\gamma)<0$.

EXAmple 4.4. (Example 2.14 revisited) The relation $z^{3}=\alpha \bar{z}^{2}, \alpha \in \mathbb{R}$, has at most two roots. For, if we write $z \equiv x+i y$, a straightforward calculation shows that the given relation is equivalent to the two (real) relations

$$
\left\{\begin{aligned}
x^{3}-3 x y^{2}-\alpha\left(x^{2}-y^{2}\right) & =0 \\
y\left(2 \alpha x-3 x^{2}+y^{2}\right) & =0 .
\end{aligned}\right.
$$

From the second equation we see that two cases arise: $y=0$ (which leads to $x=0$ and $x=\alpha$ ) and $y \neq 0$. In the latter case, we obtain $y^{2}=3 x^{2}-2 \alpha x$ from the second equation, and when we substitute this into the first equation, we get $x=0$, $x=\frac{\alpha}{2}$. If $x=0$ we get $y=0$ (a contradiction), and if $x=\frac{\alpha}{2}$ we obtain $y^{2}=-\frac{\alpha^{2}}{4}$ (again a contradiction). As a result, the only roots of $(4.1)$ are $(0,0)$ and $(\alpha, 0)$. Since $\operatorname{rank} M(3)=8$, we have $\rho(\gamma)=-6$, so there is no representing measure.

The variety test given by Proposition 4.1 can also be used as a detector of column relations of lower degree, when one relation is known, as the following example illustrates.

EXAMPle 4.5. Let $M(n)$ be a moment matrix in which $\bar{Z} Z=1+Z^{2}$. If $M(n)$ admits a representing measure, then $\operatorname{det} M(1)=0$. For, the associated real algebraic curves are $2 y^{2}=1$ and $x y=0$, from which one gets at once $\mathcal{V}(\gamma) \subseteq$ $\left\{\left(0,-\frac{\sqrt{2}}{2}\right),\left(0, \frac{\sqrt{2}}{2}\right)\right\}$. Thus, $\operatorname{rank} M(n) \leq 2$, which forces $\operatorname{det} M(1)=0$. For $n=2$, with $M(2)$ as in (3.2), the relation $\bar{Z} Z=1+Z^{2}$ and the moment matrix structure actually determine all the moments in terms of $a \equiv \gamma_{00}$. For, in $M(2), \bar{Z} Z=1+Z^{2}$ implies $d=a+c, e=b+e, e=b+f, h=c+g, g=d+h, h=c+(u+i v)$, from which we get at once $b=0, f=e, u+i v=g$ and $c+d=0$ (which further implies $a+2 c=0)$. Then $M(1)=\left(\begin{array}{ccc}a & 0 & 0 \\ 0 & \frac{a}{2} & -\frac{a}{2} \\ 0 & -\frac{a}{2} & \frac{a}{2}\end{array}\right)$, which exhibits the relation $\bar{Z}=-Z$. That in turn forces $e=0, g=\frac{a}{4}, h=-\frac{a}{4}$ and $u+i v=\frac{a}{4}$.

In Example 2.2 we exhibited a positive recursively generated $M(4)(\gamma)$, with $\bar{Z} Z^{3}$ and $\bar{Z}^{2} Z^{2}$ recursively determined, having no positive extension $M(5)$. We now show that $\mathcal{V}(\gamma)=\varnothing$, so $\gamma$ has no representing measure.

ExAmple 4.6. In Example 2.2, columns $\bar{Z} Z^{3}, \bar{Z}^{2} Z^{2}, \bar{Z}^{3} Z$ and $\bar{Z}^{4}$ are linear combinations of the preceding eleven columns, giving rise to the following three relations:

$$
\begin{aligned}
\bar{z} z^{3} & =\frac{5}{2} z^{2}+\frac{1}{\sqrt{10}} \bar{z}^{3}, \\
\bar{z}^{2} z^{2} & =-1+3 \bar{z} z
\end{aligned}
$$

and

$$
\bar{z}^{4}=-\sqrt{10} z+\sqrt{10} \bar{z}+\sqrt{\frac{5}{2}} \bar{z} z^{2}-\sqrt{\frac{5}{2}} \bar{z}^{2} z+z^{4} .
$$

We now switch to real and imaginary parts. The imaginary part of (4.2) is

$$
y\left(-25 x+3 \sqrt{\frac{5}{2}} x^{2}+10 x^{3}-\sqrt{\frac{5}{2}} y^{2}+10 x y^{2}\right)=0,
$$


which gives rise to two main cases: $y=0$ and $y \neq 0$. In the former case, the real part of $(4.2)$ becomes $x^{2}\left(\frac{-25-\sqrt{10} x+10 x^{2}}{10}\right)=0$, so two subcases arise: if $x=0$, then the real part of (4.3) becomes $0=-1$, a contradiction. We must, therefore, have $x \neq 0$. Looking at the real parts of (4.2) and (4.3) we then obtain $-25-\sqrt{10} x+10 x^{2}=0$ and $\left(x^{2}+x-1\right)\left(x^{2}-x-1\right)=0$, which admit no common solutions. Thus, we are led to examine the case $y \neq 0$. Looking now at the imaginary parts of (4.2) and (4.4), it follows that $y^{2}=\frac{50 x-3 \sqrt{10} x^{2}-20 x^{3}}{20 x-\sqrt{10}}$ and $y^{2}=\frac{\sqrt{10} x^{2}+8 x^{3}-2 \sqrt{10}}{8 x-\sqrt{10}}$, respectively. Placing these values into the expression for the real part of (4.2), we obtain two equations, which must simultaneously hold:

$$
\frac{x\left(-125 \sqrt{10}-100 x+608 \sqrt{10} x^{2}-160 x^{3}-320 \sqrt{10} x^{4}\right)}{(20 x-\sqrt{10})^{2}}=0
$$

and

$$
\frac{2\left(25-85 \sqrt{10} x-145 x^{2}+170 \sqrt{10} x^{3}+40 x^{4}-48 \sqrt{10} x^{5}\right)}{5(8 x-\sqrt{10})^{2}}=0 .
$$

Direct calculation of the roots of these equations reveals that (4.5) and (4.6) each have five real roots, but the two sets of roots are disjoint. We have thus established that $\mathcal{V}(\gamma)=\varnothing$. Thus, $M(4)$ admits no representing measure.

We next return to the special $M(4)$ problem considered in Example 2.5.

EXAMPLE 4.7. We have seen in Example 2.5 that on occasion, admissible columns, even when they arise from conjugate relations, need not be normal with respect to one another. That the relation $\bar{Z} Z^{3}=\frac{5}{2} Z^{2}+\frac{3}{5} \bar{Z}^{3}$ gives rise to admissible columns forming a non-normal band is sufficient to show that $M(4)$ admits no positive extension, and that therefore no 13-atomic representing measure exists (Theorem 1.1). More generally, the lack of normal consistency implies (via Theorem 1.5) the non-existence of finitely atomic representing measures. One might wonder about the existence of non-atomic representing measures. We shall now use the variety test in Proposition 4.1 to see that the moment matrix in Example 2.5 admits no representing measure whatsoever. We shall do this by explicitly showing that $\mathcal{V}(\gamma)$ has exactly 11 points, so $\rho(\gamma)<0$. As usual, let $z \equiv x+i y$. The relation $\bar{z} z^{3}=\frac{5}{2} z^{2}+\frac{3}{5} \bar{z}^{3}$ implies that

$$
-25 x^{2}-6 x^{3}+10 x^{4}+25 y^{2}+18 x y^{2}-10 y^{4}=0
$$

and

$$
y\left(-25 x+9 x^{2}+10 x^{3}-3 y^{2}+10 x y^{2}\right)=0 .
$$

If $y=0,(4.7)$ gives rise to the three values $x=0, x \cong-1.30935$ and $x \cong 1.90935$. If $y \neq 0$, then (4.8) forces

$$
y^{2}=\frac{25 x-9 x^{2}-10 x^{3}}{10 x-3}
$$

When this value of $y^{2}$ is replaced into (4.7) we obtain the five values $x=0, x \cong$ $-1.54469, x \cong-0.404611, x \cong 0.590021$ and $x \cong 1.05928$. Since $y \neq 0,(4.9)$ forces $x \neq 0$, and each of the remaining four $x$-values gives rise to two $y$-values via (4.9), for a total of 11 different points in $\mathcal{V}(\gamma)$. 
In Example 2.15 we used Theorem 2.7 to reduce an $M(3)$ flat extension problem (with $\bar{Z} Z^{2}$ recursively determined) to the $C$-block test $C_{11}=C_{22}$. We now wish to study a case of this example in which we can find a flat extension of $M(3)$.

EXAmPle 4.8. For $g, k \in \mathbb{R}$, let

$$
M(3):=\left(\begin{array}{cccccccccc}
1 & 0 & 0 & 0 & 1 & 0 & 0 & 0 & 0 & 0 \\
0 & 1 & 0 & 0 & 0 & 0 & 0 & g & 0 & 0 \\
0 & 0 & 1 & 0 & 0 & 0 & 0 & 0 & g & 0 \\
0 & 0 & 0 & g & 0 & 0 & 0 & 0 & 0 & k \\
1 & 0 & 0 & 0 & g & 0 & 0 & 0 & 0 & 0 \\
0 & 0 & 0 & 0 & 0 & g & k & 0 & 0 & 0 \\
0 & 0 & 0 & 0 & 0 & k & g^{2} & 0 & 0 & 0 \\
0 & g & 0 & 0 & 0 & 0 & 0 & g^{2} & 0 & 0 \\
0 & 0 & g & 0 & 0 & 0 & 0 & 0 & g^{2} & 0 \\
0 & 0 & 0 & k & 0 & 0 & 0 & 0 & 0 & g^{2}
\end{array}\right) .
$$

One checks that $[M(3)]_{6}>0$ if and only if $g>1$, that $\operatorname{det}[M(3)]_{7}=g(g-1)\left(g^{3}-k^{2}\right)$ and that $\operatorname{det}[M(3)]_{\{1,2,3,4,5,6,7,10\}}=(g-1)\left(g^{3}-k^{2}\right)^{2}$. Thus, the conditions $g>1$, $g^{3}>k^{2}$ are sufficient to guarantee that $\operatorname{rank} M(3) \geq 8$. Moreover, a straightforward calculation shows that

$$
\bar{Z} Z^{2}=g Z
$$

and

$$
\bar{Z}^{2} Z=g \bar{Z},
$$

so rank $M(3)=8$ when $g^{3}>k^{2}$. (From (4.10) we see that $\mathcal{V}\left(\gamma^{(6)}\right)$ is determined by the single relation $z\left(|z|^{2}-g\right)=0$, whence card $\mathcal{V}\left(\gamma^{(6)}\right)=\infty$.) It follows readily from (4.10) and (4.11) that $\bar{Z} Z^{3}:=g Z^{2}, \bar{Z}^{2} Z^{2}:=g \bar{Z} Z$, and $\bar{Z}^{3} Z:=g \bar{Z}^{2}$ are admissible for $M(3)$, and Proposition 2.3 implies that they form a normal band. Since (4.11) is obtained from (4.10) by conjugation, we can restrict attention to (4.10). In a flat extension $M(4)$ of $M(3)$, observe that (4.10) immediately implies

$$
\left[\bar{Z} Z^{3}\right]_{10}=g\left[Z^{2}\right]_{10}=\left(0,0,0, g^{2}, 0,0,0,0,0, g k\right)^{T},
$$

which forces $\left[Z^{4}\right]_{10}=(0,0, k, 0,0,0,0,0, g k, w)^{T}$ by normality, where $w$ is a free parameter. But $\left[Z^{4}\right]_{10}=k[\bar{Z}]_{10}-\frac{k w}{g^{3}-k^{2}}\left[Z^{2}\right]_{10}+\frac{g w}{g^{3}-k^{2}}\left[\bar{Z}^{3}\right]_{10}$, so by the Extension Principle, the same linear combination must hold in $\mathcal{C}_{M(4)}$, that is

$$
Z^{4}=k \bar{Z}-\frac{k w}{g^{3}-k^{2}} Z^{2}+\frac{g w}{g^{3}-k^{2}} \bar{Z}^{3} .
$$

If we define $\bar{Z}^{4}$ via symmetry, then it follows that the resulting block $B$ is a moment matrix extension block satisfying $\operatorname{Ran} B \subseteq \operatorname{Ran} M(3)$. According to Example 2.15, the necessary and sufficient condition for the existence of a flat extension $M(4)$ is equality of the $(1,1)$ and $(2,2)$ entries of the $C$ block of $[M(3) ; B]$, that is,

$$
k \gamma_{50}-\frac{k w}{g^{3}-k^{2}} \gamma_{42}+\frac{g \bar{w}}{g^{3}-k^{2}} \gamma_{70}=g \gamma_{33} .
$$

Equivalently, $k^{2}+\frac{g}{g^{3}-k^{2}}|w|^{2}=g^{3}$, so that $|w|^{2}=\frac{\left(g^{3}-k^{2}\right)^{2}}{g}$, which means that $w$ can be chosen anywhere on the circle centered at the origin with radius $\frac{g^{3}-k^{2}}{\sqrt{g}}$. (We note in passing that each value of $w$ gives rise to a distinct representing measure, so $\gamma$ 
admits infinitely many 8 -atomic representing measures.) We conclude this example by showing how variety techniques can be used to explicitly compute a representing measure in a special case.

To simplify the remaining calculations, we let $g:=u^{2}, k:=\frac{u^{3}}{2}$, and $w:=\frac{3}{4} u^{5}$. Let $M[w]$ be the associated flat extension. With $z \equiv x+i y,(4.10)$ and (4.13) imply that the variety associated with $M[w]$ is obtained as the intersection of the following four algebraic curves:

$$
\begin{aligned}
x\left(x^{2}+y^{2}-u^{2}\right) & =0, \\
y\left(x^{2}+y^{2}-u^{2}\right) & =0, \\
x^{4}+y^{4}-6 x^{2} y^{2}-\frac{u^{3}}{2} x+\frac{u^{2}}{2}\left(x^{2}-y^{2}\right)-u\left(x^{3}-3 x y^{2}\right) & =0,
\end{aligned}
$$

and

$$
4 x^{3} y-4 x y^{3}+\frac{u^{3}}{2} y+u^{2} x y+3 u x^{2} y-u y^{3}=0 .
$$

One checks that only the following eight points satisfy (4.15), (4.16), (4.17) and (4.18) simultaneously:

$$
\begin{array}{ll}
\left(x_{0}, y_{0}\right)=(0,0), & \left(x_{1}, y_{1}\right)=(u, 0) \\
\left(x_{2}, y_{2}\right)=\left(-\frac{u}{2}-\frac{\sqrt{2}}{4} u, \frac{\sqrt{5-2 \sqrt{2}}}{2 \sqrt{2}} u\right), & \left(x_{3}, y_{3}\right)=\left(-\frac{u}{2}-\frac{\sqrt{2}}{4} u,-\frac{\sqrt{5-2 \sqrt{2}}}{2 \sqrt{2}} u\right), \\
\left(x_{4}, y_{4}\right)=\left(-\frac{u}{2}+\frac{\sqrt{2}}{4} u, \frac{\sqrt{5+2 \sqrt{2}}}{2 \sqrt{2}} u\right), & \left(x_{5}, y_{5}\right)=\left(-\frac{u}{2}+\frac{\sqrt{2}}{4} u,-\frac{\sqrt{5+2 \sqrt{2}}}{2 \sqrt{2}} u\right), \\
\left(x_{6}, y_{6}\right)=\left(\frac{u}{2}, \frac{\sqrt{3}}{2} u\right) & \left(x_{7}, y_{7}\right)=\left(\frac{u}{2},-\frac{\sqrt{3}}{2} u\right) .
\end{array}
$$

To calculate the densities associated with the atoms whose real and imaginary parts are given by these eight points, we consider the corresponding Vandermonde matrix $V(u)$, built using the complex numbers $z_{k}:=x_{k}+i y_{k}(0 \leq k \leq 7)$. A calculation reveals that with

$$
\rho:=\left(1-\frac{1}{u^{2}}, \frac{3}{17 u^{2}}, \frac{16+3 \sqrt{2}}{119 u^{2}}, \frac{16+3 \sqrt{2}}{119 u^{2}}, \frac{16-3 \sqrt{2}}{119 u^{2}}, \frac{16-3 \sqrt{2}}{119 u^{2}}, \frac{1}{7 u^{2}}, \frac{1}{7 u^{2}}\right),
$$

we have

$$
V(u) \boldsymbol{\rho}^{T}=\left(1,0,0,0,0, \frac{1}{2} u^{3}, 0, \frac{3}{4} u^{5}\right)=\left(\gamma_{00}, \gamma_{01}, \gamma_{02}, \gamma_{03}, \gamma_{04}, \gamma_{05}, \gamma_{06}, \gamma_{07}\right)^{T},
$$

so $\mu:=\sum_{k=0}^{7} \rho_{k} z_{k}$ is the desired representing measure.

We next turn to moment problems that fall outside the scope of Theorem 2.7. We first consider a flat extension problem for $M(3)$ in which $\left\{1, Z, \bar{Z}, Z^{2}, \bar{Z} Z, \bar{Z}^{2}, Z^{3}\right\}$ is a basis for $\mathcal{C}_{M(3)}, \bar{Z} Z^{2}$ and $\bar{Z}^{2} Z$ are recursively determined, but $\bar{Z}^{3}=Z^{3}$ (so that Theorem 2.7 does not apply). 
EXAMPLE 4.9. Let

$$
M \equiv M(3):=\left(\begin{array}{cccccccccc}
1 & 0 & 0 & 0 & 1 & 0 & 0 & 0 & 0 & 0 \\
0 & 1 & 0 & 0 & 0 & 0 & 0 & \alpha & 0 & 0 \\
0 & 0 & 1 & 0 & 0 & 0 & 0 & 0 & \alpha & 0 \\
0 & 0 & 0 & \alpha & 0 & 0 & 0 & 0 & u & 0 \\
1 & 0 & 0 & 0 & \alpha & 0 & u & 0 & 0 & u \\
0 & 0 & 0 & 0 & 0 & \alpha & 0 & u & 0 & 0 \\
0 & 0 & 0 & 0 & u & 0 & \beta & 0 & 0 & \delta \\
0 & \alpha & 0 & 0 & 0 & u & 0 & \beta & 0 & 0 \\
0 & 0 & \alpha & u & 0 & 0 & 0 & 0 & \beta & 0 \\
0 & 0 & 0 & 0 & u & 0 & \delta & 0 & 0 & \beta
\end{array}\right),
$$

with $\alpha, \beta, \delta$ and $u$ real numbers. We observe at once that with $\alpha>1$ we guarantee $\operatorname{det}[M]_{6}>0$, and that the additional condition $\beta>\frac{u^{2}}{\alpha-1}$ implies $[M]_{7}$ positive and invertible. We next impose the column relation

$$
\bar{Z} Z^{2}=\alpha Z+\frac{u}{\alpha} \bar{Z}^{2}
$$

which implies $[M]_{8} \geq 0$. Thus,

$$
\alpha^{2}+\frac{u^{2}}{\alpha}=\beta \quad\left(>\frac{u^{2}}{\alpha-1}\right),
$$

whence

$$
0<u<\sqrt{\alpha^{3}(\alpha-1)}
$$

and

$$
\alpha^{2}<\beta<\alpha^{3}
$$

We also have

$$
\bar{Z}^{2} Z=\alpha \bar{Z}+\frac{u}{\alpha} Z^{2}
$$

which, together with the positivity of $[M]_{8}$ and Smul'jan's Criterion $[\mathbf{C u F 1}$, Proposition 2.3], implies $[M]_{9} \geq 0$. Let $p(z, \bar{z}):=\alpha z+\frac{u}{\alpha} \bar{z}^{2}$. Recursive consistency for $M$ is equivalent to $(\bar{z} p)(Z, \bar{Z})=(z \bar{p})(Z, \bar{Z})$, which reduces to the condition $\beta=\delta$. With this condition, we automatically have $\bar{Z}^{3}=Z^{3}$, so $M \geq 0$ (by the positivity of $[M]_{9}$ and Smul'jan's Criterion once again).

Thus, with $\beta=\delta$, we have $M$ positive, recursively generated, and recursively consistent. In constructing a flat moment matrix extension block $B$, the admissible columns are

$$
\begin{aligned}
\bar{Z} Z^{3} & =\alpha Z^{2}+\frac{u}{\alpha} \bar{Z}^{2} Z \\
\bar{Z}^{2} Z^{2} & =\alpha \bar{Z} Z+\frac{u}{\alpha} \bar{Z}^{3}
\end{aligned}
$$

and

$$
\bar{Z}^{3} Z=\alpha \bar{Z}^{2}+\frac{u}{\alpha} \bar{Z} Z^{2}
$$


These columns form a normal band, so $M$ is normally consistent. Calculations using (4.24), (4.25) and (4.26) show that the block $B_{34}$ is of the form

$$
\left(\begin{array}{ccccc}
s & 0 & \alpha u+\frac{\beta u}{\alpha} & 0 & \bar{v} \\
x & 0 & 0 & \alpha u+\frac{\beta u}{\alpha} & \bar{y} \\
y & \alpha u+\frac{\beta u}{\alpha} & 0 & 0 & \bar{x} \\
v & 0 & \alpha u+\frac{\beta u}{\alpha} & 0 & \bar{s}
\end{array}\right) .
$$

Now $Z^{4}$ in block $B$ must be of the form $\mathbf{r} \equiv(0, u, 0,0,0, \beta, s, x, y, v)^{T}$. Let $I$ denote the basis for $\mathcal{C}_{M}$ given by $\left\{1, Z, \bar{Z}, Z^{2}, \bar{Z} Z, \bar{Z}^{2}, Z^{3}\right\}$, and let $[\mathbf{r}]_{I}:=(0, u, 0,0,0, \beta, s)^{T}$. To ensure normality in $B$, we require $s=0$, whence $[\mathbf{r}]_{I}=u[Z]_{I}+\frac{\beta}{\alpha}\left[\bar{Z}^{2}\right]_{I}$; thus, we are forced to define

$$
Z^{4}:=u Z+\frac{\beta}{\alpha} \bar{Z}^{2}
$$

Now

$$
\begin{aligned}
& x \equiv\left\langle z^{4}, \bar{z} z^{2}\right\rangle_{B}=u \gamma_{22}+\frac{\beta}{\alpha} \gamma_{41}=\alpha u+\frac{\beta}{\alpha} u=\left\langle\bar{z} z^{3}, \bar{z}^{2} z\right\rangle_{B}, \\
& y \equiv\left\langle z^{4}, \bar{z}^{2} z\right\rangle_{B}=u \gamma_{13}+\frac{\beta}{\alpha} \gamma_{32}=0=\left\langle\bar{z} z^{3}, \bar{z}^{3}\right\rangle_{B},
\end{aligned}
$$

and

$$
v \equiv\left\langle z^{4}, \bar{z}^{3}\right\rangle_{B}=u \gamma_{04}+\frac{\beta}{\alpha} \gamma_{23}=0 .
$$

Thus, there exists a unique moment matrix extension block $B$ satisfying Ran $B \subseteq$ Ran $M$. Since the admissible columns are $\bar{Z} Z^{3}, \bar{Z}^{2} Z^{2}$ and $\bar{Z}^{3} Z$, Proposition 2.6 implies that the $C$ block of $[M ; B]$ is Toeplitz if and only if $C_{11}=C_{22}$. This is equivalent to

$$
u \gamma_{41}+\frac{\beta}{\alpha} \gamma_{60}=\alpha \gamma_{33}+\frac{u}{\alpha} \gamma_{52}
$$

or

$$
u^{2}+\frac{\beta^{2}}{\alpha}=\alpha \beta+\frac{u}{\alpha}\left(\alpha u+\frac{\beta u}{\alpha}\right),
$$

and the latter identity follows from (4.20). Thus $M$ has a unique flat moment matrix extension.

A calculation shows that $\bar{z} z^{2}=\alpha z+\frac{u}{\alpha} \bar{z}^{2}$ has exactly $7(=\operatorname{rank} M)$ solutions, which thus provide the support of the unique representing measure; these solutions are as follows:

$$
\begin{array}{ll}
z_{0}=0 ; \\
z_{1}=\frac{u+\sqrt{u^{2}+4 \alpha^{3}} ;}{2 \alpha} ; & z_{2}=\frac{u-\sqrt{u^{2}+4 \alpha^{3}}}{2 \alpha} ; \\
z_{3}=-\frac{z_{2}}{2}+i \sqrt{\frac{\alpha^{2}-2 u z_{2}-\alpha z_{2}^{2}}{\alpha}} ; & z_{4}=\bar{z}_{3} ; \\
z_{5}=-\frac{z_{1}}{2}+i \sqrt{\frac{\alpha^{2}-2 u z_{1}-\alpha z_{1}^{2}}{\alpha}} ; & z_{6}=\bar{z}_{5} .
\end{array}
$$


Since $M(4)$ is a flat extension of $M(3)$, we may now find the corresponding densities via (1.3). These densities are

$$
\begin{gathered}
\rho_{0}=1-\frac{\beta}{\alpha^{3}} ; \\
\rho_{1}=\rho_{5}=\rho_{6}=\frac{\beta}{6 \alpha^{3}}-\frac{u\left(\beta+2 \alpha^{2}\right)}{6 \alpha^{3} \sqrt{u^{2}+4 \alpha^{3}}} ; \\
\rho_{2}=\rho_{3}=\rho_{4}=\frac{\beta}{6 \alpha^{3}}-\frac{u\left(\beta+2 \alpha^{2}\right)}{6 \alpha^{3} \sqrt{u^{2}+4 \alpha^{3}}}
\end{gathered}
$$

(observe that by $(4.22), \frac{\beta}{\alpha^{3}}<1$, so $0<\rho_{0}<1$; similarly, an easy calculation shows that $\rho_{1}$ and $\rho_{2}$ are positive, in keeping with Theorem 1.4).

For our last example we consider an $M(4)$ flat extension problem in which the first 12 columns form a basis for $\mathcal{C}_{M(4)}, \bar{Z}^{2} Z^{2}$ is recursively determined, but $\bar{Z}^{3} Z$ and $\bar{Z}^{4}$ are not recursively determined, so Theorem 2.7 does not apply.

EXAMPLE 4.10. We consider the moment problem for

$$
M(4) \equiv\left(\begin{array}{ccccccccccccccc}
1 & 0 & 0 & 0 & 1 & 0 & 0 & 0 & 0 & 0 & 0 & 0 & \alpha & 0 & 0 \\
0 & 1 & 0 & 0 & 0 & 0 & 0 & \alpha & 0 & 0 & 0 & 0 & 0 & 0 & 0 \\
0 & 0 & 1 & 0 & 0 & 0 & 0 & 0 & \alpha & 0 & 0 & 0 & 0 & 0 & 0 \\
0 & 0 & 0 & \alpha & 0 & 0 & 0 & 0 & 0 & 0 & 0 & \beta & 0 & 0 & 0 \\
1 & 0 & 0 & 0 & \alpha & 0 & 0 & 0 & 0 & 0 & 0 & 0 & \beta & 0 & 0 \\
0 & 0 & 0 & 0 & 0 & \alpha & 0 & 0 & 0 & 0 & 0 & 0 & 0 & \beta & 0 \\
0 & 0 & 0 & 0 & 0 & 0 & \beta & 0 & 0 & 0 & 0 & 0 & 0 & 0 & 0 \\
0 & \alpha & 0 & 0 & 0 & 0 & 0 & \beta & 0 & 0 & 0 & 0 & 0 & 0 & 0 \\
0 & 0 & \alpha & 0 & 0 & 0 & 0 & 0 & \beta & 0 & 0 & 0 & 0 & 0 & 0 \\
0 & 0 & 0 & 0 & 0 & 0 & 0 & 0 & 0 & \beta & 0 & 0 & 0 & 0 & 0 \\
0 & 0 & 0 & 0 & 0 & 0 & 0 & 0 & 0 & 0 & \delta & 0 & 0 & u & 0 \\
0 & 0 & 0 & \beta & 0 & 0 & 0 & 0 & 0 & 0 & 0 & \delta & 0 & 0 & u \\
\alpha & 0 & 0 & 0 & \beta & 0 & 0 & 0 & 0 & 0 & 0 & 0 & \delta & 0 & 0 \\
0 & 0 & 0 & 0 & 0 & \beta & 0 & 0 & 0 & 0 & u & 0 & 0 & \delta & 0 \\
0 & 0 & 0 & 0 & 0 & 0 & 0 & 0 & 0 & 0 & 0 & u & 0 & 0 & \delta
\end{array}\right)
$$

With $\alpha>1, \beta>\alpha^{2}$ and $\delta>\frac{\beta^{2}}{\alpha}$, the Nested Determinants Test implies that $M(4)_{12}$ is positive and invertible. With $\delta=\frac{\alpha^{3}-2 \alpha \beta+\beta^{2}}{\alpha-1}\left(>\frac{\beta^{2}}{\alpha}\right)$, we have

$$
\bar{Z}^{2} Z^{2}=\frac{\alpha^{2}-\beta}{\alpha-1} 1+\frac{\beta-\alpha}{\alpha-1} \bar{Z} Z ;
$$

moreover, $\operatorname{det} M(4)_{13}=0$, so $M(4)_{13} \geq 0$. With $u=\sqrt{\delta\left(\delta-\frac{\beta^{2}}{\alpha}\right)}$ we have

$$
\bar{Z}^{3} Z=\frac{\beta}{\alpha} \bar{Z}^{2}+\frac{u}{\delta} Z^{4}
$$

and

$$
\bar{Z}^{4}=-\frac{\beta \delta}{\alpha u} Z^{2}+\frac{\delta}{u} \bar{Z} Z^{3} ;
$$

since $\operatorname{rank} M(4)=\operatorname{rank} M(4)_{13}=12$, it follows that $M(4) \geq 0$ (by Proposition 2.1).

We next proceed to define a moment matrix extension block $B$ satisfying $\operatorname{Ran} B \subseteq \operatorname{Ran} M(4)$. To construct a flat extension of $M(4)$, notice that in view 
of (4.28) and (4.29), any recursively generated moment matrix extension of $M(4)$ must satisfy

$$
\bar{Z}^{4} Z=\frac{\beta}{\alpha} \bar{Z}^{3}+\frac{u}{\delta} \bar{Z} Z^{4}
$$

and

$$
\bar{Z}^{4} Z=-\frac{\beta \delta}{\alpha u} Z^{3}+\frac{\delta}{u} \bar{Z} Z^{4},
$$

respectively. From (4.30) and (4.31) we see that

$$
\bar{Z} Z^{4}=\frac{\delta}{\beta} Z^{3}+\frac{u}{\beta} \bar{Z}^{3} .
$$

Our strategy is then to define $\bar{Z} Z^{4}$ in block $B$ by (4.32). At the same time, (4.27) allows us to define $\bar{Z}^{2} Z^{3}$ by the formula

$$
\bar{Z}^{2} Z^{3}:=\frac{\alpha^{2}-\beta}{\alpha-1} Z+\frac{\beta-\alpha}{\alpha-1} \bar{Z} Z^{2}
$$

and a straightforward calculation shows that $\bar{Z} Z^{4}$ and $\bar{Z}^{2} Z^{3}$ form a normal band in $B$. Using normality, we can generate $\left[Z^{5}\right]_{I}$ from $\bar{Z} Z^{4}$, where $I$ denotes the basis for $\mathcal{C}_{M(4)}$ consisting of the first 12 columns. A calculation reveals, however, that

$$
\left[Z^{5}\right]_{I}=-\frac{\alpha u}{\beta-\alpha^{2}}[\bar{Z}]_{I}+\frac{u}{\beta-\alpha^{2}}\left[\bar{Z}^{2} Z\right]_{I}
$$

Of course, this leads us to define

$$
Z^{5}:=-\frac{\alpha u}{\beta-\alpha^{2}} \bar{Z}+\frac{u}{\beta-\alpha^{2}} \bar{Z}^{2} Z
$$

and another calculation shows that $Z^{5}$ is then normal with respect to $\bar{Z} Z^{4}$ in $B$. The remaining columns of block $B$ are defined by symmetry (cf. Proposition 2.3 and $[\mathbf{C u F 4}$, Lemma 3.10]); the resulting moment matrix extension block $B$ clearly satisfies $\operatorname{Ran} B \subseteq \operatorname{Ran} M(4)$, and is the unique such block. (Incidentally, observe that $B_{54}=0$.)

We next consider the $C$ block of $[M(4) ; B]$; since $\bar{Z}^{2} Z^{3}$ and $\bar{Z}^{3} Z^{2}$ form a normal band of admissible columns in $B$, the reduced $C$-block test implies that $C$ is Toeplitz 
if and only if $C_{11}=C_{22}=C_{33}, C_{21}=C_{32}$, and $C_{41}=C_{52}$. Now,

$$
\begin{aligned}
C_{11}=C_{22} \Longleftrightarrow & \left\langle z^{5}, z^{5}\right\rangle_{C}=\left\langle\bar{z} z^{4}, \bar{z} z^{4}\right\rangle_{C} \\
\Longleftrightarrow & \left\langle-\frac{\alpha u}{\beta-\alpha^{2}} \bar{z}+\frac{u}{\beta-\alpha^{2}} \bar{z}^{2} z, z^{5}\right\rangle_{B^{*}}=\left\langle\frac{\delta}{\beta} z^{3}+\frac{u}{\beta} \bar{z}^{3}, \bar{z} z^{4}\right\rangle_{B^{*}} \\
& \quad(\text { using }(4.34) \text { and }(4.32), \text { respectively) } \\
\Longleftrightarrow & \frac{u^{2}}{\beta-\alpha^{2}}=\frac{\delta^{2}+u^{2}}{\beta} \\
\Longleftrightarrow & \delta=\frac{\alpha \beta^{2}}{2 \alpha^{2}-\beta} \\
& \left(\text { since } \delta>0, \text { this entails } 2 \alpha^{2}>\beta\right) \\
& \Longleftrightarrow \frac{\alpha^{3}-2 \alpha \beta+\beta^{2}}{\alpha-1}=\frac{\alpha \beta^{2}}{2 \alpha^{2}-\beta} \\
& \left(\beta^{2}-3 \alpha \beta+2 \alpha^{3}\right)\left(\beta-\alpha^{2}\right)=0 \\
& \Longleftrightarrow \beta=\frac{\alpha(3 \pm \sqrt{9-8 \alpha})}{2} .
\end{aligned}
$$

These values require $1<\alpha<\frac{9}{8}$ and do satisfy $\alpha^{2}<\beta<2 \alpha^{2}$. Next, from (4.33) and (4.32) we have

$$
C_{22}=C_{33} \Longleftrightarrow \frac{\alpha^{2}-\beta+(\beta-\alpha) \delta}{\alpha-1}=\frac{\delta^{2}+u^{2}}{\beta},
$$

and this condition is equivalent to (4.35). Similarly, from (4.32) and (4.34) we see that

$$
C_{41}=C_{52} \Longleftrightarrow \frac{u(-\alpha \beta+\delta)}{\beta-\alpha^{2}}=\frac{2 u \delta}{\beta} ;
$$

this reduces to $\delta=\frac{\alpha \beta^{2}}{2 \alpha^{2}-\beta}$, which is equivalent to (4.35). We also have $C_{21}=C_{32}=$ 0 . Thus, (4.35) implies that $M(4)$ has a unique flat extension.

In summary, with $\alpha>1, \beta>\alpha^{2}, \delta=\frac{\alpha^{3}-2 \alpha \beta+\beta^{2}}{\alpha-1}, u=\sqrt{\delta\left(\delta-\frac{\beta^{2}}{\alpha}\right)}, M(4)$ is positive, recursively generated, and recursively and normally consistent. There exists a unique moment matrix extension block $B$ satisfying Ran $B \subseteq \operatorname{Ran} M(4)$, but $M(4)$ has a flat extension (necessarily unique, namely $[M(4) ; B]$ ) if and only if $1<\alpha<\frac{9}{8}$ and $\beta=\frac{\alpha(3 \pm \sqrt{9-8 \alpha})}{2}$.

Under the conditions just described, $\gamma$ has a unique 12-atomic representing measure. Does $\gamma$ admit other representing measures? To resolve this question, we next compute $\mathcal{V}(\gamma)$. In $\mathcal{C}_{M(4)}$ we have the following relations:

$$
\begin{aligned}
\bar{Z}^{2} Z^{2} & =-\frac{\beta-\alpha^{2}}{\alpha-1} 1+\frac{\beta-\alpha}{\alpha-1} \bar{Z} Z, \\
\bar{Z}^{3} Z & =\frac{\beta}{\alpha} \bar{Z}^{2}+\frac{u}{\delta} Z^{4},
\end{aligned}
$$

and

$$
\bar{Z}^{4}=-\frac{\beta \delta}{\alpha u} Z^{2}+\frac{\delta}{u} \bar{Z} Z^{3}
$$


from symmetry, it follows that (4.37) and (4.38) are equivalent, so for the purposes of computing $\mathcal{V}(\gamma)$ we consider only (4.37). Let $\theta:=-\frac{\beta-\alpha^{2}}{\alpha-1}, \eta:=\frac{\beta-\alpha}{\alpha-1}, z \equiv x+i y$; (4.36) becomes $\left(x^{2}+y^{2}\right)^{2}=\theta+\eta\left(x^{2}+y^{2}\right)$, or

$$
x^{2}+y^{2}=\frac{\eta \pm \sqrt{4 \theta+\eta^{2}}}{2} .
$$

Note that $4 \theta+\eta^{2}>0$ for $\alpha>1$; indeed, if $p(\beta):=\beta^{2}+(4-6 \alpha) \beta+4 \alpha^{3}-3 \alpha^{2}$ $\left(\equiv 4 \theta+\eta^{2}\right)$, the discriminant of $p$ is $-16(\alpha-1)^{3}<0$, and since $p(0)=\alpha^{2}(4 \alpha-3)>0$, we see that $p>0$ everywhere. Now define $r_{1}:=\frac{\eta+\sqrt{4 \theta+\eta^{2}}}{2}$ and $r_{2}:=\frac{\eta-\sqrt{4 \theta+\eta^{2}}}{2}$. Since $\theta<0$, it follows that $0<r_{2}<r_{1}$, so $\mathcal{V}(\gamma)$ is contained in the union of the two circles centered at the origin of radii $\sqrt{r_{1}}$ and $\sqrt{r_{2}}$; for notational convenience, we shall represent the union of these two circles by the equation

$$
x^{2}+y^{2}=r
$$

Next, (4.37) is equivalent to the real system

$$
\alpha(\delta-u) x^{4}-\beta \delta x^{2}+6 \alpha u x^{2} y^{2}+\beta \delta y^{2}-\alpha(\delta+u) y^{4}=0
$$

and

$$
x y\left(\beta \delta-\alpha(\delta+2 u) x^{2}+\alpha(2 u-\delta) y^{2}\right)=0
$$

If $x^{2}+y^{2}=r$ with $x \neq 0, y \neq 0$, then $(x, y)$ satisfies (4.42) if and only if $y^{2}=$ $\frac{\alpha r(\delta+2 u)-\beta \delta}{4 \alpha u}$. It follows that for $r=r_{1}$ (and $\left.x \neq 0, y \neq 0\right)$, there are at most 4 points satisfying (4.40) and (4.42), and similarly for $r=r_{2}$. Thus $\mathcal{V}(\gamma)$ contains at most 8 points with nonzero $x$ and $y$ coordinates. When $x=0,(4.40)$ implies that $y \neq 0$, and (4.41) reduces to $y^{2}=\frac{\beta \delta}{\alpha(\delta+u)}$, contributing two more points to $\mathcal{V}(\gamma)$. Finally, if $y=0$ then (4.40) says that $x \neq 0$, and (4.41) implies that $x^{2}=\frac{\beta \delta}{\alpha(\delta-u)}$. (Incidentally, since $\frac{\beta \delta}{\alpha(\delta+u)}<\frac{\beta \delta}{\alpha(\delta-u)}$, (4.40) and the previous argument show that $r_{1}=\frac{\beta \delta}{\alpha(\delta-u)}$ and $r_{2}=\frac{\beta \delta}{\alpha(\delta+u)}$. $)$

We can thus conclude that $\operatorname{card} \mathcal{V}(\gamma) \leq 12=\operatorname{rank} M(4)$, so it follows from [CuF4] and (1.7) that if $\gamma$ admits a representing measure, then it has to be unique, with support $\mathcal{V}(\gamma)$. At the same time, such a representing measure must arise from a flat moment matrix extension $M(5)$ of $M(4)$. In other words, $\gamma$ admits a representing measure $\mu$ if and only if $[M(4) ; B]$, as constructed above, is a flat moment matrix extension, in which case $\mu$ is 12 -atomic. As we know, this happens if and only if $1<\alpha<\frac{9}{8}$ and $\beta=\frac{\alpha(3 \pm \sqrt{9-8 \alpha})}{2}$. 
We next compute the atoms in the case $\beta=\frac{\alpha(3+\sqrt{9-8 \alpha})}{2}$. The 12 atoms of the unique representing measure $\mu$ are $z_{j}:=x_{j}+i y_{j}(0 \leq j \leq 11)$, where

$$
\begin{array}{rlrl}
\left(x_{0}, y_{0}\right) & :=\left(0, \sqrt{r_{2}}\right), & \left(x_{1}, y_{1}\right):=\left(0,-\sqrt{r_{2}}\right), & \\
\left(x_{2}, y_{2}\right):=\left(\sqrt{r_{1}}, 0\right), & \left(x_{3}, y_{3}\right):=\left(-\sqrt{r_{1}}, 0\right), & \\
y_{4} \equiv y_{5}:=\sqrt{\frac{\alpha r_{1}(\delta+2 u)-\beta \delta}{4 \alpha u},} & x_{4}:=\sqrt{r_{1}-y_{4}^{2}}, & x_{5}:=-x_{4} \\
y_{6} \equiv y_{7}:=-\sqrt{\frac{\alpha r_{1}(\delta+2 u)-\beta \delta}{4 \alpha u}}, & x_{6}:=\sqrt{r_{1}-y_{6}^{2}}, & x_{7}:=-x_{6} \\
y_{8} \equiv y_{9}:=\sqrt{\frac{\alpha r_{2}(\delta+2 u)-\beta \delta}{4 \alpha u}}, & x_{8}:=\sqrt{r_{2}-y_{8}^{2}}, & x_{9}:=-x_{8} \\
y_{10} \equiv y_{11}:=-\sqrt{\frac{\alpha r_{2}(\delta+2 u)-\beta \delta}{4 \alpha u}}, & x_{10}:=\sqrt{r_{2}-y_{10}^{2}}, & x_{11}:=-x_{10} .
\end{array}
$$

Our next goal is to compute the densities $\rho_{0}, \ldots, \rho_{11}$ of $\mu$ via (1.3). To this end, we must first calculate certain high-order analytic moments of $\mu$ which are not part of the initial data $\gamma$, namely $\gamma_{0,9}[\mu], \gamma_{0,10}[\mu]$ and $\gamma_{0,11}[\mu]$. Let $s_{1}:=-\frac{\alpha u}{\beta-\alpha^{2}}$ and $s_{2}:=\frac{u}{\beta-\alpha^{2}}$; (4.34) shows that $Z^{5}=s_{1} \bar{Z}+s_{2} \bar{Z}^{2} Z$ in $M^{\prime} \equiv M(5)(\gamma[\mu]):=$ $[M(4) ; B]$, so $\gamma_{0,9}[\mu]=\left(s_{1} \bar{Z}+s_{2} \bar{Z}^{2} Z, \hat{\bar{z}}^{4}\right)=s_{1} \gamma_{14}+s_{2} \gamma_{25}=0$, and $\gamma_{0,10}[\mu]=$ $\left(s_{1} \bar{Z}+s_{2} \bar{Z}^{2} Z, \hat{\bar{z}}^{5}\right)=s_{1} \gamma_{15}+s_{2} \gamma_{26}=0$. From Theorem 1.4(ii), $M^{\prime}$ has a flat extension to $M^{\prime \prime} \equiv M(6)\{\gamma[\mu]\}$, and in $\mathcal{C}_{M^{\prime \prime}}$ we have $Z^{6}=s_{1} \bar{Z} Z+s_{2} \bar{Z}^{2} Z^{2}$; thus, $\gamma_{0,11}[\mu]=\left(s_{1} \bar{Z} Z+s_{2} \bar{Z}^{2} Z^{2}, \hat{\bar{z}}^{5}\right)=s_{1} \gamma_{16}+s_{2} \gamma_{27}=0\left(\right.$ since $\left.B_{45}=0\right)$.

Now let

$$
t:=\left(\gamma_{0,0}, \ldots, \gamma_{0,8}, \gamma_{0,9}[\mu], \gamma_{0,10}[\mu], \gamma_{0,11}[\mu]\right)^{T}=(1,0,0,0,0,0,0,0,0,0,0,0)^{T},
$$

and let $V$ denote the Vandermonde matrix $V\left(z_{0}, \ldots, z_{11}\right)$; then $\rho:=\left(\rho_{0}, \ldots, \rho_{11}\right)$ is uniquely determined by the equation $V \rho=t$. This system is parameterized by a choice of $\alpha, 1<\alpha<\frac{9}{8}$. We are unable to solve the system explicitly in terms of $\alpha$ due to the overwhelming complexity of the algebraic expressions present in $V$. Even in a numerical example, special care is needed because $V$ appears to be poorly conditioned as $\alpha \rightarrow \frac{9}{8}$. We conclude with a Mathematica calculation for $\alpha=1.05$. We compute $\beta \cong 1.98166, \delta \cong 18.4624, u \cong 16.4867, r_{1} \cong 17.6363$ and $r_{2} \cong 0.996995$. The support of $\mu$, calculated using the above formulas, is given by $z_{0} \cong 0.998496 i, z_{1}=-z_{0}, z_{2} \cong 4.19956, z_{3}=-z_{2}, z_{4} \cong 2.09976+3.63692 i$, $z_{5}=-\bar{z}_{4}, z_{6}=\bar{z}_{4}, z_{7}=-z_{4}, z_{8} \cong 0.864723+0.499248 i, z_{9}=-\bar{z}_{8}, z_{10}=\bar{z}_{8}$ and $z_{11}=-z_{8}$. The densities are then computed from the Vandermonde matrix as $\rho_{0} \cong 0.166637, \rho_{2} \cong 0.0000301043, \rho_{1}=\rho_{8}=\rho_{9}=\rho_{10}=\rho_{11}=\rho_{0}, \rho_{3}=\rho_{4}=\rho_{5}=$ $\rho_{6}=\rho_{7}=\rho_{2}$. Note that all atoms in the circle $x^{2}+y^{2}=r_{1}$ have the same density, and similarly for the atoms in $x^{2}+y^{2}=r_{2}$. 


\section{CHAPTER 5}

\section{J.E. McCarthy's Phenomenon and the Proof of Theorem 1.5}

At the very beginning of our study of TCMP, and motivated by the analogies with THMP, our working conjecture was that to solve TCMP for $\gamma \equiv \gamma^{(2 n)}$ one had to find a flat extension for $M(n)$ [CuF4, 1.1 Main Conjecture]. As evidence began to mount on the substantial differences between TCMP and THMP, the possibility of finding truncated moment sequences $\gamma$ admitting representing measures, but not having rank $M(n)$-representing measures, became more tangible. Through an ingenious counting argument, J.E. McCarthy finally established this [McC]. We reproduce here, with permission, his unpublished result; first, we need some notation.

Let $X$ be a nonempty finite set, let $\mathcal{M}(X)$ be the Banach space of signed real measures on $X$, and let $\mathcal{P}(X)$ be the positive cone of measures which assign positive mass to every point of $X$. As a subset of $\mathcal{M}(X), \mathcal{P}(X)$ is open and nonempty. For $f$ a real-valued function on $X$, let $L_{f}: \mathcal{M}(X) \rightarrow \mathbb{R}$ be given by $\mu \mapsto \int f d \mu$, and for $\mathbf{f} \equiv\left(f_{1}, \ldots, f_{N}\right)$, let $L_{\mathbf{f}}:=\left(L_{f_{1}}, \ldots, L_{f_{N}}\right)$.

Lemma 5.1. Let $X \subseteq \mathbb{R}^{2}$ be a finite set and let $f_{1}, \ldots, f_{N}$ be $N$ linearly independent real-valued functions on $X$. Then $L_{\mathbf{f}}(\mathcal{P}(X))$ has nonempty interior in $\mathbb{R}^{N}$.

Proof. We first claim that the map $L_{\mathbf{f}}$ is onto. Since $\left\{f_{1}, \ldots, f_{N}\right\}$ is linearly independent on $X \equiv\left\{x_{1}, \ldots, x_{p}\right\}$, the column rank of the matrix $\mathbf{f}(X):=$ $\left(f_{j}\left(x_{i}\right)\right)_{1 \leq i \leq p, 1 \leq j \leq N}$ is $N$. Then the row rank of $\mathbf{f}(X)$ must also be $N$, so there exists a subset $\mathbf{x} \equiv\left\{x_{p_{1}}, \ldots, x_{p_{N}}\right\} \subseteq X$ such that $\mathbf{f}(\mathbf{x}):=\left(f_{j}\left(x_{p_{i}}\right)\right)_{i, j=1}^{N}$ is invertible. Given a vector $\left(y_{1}, \ldots, y_{N}\right)^{T} \in \mathbb{R}^{N}$, we can then find scalars $\lambda_{1}, \ldots, \lambda_{N} \in \mathbb{R}$ such that

$\left(\begin{array}{c}y_{1} \\ \vdots \\ y_{N}\end{array}\right)=\mathbf{f}(\mathbf{x})\left(\begin{array}{c}\lambda_{1} \\ \vdots \\ \lambda_{N}\end{array}\right)=\lambda_{1}\left(\begin{array}{c}f_{1}\left(x_{p_{1}}\right) \\ \vdots \\ f_{N}\left(x_{p_{1}}\right)\end{array}\right)+\cdots+\lambda_{N}\left(\begin{array}{c}f_{1}\left(x_{p_{N}}\right) \\ \vdots \\ f_{N}\left(x_{p_{N}}\right)\end{array}\right)=L_{\mathbf{f}}\left(\sum_{i=1}^{N} \lambda_{i} \delta_{x_{i}}\right)$,

that is, $\left(y_{1}, \ldots, y_{N}\right)^{T} \in L_{\mathbf{f}}(\mathcal{M}(X))$.

Since $L_{\mathbf{f}}$ is onto, the Open Mapping Theorem guarantees that $L_{\mathbf{f}}$ is open, which implies that $L_{\mathbf{f}}(\mathcal{P}(X))$ is open and nonempty.

Theorem 5.2. $[\mathbf{M c C}]$ There exists a moment sequence $\gamma \equiv \gamma^{(10)}: \gamma_{00}, \ldots$, $\gamma_{0,10}, \ldots, \gamma_{10,0}$ admitting a representing measure, but not admitting a $\operatorname{rank} M(5)$ atomic representing measure.

Proof. Observe first that $\operatorname{rank} M(5) \leq 21$, and that specifying the complex moments $\gamma_{00}, \ldots, \gamma_{0,10}, \ldots, \gamma_{10,0}$ of a proposed rank $M(5)$-atomic representing measure is equivalent to specifying the integrals (over the support of that measure) of 
the 66 real-valued functions $f_{i j}(x, y):=x^{i} y^{j}, 0 \leq i+j \leq 10$ (cf. [CuF4, Subsection $6.2])$. Next, the set $\mathcal{M}_{21}\left(\mathbb{R}^{2}\right)$ of purely atomic real signed measures on the plane, having 21 or fewer atoms, can be put in one-to-one correspondence with $\mathbb{R}^{63}$ via the map $\mu \equiv \sum_{i=0}^{20} \rho_{i} \delta_{\left(x_{i}, y_{i}\right)} \mapsto\left(\rho_{0}, x_{0}, y_{0}, \ldots, \rho_{20}, x_{20}, y_{20}\right)$. Let $\mathbf{f} \equiv\left(f_{i j}\right)_{0 \leq i+j \leq 10}$; the map $\mu \mapsto L_{\mathbf{f}}(\mu)$ can thus be regarded as a polynomial mapping from $\mathbb{R}^{63}$ to $\mathbb{R}^{66}$, whose range $L_{\mathbf{f}}\left(\mathcal{M}_{21}\left(\mathbb{R}^{2}\right)\right)$ must therefore have topological dimension at most 63. On the other hand, the 66 functions $\left\{f_{i j}\right\}_{0 \leq i, j \leq 10}$ are linearly independent on $\mathbb{R}^{2}$, so there must exist a finite subset $X \subseteq \mathbb{R}^{2}$ such that $\left\{f_{i j}\right\}_{0 \leq i, j \leq 10}$ is linearly independent on $X$. By the first part of the proof of Lemma 5.1, we can choose $X$ to have exactly 66 points. For this particular choice of $X$, we can now apply Lemma 5.1 to $\mathbf{g}:=\left\{\left.f_{i j}\right|_{X}\right\}_{0 \leq i, j \leq 10}$ and conclude that $L_{\mathbf{g}}(\mathcal{P})\left(\subseteq L_{\mathbf{g}}(\mathcal{M}(X))\right)$ has topological dimension 66 (by virtue of being a nonempty open set). This shows that $L_{\mathbf{g}}(\mathcal{P}) \nsubseteq L_{\mathbf{f}}\left(\mathcal{M}_{21}\left(\mathbb{R}^{2}\right)\right)$, so there exist sequences in $L_{\mathbf{g}}(\mathcal{P})$ which cannot be represented with positive measures of 21 or fewer atoms.

REMARK 5.3. Observe that for a given $n$, the number of real moments derived from $\gamma^{(2 n)}$ is $(n+1)(2 n+1)$, while rank $M(n) \leq \frac{(n+1)(n+2)}{2}$. Thus, $n=5$ produces the first instance in which 3 times the rank of $M(n)$ (i.e., the number of real "degrees of freedom" allowed by a rank $M(n)$-atomic representing measure in the plane) is less than the number of real moments associated with the given $\gamma$. Thus, in a certain sense, $n=5$ is extremal. Whether for TCMP with $n \leq 4$ the existence of a representing measure implies the existence of a rank $M(n)$-atomic representing measure is an open problem. We know that the answer is affirmative for $n=1$ ([CuF4, Theorem 6.1]), and we have partial affirmative results for $n=2$ (Chapter 3 ) and $n=3,4$ (Chapter 2), but we have no definite conclusion in general.

McCarthy's result might suggest that no invertible $M(5)$ can admit a 21-atomic representing measure. Our next example shows that there do exist sequences $\gamma^{(10)}$ with $M(5)$ invertible and having 21-atomic representing measures.

EXAMPLE 5.4. The following 21-atomic measure gives rise to an invertible $M(5)$. We have obtained the atoms and densities by a simulation using Mathematica's Random. On our machine with $40 \mathrm{MB}$ of RAM, the Mathematica calculation of $\operatorname{det} M(5)$ did not terminate. To establish the invertibility of $M(5)$ we instead used Choleski's Algorithm, first establishing that $\operatorname{det} M(3)>0$, and then checking that $\operatorname{det}\left(C-B^{*} M(3)^{-1} B\right)>0$, where $M(5)=\left(\begin{array}{cc}M(3) & B \\ B^{*} & C\end{array}\right)$.

(On the list below, atoms are labeled $z_{i}$, densities are labeled $\rho_{i}$.)

$$
\begin{array}{rrrrrr}
z_{1}=i / 3 & \rho_{1}=3 & z_{2}=-i / 2 & \rho_{2}=2 & z_{3}=1 & \rho_{3}=2 \\
z_{4}=2 / 3+3 i / 2 & \rho_{4}=3 & z_{5}=-1 & \rho_{5}=1 & z_{6}=i & \rho_{6}=3 \\
z_{7}=1 / 2+i / 3 & \rho_{7}=2 & z_{8}=3 i / 4 & \rho_{8}=2 & z_{9}=2+2 i / 3 & \rho_{9}=2 \\
z_{10}=1+i & \rho_{10}=2 & z_{11}=2 / 3+i / 3 & \rho_{11}=1 & z_{12}=2+3 i / 2 & \rho_{12}=2 \\
z_{13}=3 / 4+i & \rho_{13}=2 & z_{14}=1+i / 2 & \rho_{14}=2 & z_{15}=1 / 3+i / 4 & \rho_{15}=2 \\
z_{16}=1 / 4+i / 3 & \rho_{16}=2 & z_{17}=3 / 2+i / 2 & \rho_{17}=3 & z_{18}=1 / 4 & \rho_{18}=2 \\
z_{19}=-i & \rho_{19}=3 & z_{20}=-1+i / 2 & \rho_{20}=2 & z_{21}=1+2 i / 3 & \rho_{21}=2
\end{array}
$$


We now turn to the proof of Theorem 1.5. Let $K$ denote a closed subset of $\mathbb{R}^{2}$. Given $n>0$, let

$$
\beta_{i j}=\iint_{K} x^{i} y^{j} d x d y \quad(0 \leq i+j \leq n) .
$$

The (real) 2-dimensional quadrature problem ([DaR], $[\mathbf{R e z 1}],[\mathbf{S t r}])$ entails finding nodes $\left(x_{1}, y_{1}\right), \ldots,\left(x_{m}, y_{m}\right) \in K$ and nonnegative weights $a_{1}, \ldots, a_{m}$ such that

$$
\beta_{i j}=\sum_{\ell=1}^{m} a_{\ell} x_{\ell}^{i} y_{\ell}^{j} \quad(0 \leq i+j \leq n) ;
$$

if such a representation exists, one also seeks to find a representation for which $m$ is

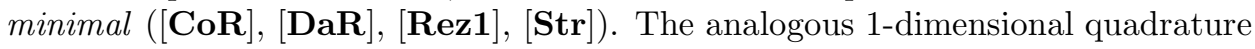
problem with $K=[a, b] \subseteq R$ was solved by C.F. Gauss, who established a minimal quadrature rule with $n$ nodes to interpolate the $2 n-1$ moments $\int_{a}^{b} x^{i} d x(0 \leq i \leq$ $2(n-1))$ (Gaussian Quadrature [Ral $]$ ).

Relatively few results are known concerning minimal rules for (5.1), but the following fundamental result of $\mathrm{V}$. Tchakaloff [Tch] does establish the existence of quadrature rules as in (5.2) for $K$ compact.

THEOREM 5.5. [Tch] Let $K$ be a compact subset of $\mathbb{R}^{2}$ with positive planar measure. Then there exist $N \leq \frac{(n+1)(n+2)}{2}$ nodes $\left(x_{1}, y_{1}\right), \ldots,\left(x_{N}, y_{N}\right) \in K$ and nonnegative weights $a_{1}, \ldots, a_{N}$ such that

$$
\iint_{K} p(x, y) d x d y=\sum_{\ell=1}^{N} a_{\ell} p\left(x_{\ell}, y_{\ell}\right)
$$

for every $p \in \mathbb{R}[x, y]$ with $\operatorname{deg} p \leq n$.

Tchakaloff also proved that in some cases $N=\frac{(n+1)(n+2)}{2}$ is minimal, and he also formulated corresponding results for quadrature problems in $\mathbb{R}^{d}$ with $d>2$. Using the correspondence between the truncated real moment problem for $\mathbb{R}^{2 n}$ and TCMP for $\mathbb{C}^{n}[\mathbf{C u F 4}$, Section 7], it is straightforward to reformulate Tchakaloff's result as follows. In the sequel, we say that a linear functional $F: \mathcal{P}_{n} \rightarrow \mathbb{C}$ is $K$-positive if

$$
p \in \mathcal{P}_{n},\left.p\right|_{K} \geq 0 \Longrightarrow F(p) \geq 0 \text {. }
$$

Proposition 5.6. Let $K$ be a compact subset of $\mathbb{C}$. If $F: \mathcal{P}_{n} \rightarrow \mathbb{C}$ is $K$ positive, then there exist $N \leq m(n)$ points $z_{1}, \ldots, z_{N} \in K$ and nonnegative numbers $a_{1}, \ldots, a_{N}$ such that $F(p)=\sum_{\ell=1}^{N} a_{\ell} p\left(z_{\ell}, \bar{z}_{\ell}\right), p \in \mathcal{P}_{n}$.

As an immediate consequence of Proposition 5.6 we can prove Theorem 1.5 (iii) $\Rightarrow$ (iv).

Proposition 5.7. Suppose $\gamma \equiv \gamma^{(2 n)}$ has a representing measure with compact support $K$. Then there exist $M \leq m(2 n+2) \equiv(n+2)(2 n+3)$ points $z_{1}, \ldots, z_{M} \in K$, and nonnegative weights $a_{1}, \ldots, a_{M}$ such that $\gamma_{i j}=\sum_{\ell=1}^{M} a_{\ell} \bar{z}_{\ell}^{i} z_{\ell}^{j}(0 \leq i+j \leq 2 n)$.

Proof. Let $\mu$ denote a representing measure such that $K \equiv \operatorname{supp} \mu$ is compact. Apply Proposition 5.6 to the functional $F: \mathcal{P}_{2 n} \rightarrow \mathbb{C}$ defined by $F(p):=\int p d \mu$. 
In [Mys] I.P. Mysovskikh generalized Tchakaloff's Theorem to the case where $K \subseteq \mathbb{R}^{2}$ is closed but possibly unbounded, and where $d x d y$ is replaced by $\rho(x, y) d x d y$ for a nonnegative weight function $\rho$; moreover, the results of [Mys] are valid for quadrature problems in $\mathbb{R}^{d}$ with $d>2$. Recently, in response to our question, M. Putinar [Pu7] extended Tchakaloff's Theorem further, to the case of unbounded closed $K \subseteq \mathbb{R}^{d}$ and moments of arbitrary positive Borel measures. Putinar's results have the following consequences for TCMP.

Proposition 5.8. (cf. [Pu7]) If $\gamma \equiv \gamma^{(2 n)}$ has a representing measure $\mu$ such that $\mathcal{P}_{2 n+2} \subseteq L^{1}(\mu)$, then there exist $M \leq m(2 n+2) \equiv(n+2)(2 n+3)$ points $z_{1}, \ldots, z_{M} \in \operatorname{supp} \mu$ and positive numbers $a_{1}, \ldots, a_{M}$ such that

$$
\int p d \mu=\sum_{\ell=1}^{M} a_{\ell} p\left(z_{\ell}, \bar{z}_{\ell}\right) \quad\left(p \in \mathcal{P}_{2 n+1}\right)
$$

in particular $\gamma_{i j}=\sum_{\ell=1}^{M} a_{\ell} \bar{z}_{\ell}^{i} z_{\ell}^{j}(0 \leq i+j \leq 2 n)$.

Proposition 5.8 immediately yields Theorem $1.5(\mathrm{i}) \Rightarrow(\mathrm{iv})$; thus, in Theorem 1.5 we already have the equivalence of (i), (ii), (iii) and (iv). We next prove (iv) $\Leftrightarrow(\mathrm{v})$.

Proposition 5.9. $\gamma \equiv \gamma^{(2 n)}$ has a finitely atomic representing measure if and only if there exists $k \geq 0$ such that $M(n)$ admits a positive extension $M(n+k)$ which in turn admits a flat extension $M(n+k+1)$.

Proof. Suppose $M(n+k) \geq 0$ has a flat extension $M(n+k+1)$. Theorem 1.1 implies that the $M(n+k)$ moment problem has a rank $M(n+k)$-atomic representing measure; this is also a finitely atomic representing measure for $\gamma$.

Conversely, suppose $\gamma$ has an $r$-atomic representing measure $\mu$; then

$$
M(n) \geq 0, \mathcal{P}_{n} \subseteq L^{2}(\mu) \text {, and }\left\{1, z, \ldots, z^{r-1}\right\} \text { is a basis for } L^{2}(\mu)
$$

[CuF4, Lemma 3.6].

Two cases arise.

Case 1: $r \leq n$. Here (5.3) and (1.4) imply that $\left\{1, Z, \ldots, Z^{r-1}\right\}$ is a basis for $\mathcal{C}_{M(n)}$. Thus $M(n) \geq 0$ is flat, and has a flat extension $M(n+1)$ by Theorem 1.4.

Case 2: $r>n$. Consider $M(r)[\mu]$, which is a positive extension of $M(n)$. From (5.3) and (1.4), it follows that $\left\{1, Z, \ldots, Z^{r-1}\right\}$ spans $\mathcal{C}_{M(r)}$, so $M(r)[\mu]$ is flat and thus has a flat extension $M(r+1)$.

In Theorem 1.5, the estimates on the number of atoms (iv) and the size of $k$ (v) follow immediately from Proposition 5.8 and the preceding argument.

To complete the proof of Theorem 1.5, we next prove (iv) $\Leftrightarrow(v i)$; this yields an operator-theoretic criterion for the existence of finitely-atomic representing measures for $\gamma^{(2 n)}$. Suppose $M(n)(\gamma) \geq 0$; then $\langle\cdot, \cdot\rangle_{M(n)(\gamma)}$ defines a semi-inner product on $\mathcal{P}_{n}$ which we abbreviate by $\langle\cdot, \cdot\rangle_{\gamma}$, i.e., $\langle f, g\rangle_{\gamma}:=(M(n)(\gamma) \hat{f}, \hat{g})$ $\left(f, g \in \mathcal{P}_{n}\right)$. Let $\mathcal{N}:=\left\{f \in \mathcal{P}_{n}:\langle f, f\rangle_{\gamma}=0\right\}$ and note that $\mathcal{N}=\operatorname{ker} M(n)$. Indeed, for $f \in \mathcal{N}, 0=\langle f, f\rangle_{\gamma}=(M(n)(\gamma) \hat{f}, \hat{f})=\left\|(M(n)(\gamma))^{1 / 2} \hat{f}\right\|^{2}$; thus $(M(n)(\gamma))^{1 / 2} \hat{f}=0$ whence $M(n)(\gamma) \hat{f}=0$.

Proposition 5.10. $\gamma \equiv \gamma^{(2 n)}$ has a finitely atomic representing measure if and only if $M(n)(\gamma) \geq 0$ and $\langle\cdot, \cdot\rangle_{\gamma}$ extends to a semi-inner product $\langle\cdot, \cdot\rangle$ on $\mathbb{C}[z, \bar{z}]$ such that 
(i) $\mathcal{M}:=\left\{f \in \mathbb{C}[z, \bar{z}]:\langle f, f\rangle_{\gamma}=0\right\}$ is an ideal in $\mathbb{C}[z, \bar{z}]$;

(ii) $\mathcal{H}:=\mathbb{C}[z, \bar{z}] / \mathcal{M}$, with the inner product induced by $\langle\cdot, \cdot\rangle_{\gamma}$, is a finitedimensional Hilbert space; and

(iii) the operator $M_{z}: \mathcal{H} \rightarrow \mathcal{H}$ defined by $M_{z}([f]):=[z f](f \in \mathbb{C}[z, \bar{z}])$ is normal.

Proof. Suppose the desired extension exists; it then follows exactly as in the proof of [CuF4, Theorem 4.7] that $\gamma$ has a representing measure $\mu$ with supp $\mu \subseteq$ $\sigma\left(M_{z}\right)$ (the spectrum of $M_{z}$ ); thus card $\operatorname{supp} \mu \leq \operatorname{card} \sigma\left(M_{z}\right) \leq \operatorname{dim} \mathcal{H}<\infty$.

Conversely, suppose $\gamma$ has a finitely atomic representing measure. It follows from Proposition 5.9 that there exists $k \geq 0$ such that $M(n)$ admits a positive extension $M(n+k)$, which in turn has a flat extension $M(n+k+1)$. Thus $[\mathbf{C u F} 4$, Corollary 5.12] implies that there exists a unique positive flat moment matrix extension $M(\infty)$ of $M(n+k)$; we can then let $\langle f, g\rangle:=(M(\infty) \hat{f}, \hat{g})$. Exactly as in the proof of [CuF4, Theorem 4.7], it now follows that $\langle\cdot, \cdot\rangle$ satisfies (i)-(iii).

Added in Proof. In forthcoming papers [CuF6] [Fia2] we apply the extension theory of moment matrices to the truncated $K$-moment problem and the multivariable quadrature problem. Given $M(n) \equiv M(n)(\gamma), k \leq n$, and a polynomial $p(z, \bar{z})$ of degree $2 k$ or $2 k-1$, there exists a unique matrix $M_{p}(n) \equiv M_{p}(n)(\gamma)$ (of size $\left.\frac{(n-k+1)(n-k+2)}{2}\right)$ such that

$$
\left(M_{p}(n) \hat{f}, \hat{g}\right)=\Lambda(p f \bar{g}) \quad\left(f, g \in \mathcal{P}_{n-k}\right),
$$

where $\Lambda$ is the Riesz functional on $\mathcal{P}_{2 n}$ defined by $\Lambda\left(\sum a_{i j} \bar{z}^{i} z^{j}\right):=\sum a_{i j} \gamma_{i j} . M_{p}(n)$ may be computed as a certain linear combination of compressions of $M(n)$. The following result characterizes the existence of a minimal representing measure supported in a prescribed semi-algebraic set.

Let $\mathcal{P} \equiv\left\{p_{1}, \ldots, p_{m}\right\}$, with $p_{i} \in \mathbb{C}[z, \bar{z}]$, let $k_{i}:=\operatorname{deg} p_{i}$, and let $K_{\mathcal{P}}:=\{z$ : $\left.p_{i}(z, \bar{z}) \geq 0(1 \leq i \leq m)\right\}$. Recall from [CuF4] that if $M(n) \geq 0$ admits a flat extension $M(n+1)$, then $M(n+1)$ admits unique successive flat (positive) extensions $M(n+2), M(n+3), \ldots$

Theorem. [CuF6] There exists a rank $M(n)$-atomic (minimal) representing measure for $\gamma^{(2 n)}$ supported in $K_{\mathcal{P}}$ if and only if $M(n)$ admits a flat extension $M(n+1)$ for which $M_{p_{i}}\left(n+k_{i}\right) \geq 0(1 \leq i \leq m)$.

[CuF6] also contains a thorough analysis of the quadratic moment problem for the disk. In $[\mathbf{F i a 2}]$ the preceding theorem and $[\mathbf{C u F} 4]$ are used to develop new minimal quadrature rules for the disk, square, triangle, and annulus. 


\section{APPENDIX}

\section{Summary of Results}

Notations and AbBreviations. In all cases, the underlying moment matrix $M(n)$ is assumed to be positive and recursively generated. Results are seminumerical or numerical unless designated General $(\mathrm{G})$, in which case the result includes the most general (positive, recursively generated) moment matrix with the indicated relations and/or properties.

$$
\begin{array}{ll}
r: & \operatorname{rank} M(n) . \\
c: & \operatorname{card} \mathcal{V}(\gamma) . \\
\rho: & \rho(\gamma) .
\end{array}
$$

Rel/Prop: Column relations and/or additional properties satisfied by $M(n)$.

MMEB: Does $M(n)$ admit a moment matrix extension block $B$ such that $\operatorname{Ran} B \subseteq \operatorname{Ran} M(n)$ ? Y and N stand for Yes and No, respectively; the label $\mathrm{T}$ indicates that Theorem 2.7 could be used to obtain a moment matrix extension block $B$, even though we may have used some other result in the reference cited.

$C$-Test: What equations are required for the reduced $C$-test?

FE: How many flat extensions does $M(n)$ have?

FRM: How many finitely atomic representing measures does $M(n)$ admit?

Ref: Reference to the result listed.

Entries for which we do not have information have been left blank.

\begin{tabular}{|c|c|c|c|c|c|c|c|c|}
\hline & & & & & $n=1$ & & & \\
\hline$r$ & $c$ & $\rho$ & Rel/Prop & MMEB & $C$-Test & $\mathrm{FE}$ & FRM & Ref \\
\hline 1 & 1 & 0 & $\begin{array}{c}(\mathrm{G}) \\
Z=\alpha 1\end{array}$ & $\mathrm{YT}$ & $\varnothing$ & 1 & 1 & [CuF4, Theorem 6.1] \\
\hline 2 & $\infty$ & $\infty$ & $\bar{Z}=\alpha 1+\beta Z$ & $\mathrm{Y}$ & $C_{11}=C_{22}$ & $\infty$ & $\infty$ & [CuF4, Theorem 6.1] \\
\hline 3 & $\infty$ & $\infty$ & $(\mathrm{G})$ & $\mathrm{Y}$ & $C_{11}=C_{22}$ & $\infty$ & $\infty$ & [CuF4, Theorem 6.1] \\
\hline
\end{tabular}

Under $C$-Test, NA stands for "not applicable" (this appears when the corresponding entry under MMEB is $\mathrm{N}$ ). 


\begin{tabular}{|c|c|c|c|c|c|c|c|c|}
\hline & & & & $n=2$ & & & & \\
\hline$r$ & $c$ & $\rho$ & Rel/Prop & MMEB & $C$-Test & $\mathrm{FE}$ & FRM & Ref \\
\hline 4 & 4 & 0 & $Z^{2}=\alpha Z+\beta \bar{Z}$ & $\bar{Y}$ & $\varnothing$ & 1 & 1 & $\begin{array}{c}\text { CuF4, } \\
\text { Example } \\
3.3]\end{array}$ \\
\hline 4 & 4 & 0 & $\begin{array}{c}(\mathrm{G}) \\
\bar{Z} Z=\alpha+\bar{\beta} Z+\beta \bar{Z} \\
\text { real moments } \\
\left\{1, Z, \bar{Z}, Z^{2}\right\} \text { basis }\end{array}$ & $\mathrm{Y}$ & $C_{11}=C_{22}$ & 1 & 1 & $\begin{array}{c}\text { Theorem } \\
3.7\end{array}$ \\
\hline 5 & $\infty$ & $\infty$ & $\begin{array}{c}(\mathrm{G}) \\
\bar{Z} Z=\alpha+\bar{\beta} Z+\beta \bar{Z}\end{array}$ & $\mathrm{Y}(\mathrm{Th} 2.7)$ & $C_{11}=C_{22}$ & 1 & 1 & $\begin{array}{l}\text { Example } \\
2.16\end{array}$ \\
\hline 5 & $\infty$ & $\infty$ & $\bar{Z} Z=\alpha+\bar{\beta} Z+\beta \bar{Z}$ & $\mathrm{Y}(\mathrm{Th} 2.7)$ & $C_{11}=C_{22}$ & $\infty$ & $\infty$ & $\begin{array}{c}\text { Example } \\
3.8\end{array}$ \\
\hline 5 & $\infty$ & $\infty$ & $\bar{Z} Z=\alpha+\bar{\beta} Z+\beta \bar{Z}$ & $\mathrm{Y}(\mathrm{Th} 2.7)$ & $C_{11}=C_{22}$ & $\infty$ & $\infty$ & $\begin{array}{c}\text { Proposition } \\
3.9\end{array}$ \\
\hline
\end{tabular}

\begin{tabular}{|c|c|c|c|c|c|c|c|c|}
\hline & & & & $n=3$ & & & & \\
\hline$r$ & $c$ & $\rho$ & Rel/Prop & MMEB & $C$-Test & $\mathrm{FE}$ & FRM & Ref \\
\hline 7 & 7 & 0 & $\begin{array}{c}\bar{Z}^{3}=Z^{3} \\
\bar{Z} Z^{2} \in \mathcal{P}_{2} \\
\left.\ldots, \bar{Z}^{2}, Z^{3}\right\} \text { basis }\end{array}$ & YT & $C_{11}=C_{22}$ & 1 & 1 & $\begin{array}{c}\text { Example } \\
4.9\end{array}$ \\
\hline 8 & & & $\begin{array}{c}(\mathrm{G}) \\
Z^{3} \in \mathcal{P}_{2}\end{array}$ & YT & $C_{22}=C_{33}$ & & & $\begin{array}{c}\text { Example } \\
2.1\end{array}$ \\
\hline 8 & 2 & -6 & $\begin{array}{c}\text { special case of } \\
Z^{3} \in \mathcal{P}_{2}\end{array}$ & YT & $C_{22}=C_{33}$ & None & None & $\begin{array}{c}\text { Examples } \\
2.14,4.4\end{array}$ \\
\hline 8 & & & $\begin{array}{c}(\mathrm{G}) \\
\bar{Z} Z^{2} \in \mathcal{P}_{2}\end{array}$ & YT & $C_{11}=C_{22}$ & & & $\begin{array}{c}\text { Example } \\
2.15\end{array}$ \\
\hline 8 & $\infty$ & $\infty$ & $\begin{array}{c}\text { special case of } \\
\bar{Z} Z^{2} \in \mathcal{P}_{2}\end{array}$ & YT & $C_{11}=C_{22}$ & $\infty$ & $\infty$ & $\begin{array}{c}\text { Example } \\
4.8\end{array}$ \\
\hline 10 & $\infty$ & $\infty$ & $M(3)>0$ & Y & $\begin{array}{l}C_{11}=C_{22} \\
C_{21}=C_{32}\end{array}$ & None & None & $\begin{array}{c}{[\mathbf{C u F 5},} \\
\text { Section 4] }\end{array}$ \\
\hline
\end{tabular}




\begin{tabular}{|c|c|c|c|c|c|c|c|c|}
\hline & & & & & $n=4$ & & & \\
\hline$r$ & $c$ & $\rho$ & Rel/Prop & MMEB & $C$-Test & $\mathrm{FE}$ & FRM & Ref \\
\hline 115 & $\leq r$ & $\leq 13$ & $\begin{array}{c}\left\{1, \ldots, \bar{Z}^{3}, Z^{4}\right\} \\
\quad \text { l.i. } \\
\bar{Z} Z^{3}, \bar{Z}^{3} Z \in \mathcal{P}_{3}\end{array}$ & $\begin{array}{l}3 \\
3\end{array}$ & NA & $\begin{array}{c}\text { None } \\
\text { (No NC) }\end{array}$ & None & $\begin{array}{c}\text { Examples } \\
2.2,2.5 \\
4.6\end{array}$ \\
\hline 11 & 0 & -11 & $\begin{array}{l}\text { the preceding } \\
\text { with } \\
\bar{Z}^{2} Z^{2} \in \mathcal{P}_{3}\end{array}$ & $\mathrm{~N}$ & NA & $\begin{array}{c}\text { None } \\
\text { (No RC) }\end{array}$ & None & $\begin{array}{c}\text { Examples } \\
2.2,4.6\end{array}$ \\
\hline 12 & 12 & 0 & $\begin{array}{c}\bar{Z}^{2} Z^{2} \in \mathcal{P}_{3} \\
\bar{Z}^{3} Z \in\left\langle\bar{Z}^{2}, Z^{4}\right\rangle \\
\bar{Z}^{4} \in\left\langle Z^{2}, \bar{Z} Z^{3}\right\rangle\end{array}$ & $\begin{array}{ll} & \mathrm{Y} \quad \\
\left.{ }^{4}\right\rangle & \end{array}$ & $\begin{array}{c}C_{11}=C_{22}=C_{33} \\
C_{21}=C_{32} \\
C_{31}=C_{42}\end{array}$ & 1 & 1 & $\begin{array}{c}\text { Example } \\
4.10\end{array}$ \\
\hline 13 & 13 & 0 & $\begin{array}{c}\text { special instance } \\
\text { of } 11 \leq r \leq 13 \\
\text { case }\end{array}$ & e $\mathrm{N}$ & NA & $\begin{array}{c}\text { None } \\
(\text { No NC) }\end{array}$ & None & $\begin{array}{c}\text { Examples } \\
2.5,4.7\end{array}$ \\
\hline
\end{tabular}

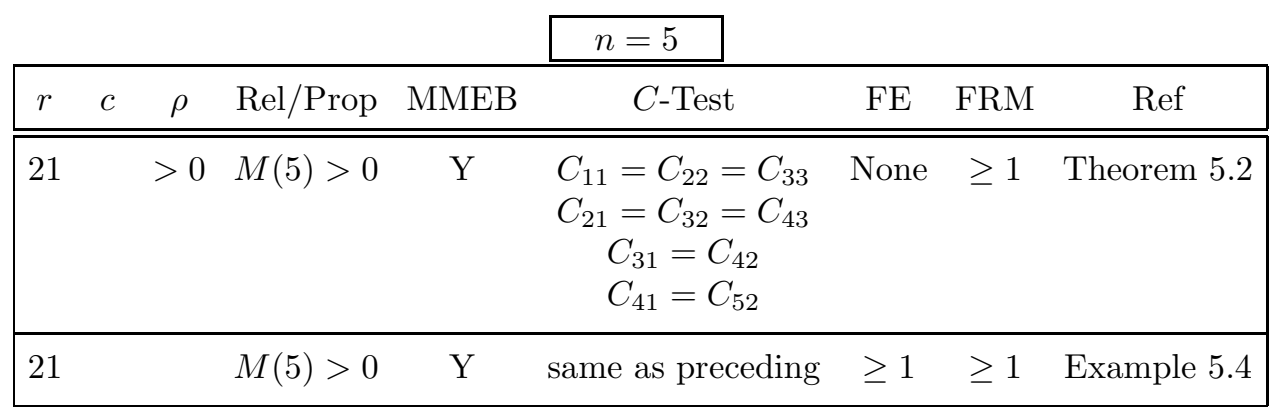

General $n$

\begin{tabular}{|ccccccc|}
\hline Rel/Prop & MMEB & $C$-Test & FE & FRM & Ref \\
\hline \hline $\begin{array}{c}\text { Rank } M(n)=\text { Rank } M(n-1) \\
\text { (Flat data) }\end{array}$ & YT & $\varnothing$ & 1 & 1 & {$[\mathbf{C u F 4}$, Theorem 5.3] } \\
\hline $\bar{Z}=\alpha 1+\beta Z$ & $\mathrm{Y}$ & $\varnothing$ & $\infty$ & $\infty$ & {$[\mathbf{C u F 5}$, Theorem 2.1] } \\
\hline$Z^{k}=p(Z, \bar{Z}), \operatorname{deg} p \leq\left[\frac{n}{2}\right]+1$ & $\mathrm{YT}$ & $\varnothing$ & 1 & $\geq 1$ & {$[\mathbf{C u F 5}$, Theorem 3.1] } \\
\hline $\begin{array}{c}\left\{1, Z, Z^{2}, \ldots, Z^{n}\right\} \operatorname{spans} \mathcal{C}_{M(n)} \\
M(2)>0\end{array}$ & $\mathrm{Y}$ & $\varnothing$ & 1 & 1 & $\begin{array}{c}{[\mathbf{C u F 4},} \\
\text { Corollary 5.16] }\end{array}$ \\
\hline
\end{tabular}




\section{Bibliography}

[AK] N.I. Ahiezer and M.G. Krein, Some Questions in the Theory of Moments, Transl. Math. Monographs vol. 2, Amer. Math. Soc., Providence, 1962.

[Akh] N.I. Akhiezer, The Classical Moment Problem, Hafner Publ. Co., New York, 1965.

[And] T. Ando, Truncated moment problems for operators, Acta Scient. Math. 31 (1970), 319333.

[Atz] A. Atzmon, A moment problem for positive measures on the unit disc, Pacific J. Math. 59 (1975), 317-325.

[Ber] C. Berg, The multidimensional moment problem and semigroups, Moments in Mathematics, Proc. Symposia Appl. Math. vol. 37, Amer. Math. Soc., Providence, 1987, pp. 110124.

[BCJ] C. Berg, J.P.R. Christensen and C.U. Jensen, A remark on the multidimensional moment problem, Math. Ann. 223 (1979), 163-169.

[BeM] C. Berg and P.H. Maserick, Polynomially positive definite sequences, Math. Ann. 259 (1982), 487-495.

[Cas] G. Cassier, Problème des moments sur un compact de $\mathbf{R}^{n}$ et décomposition des polynômes à plusieurs variables, J. Funct. Anal. 58 (1984), 254-266.

[Con] J. Conway, Subnormal Operators, Pitman Publ. Co., London, 1981.

[CoR] R. Cools and P. Rabinowitz, Monomial cubature rules since "Stroud": a compilation, J. Comp. Appl. Math. 48 (1993), 309-326.

[Cu1] R. Curto, Quadratically hyponormal weighted shifts, Integral Eq. Operator Th. 13 (1990), 49-66.

[Cu2] R. Curto, Joint hyponormality: A bridge between hyponormality and subnormality, Proc. Symp. Pure Math., vol. 51, Part 2, Amer. Math. Soc., Providence, 1990, pp. 69-91.

[Cu3] R. Curto, Polynomially hyponormal operators on Hilbert space, Rev. Unión Mat. Arg. 37 (1991), 29-56.

[CuF1] R. Curto and L. Fialkow, Recursively generated weighted shifts and the subnormal completion problem, Integral Equations and Operator Theory 17 (1993), 202-246.

[CuF2] R. Curto and L. Fialkow, Recursively generated weighted shifts and the subnormal completion problem, II, Integral Equations and Operator Theory 18 (1994), 369-426.

[CuF3] R. Curto and L. Fialkow, Recursiveness, positivity, and truncated moment problems, Houston J. Math. 17 (1991), 603-635.

[CuF4] R. Curto and L. Fialkow, Solution of the truncated complex moment problem for flat data, Memoirs Amer. Math. Soc. no. 568, Amer. Math. Soc., Providence, 1996.

[CuF5] R. Curto and L. Fialkow, Flat extensions of positive moment matrices: Relations in analytic or conjugate terms, Operator Th.: Adv. Appl., to appear.

[CuF6] R. Curto and L. Fialkow, The truncated K-moment problem, in preparation.

[CuP1] R. Curto and M. Putinar, Existence of non-subnormal polynomially hyponormal operators, Bull. Amer. Math. Soc. 25 (1991), 373-378.

[CuP2] R. Curto and M. Putinar, Nearly subnormal operators and moment problems, J. Funct. Anal. 115 (1993), 480-497.

[DaR] P.J. Davis and P. Rabinowitz, Methods of Numerical Integration, Academic Press, Inc., Orlando, 1984.

[Dou] R.G. Douglas, On majorization, factorization, and range inclusion of operators on Hilbert spaces, Proc. Amer. Math. Soc. 17 (1966), 413-415.

[Fia1] L. Fialkow, Positivity, extensions and the truncated complex moment problem, Contemporary Math. 185 (1995), 133-150.

[Fia2] L. Fialkow, Multivariable quadrature and extensions of moment matrices, preprint, 1996. 
[Fug] B. Fuglede, The multidimensional moment problem, Expo. Math. 1 (1983), 47-65.

[Hau] F. Hausdorff, Momentprobleme für ein endliches Intervall, Math. Zeit. 16 (1923), 220248.

[Hav1] E.K. Haviland, On the momentum problem for distributions in more than one dimension, Amer. J. Math. 57 (1935), 562-568.

[Hav2] E.K. Haviland, On the momentum problem for distributions in more than one dimension, Part II, Amer. J. Math. 58 (1936), 164-168.

[Hil] D. Hilbert, Über die Darstellung definiter Formen als Summen von Formenquadraten, Math. Ann. 32 (1888), 342-350.

[JeL] N. Jewell and A. Lubin, Commuting weighted shifts and analytic function theory in several variables, J. Operator Th. 1 (1979), 207-223.

[KrN] M.G. Krein and A. Nudel'man, The Markov Moment Problem and Extremal Problems, Transl. Math. Monographs, vol. 50, Amer. Math. Soc., Providence, 1977.

[Lan] H. Landau, Classical background of the moment problem, Moments in Mathematics, Proc. Symposia Appl. Math., vol. 37, Amer. Math. Soc., Providence, 1987, pp. 1-15.

[Li] X. Li, Moment Sequences and Their Applications, Ph.D. dissertation, Virginia Tech. Univ., 1994.

$[\mathrm{McC}] \quad$ J.E. McCarthy, private communication.

$[\mathrm{McCY}]$ J.E. McCarthy and L. Yang, Subnormal operators and quadrature domains, Advances in Mathematics, to appear.

[McG] J.L. McGregor, Solvability criteria for certain N-dimensional moment problems, J. Approx. Theory 30 (1980), 315-333.

[Mys] I.P. Mysovskikh, On Chakalov's Theorem, USSR Comp. Math. 15 (1975), 221-227.

[Nar] F.J. Narcowich, R-operators II: On the approximation of certain operator-valued analytic functions and the Hermitian moment problem, Indiana Univ. Math. J. 26 (1977), 483513.

[Pu1] M. Putinar, A two-dimensional moment problem, J. Funct. Anal. 80 (1988), 1-8.

[Pu2] M. Putinar, The L problem of moments in two dimensions, J. Funct. Anal. 94 (1990), 288-307.

[Pu3] M. Putinar, Positive polynomials on compact semi-algebraic sets, Indiana Univ. Math. J. 42 (1993), 969-984.

[Pu4] M. Putinar, Extremal solutions of the two-dimensional L-problem of moments, J. Funct. Anal. 136 (1996), 331-364.

[Pu5] M. Putinar, Extremal solutions of the two-dimensional L-problem of moments, II, MSRI Preprint 105-95.

[Pu6] M. Putinar, Linear analysis of quadrature domains, Ark. för Mat. 33 (1995), 357-376.

[Pu7] M. Putinar, On Tchakaloff's Theorem, preprint 1995.

[Ral] A. Ralston, A first Course in Numerical Analysis, McGraw-Hill, New York, 1965.

[Rez1] B. Reznick, Sums of Even Powers of Real Linear Forms, Memoirs Amer. Math. Soc., vol. 96, no. 463, Amer. Math. Soc., Providence, 1992.

[Rez2] B. Reznick, private communication.

[Sar] D. Sarason, Moment problems and operators on Hilbert space, Moments in Mathematics, Proc. Symposia Appl. Math., vol. 37, Amer. Math. Soc., Providence, 1987, pp. 54-70.

[Sch1] K. Schmüdgen, An example of a positive polynomial which is not a sum of squares of polynomials. A positive, but not strongly positive functional, Math. Nachr. 88 (1979), 385-390.

[Sch2] K. Schmüdgen, The K-moment problem for semi-algebraic sets, Math. Ann. 289 (1991), 203-206.

[SeS] Z. Sebestyén and J. Stochel, Restrictions of positive self-adjoint operators, Acta Scient. Math. 55 (1991), 149-154.

[ShT] J. Shohat and J. Tamarkin, The Problem of Moments, Math. Surveys I, Amer. Math. Soc., Providence, 1943.

[Smu] J.L. Smul'jan, An operator Hellinger integral (Russian), Mat. Sb. 91 (1959), 381-430.

[Sto] J. Stochel, private communication.

[StSz1] J. Stochel and F. Szafraniec, A characterization of subnormal operators, Spectral theory of linear operators and related topics, Birkhäuser (1984), pp. 261-263.

[StSz2] J. Stochel and F. Szafraniec, Unbounded weighted shifts and subnormality, Integral Eq. Operator Th. 12 (1989), 146-153. 
[StSz3] J. Stochel and F. Szafraniec, On normal extensions of unbounded operators, III: Spectral properties, Publ. RIMS 25 (1989), 105-139.

[StSz4] J. Stochel and F. Szafraniec, Algebraic operators and moments on algebraic sets, Portugaliae Math. 51 (1994), 1-21.

[Str] A.H. Stroud, Approximate Calculation of Multiple Integrals, Prentice-Hall, 1971.

[Sza1] F. Szafraniec, Boundedness of the shift operator related to positive definite forms: An application to moment problems, Ark. Mat. 19 (1981), 251-259.

[Sza2] F. Szafraniec, Moments on compact sets, Prediction Theory and Harmonic Analysis (V. Mandrekar and H. Salehi, eds.), North-Holland, Amsterdam, 1983, pp. 379-385.

[Tch] V. Tchakaloff, Formules de cubatures mécaniques à coefficients non négatifs, Bull. Sc. Math. 81 (1957), 123-134.

[Wol] Wolfram Research, Inc., Mathematica, Version 2.1, Wolfram Research, Inc., Champaign, Illinois, 1992. 


\section{List of Symbols}

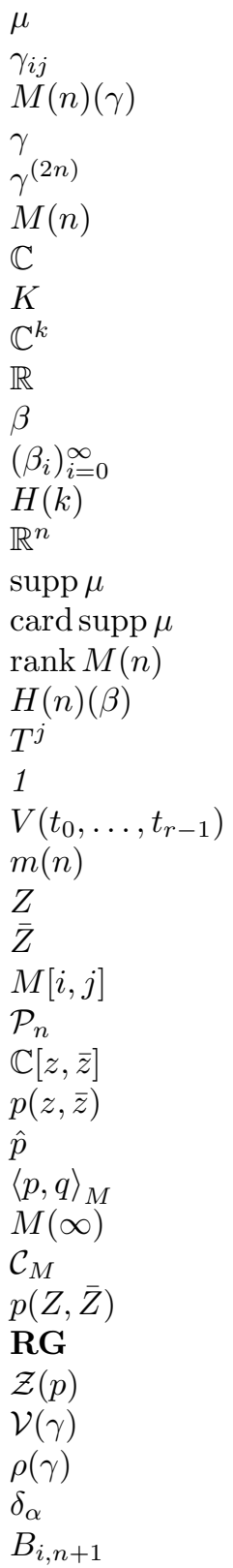

\begin{tabular}{r|lr} 
ii & $\operatorname{Ran} B$ & 8 \\
ii & {$[M(n) ; B]$} & 8 \\
ii & {$\left[\frac{n}{2}\right]$} & 8 \\
ii & $\operatorname{deg} p$ & 9 \\
ii & $\tilde{A}$ & 11 \\
ii & $\tilde{\gamma}$ & 12 \\
1 & $\alpha$ & 13 \\
1 & $\delta$ & 13 \\
1 & $\triangle$ & 13 \\
2 & $\mathbf{v}$ & 14 \\
13 & $\mathcal{R}(k, \ell)$ & 14 \\
2 & {$[\mathbf{w}]_{(d)}$} & 14 \\
2 & $\mathbf{R C}$ & 15 \\
2 & $\mathcal{T}(i, j, p)$ & 15 \\
2 & $\mathcal{T}$ & 15 \\
2 & $\mathcal{T}^{\prime}$ & 15 \\
2 & $\mathbf{N C}$ & 16 \\
3 & $I$ & 17 \\
3 & $\left\{B_{\alpha}\right\}_{\alpha \in A}$ & 18 \\
3 & $\mathcal{R}$ & 18 \\
3 & $\mathcal{S}$ & 18 \\
4 & $\mathcal{S}^{\prime}$ & 18 \\
4 & $M_{I}$ & 18 \\
4 & $V$ & 18 \\
4 & $L$ & 20 \\
4 & $L^{\prime}$ & 20 \\
4 & $M^{\prime}$ & 20 \\
4 & $h$ & 20 \\
4 & $P$ & 25 \\
4 & {$[A]_{\left\{n_{1}, \ldots, n_{k}\right\}}$} & 29 \\
4 & {$[M(3)]_{\text {anal }}$} & 31 \\
5 & $a_{r}$ & 31 \\
5 & $a_{s}$ & 31 \\
6 & $b_{r}$ & 31 \\
6 & $b_{s}$ & 38 \\
6 & $\mathcal{M}_{I}$ & 44 \\
6 & $\mathcal{P}(X)$ & 44 \\
7 & $L_{f}$ & 44 \\
7 & $\mathbf{f}$ & 44 \\
& &
\end{tabular}


LIST OF SYMBOLS

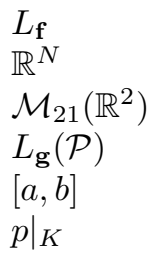

\begin{tabular}{l|ll}
44 & $L^{2}(\mu)$ & 47 \\
44 & $\mathcal{N}$ & 47 \\
45 & $\mathcal{M}$ & 48 \\
45 & $\mathcal{H}$ & 48 \\
46 & $M_{z}$ & 48 \\
46 & $\sigma\left(M_{z}\right)$ & 48
\end{tabular}

